\title{
Search for gamma-ray spectral lines with the Fermi Large Area Telescope and dark matter implications
}

M. Ackermann, ${ }^{1}$ M. Ajello, ${ }^{2}$ A. Albert, ${ }^{3, *}$ A. Allafort, ${ }^{4}$ L. Baldini,${ }^{5}$ G. Barbiellini, ${ }^{6,7}$ D. Bastieri,${ }^{8,9}$ K. Bechtol, ${ }^{4}$ R. Bellazzini, ${ }^{10}$ E. Bissaldi, ${ }^{11}$ E. D. Bloom,${ }^{4,}$ E. Bonamente, ${ }^{12,13}$ E. Bottacini,${ }^{4}$ T. J. Brandt, ${ }^{14}$ J. Bregeon, ${ }^{10}$ M. Brigida, ${ }^{15,16}$ P. Bruel, ${ }^{17}$ R. Buehler, ${ }^{1}$ S. Buson, ${ }^{8,9}$ G. A. Caliandro, ${ }^{18}$ R. A. Cameron, ${ }^{4}$ P. A. Caraveo, ${ }^{19}$ J. M. Casandjian, ${ }^{20}$ C. Cecchi, ${ }^{12,13}$ E. Charles, ${ }^{4, *}$ R. C. G. Chaves, ${ }^{20}$ A. Chekhtman, ${ }^{21}$ J. Chiang, ${ }^{4}$ S. Ciprini,${ }^{22,23}$ R. Claus, ${ }^{4}$ J. Cohen-Tanugi, ${ }^{24}$ J. Conrad, ${ }^{25,26,27}$ S. Cutini, ${ }^{22,23}$ F. D'Ammando, ${ }^{28}$ A. de Angelis, ${ }^{29}$ F. de Palma, ${ }^{15,16}$ C. D. Dermer,${ }^{30}$ S. W. Digel, ${ }^{4}$ L. Di Venere ${ }^{4}$ P. S. Drell, ${ }^{4}$ A. Drlica-Wagner,${ }^{4}$ R. Essig ${ }^{4}$ C. Favuzzi ${ }^{15,16}$ S. J. Fegan, ${ }^{17}$ E. C. Ferrara, ${ }^{14}$ W. B. Focke, ${ }^{4}$ A. Franckowiak, ${ }^{4}$ Y. Fukazawa, ${ }^{31}$ S. Funk ${ }^{4}$ P. Fusco, ${ }^{15,16}$ F. Gargano, ${ }^{16}$ D. Gasparrini,${ }^{22,23}$ S. Germani, ${ }^{12,13}$ N. Giglietto, ${ }^{15,16}$ F. Giordano, ${ }^{15,16}$ M. Giroletti, ${ }^{28}$ T. Glanzman, ${ }^{4}$ G. Godfrey, ${ }^{4}$ G. A. Gomez-Vargas, ${ }^{32,33,34}$ I. A. Grenier, ${ }^{20}$ S. Guiriec, ${ }^{14}$ M. Gustafsson, ${ }^{35}$ D. Hadasch, ${ }^{18}$ M. Hayashida, ${ }^{4,36}$ A. B. Hill,,${ }^{4,37}$ D. Horan, ${ }^{17}$ X. Hou, ${ }^{38}$ R. E. Hughes, ${ }^{3}$ Y. Inoue, ${ }^{4}$ E. Izaguirre, ${ }^{4}$ T. Jogler, ${ }^{4}$ T. Kamae, ${ }^{4}$ J. Knödlseder, ${ }^{39,40}$ M. Kuss, ${ }^{10}$ J. Lande, ${ }^{4}$ S. Larsson, ${ }^{25,26,41}$ L. Latronico, ${ }^{42}$ F. Longo, ${ }^{6,7}$ F. Loparco, ${ }^{15,16}$ M. N. Lovellette, ${ }^{30}$ P. Lubrano, ${ }^{12,13}$ D. Malyshev, ${ }^{4}$ M. Mayer, ${ }^{1}$ M. N. Mazziotta, ${ }^{16}$ J. E. McEnery, ${ }^{14,43}$ P. F. Michelson, ${ }^{4}$ W. Mitthumsiri, ${ }^{4}$ T. Mizuno, ${ }^{44}$ A. A. Moiseev, ${ }^{45,43}$ M. E. Monzani ${ }^{4}$ A. Morselli, ${ }^{32}$ I. V. Moskalenko, ${ }^{4}$ S. Murgia,${ }^{4}$ T. Nakamori, ${ }^{46}$ R. Nemmen, ${ }^{14}$ E. Nuss, ${ }^{24}$ T. Ohsugi, ${ }^{44}$ A. Okumura, ${ }^{4,47}$ N. Omodei, ${ }^{4}$ M. Orienti, ${ }^{28}$ E. Orlando, ${ }^{4}$ J. F. Ormes,${ }^{48}$ D. Paneque, ${ }^{49,4}$ J. S. Perkins,${ }^{14,50,45}$ M. Pesce-Rollins,${ }^{10}$ F. Piron,${ }^{24}$ G. Pivato, ${ }^{9}$ S. Rainò, ${ }^{15,16}$ R. Rando ${ }^{8,9}$ M. Razzano, ${ }^{10,51}$ S. Razzaque, ${ }^{52}$ A. Reimer, ${ }^{11,4}$ O. Reimer, ${ }^{11,4}$ R. W. Romani, ${ }^{4}$

M. Sánchez-Conde, ${ }^{4}$ A. Schulz, ${ }^{1}$ C. Sgrò,${ }^{10}$ J. Siegal-Gaskins,${ }^{53}$ E. J. Siskind,${ }^{54}$ A. Snyder, ${ }^{4}$ G. Spandre, ${ }^{10}$ P. Spinelli, ${ }^{15,16}$ D. J. Suson, ${ }^{55}$ H. Tajima, ${ }^{4,47}$ H. Takahashi, ${ }^{31}$ J. G. Thayer, ${ }^{4}$ J. B. Thayer, ${ }^{4}$ L. Tibaldo, ${ }^{4}$ M. Tinivella, ${ }^{10}$ G. Tosti, ${ }^{12,13}$ E. Troja,${ }^{14}$ Y. Uchiyama, ${ }^{56}$ T. L. Usher, ${ }^{4}$ J. Vandenbroucke, ${ }^{4}$ V. Vasileiou, ${ }^{24}$ G. Vianello, ${ }^{4,57}$ V. Vitale ${ }^{32,58}$ B. L. Winer, ${ }^{3, \S}$ K. S. Wood, ${ }^{30}$ M. Wood, ${ }^{4}$ Z. Yang, ${ }^{25,26}$ G. Zaharijas, ${ }^{6,59}$ and S. Zimmer ${ }^{25,26}$

\section{(Fermi-LAT Collaboration)}

\author{
${ }^{1}$ Deutsches Elektronen Synchrotron DESY, D-15738 Zeuthen, Germany \\ ${ }^{2}$ Space Sciences Laboratory, 7 Gauss Way, University of California, Berkeley, California 94720-7450, USA \\ ${ }^{3}$ Department of Physics, Center for Cosmology and AstroParticle Physics, \\ The Ohio State University, Columbus, Ohio 43210, USA
}

${ }^{4}$ W. W. Hansen Experimental Physics Laboratory, Kavli Institute for Particle Astrophysics and Cosmology,

Department of Physics and SLAC National Accelerator Laboratory, Stanford University, Stanford, California 94305, USA

${ }^{5}$ Università di Pisa and Istituto Nazionale di Fisica Nucleare, Sezione di Pisa, I-56127 Pisa, Italy

${ }^{6}$ Istituto Nazionale di Fisica Nucleare, Sezione di Trieste, I-34127 Trieste, Italy

${ }^{7}$ Dipartimento di Fisica, Università di Trieste, I-34127 Trieste, Italy

${ }^{8}$ Istituto Nazionale di Fisica Nucleare, Sezione di Padova, I-35131 Padova, Italy

${ }^{9}$ Dipartimento di Fisica e Astronomia “G. Galilei," Università di Padova, I-35131 Padova, Italy

${ }^{10}$ Istituto Nazionale di Fisica Nucleare, Sezione di Pisa, I-56127 Pisa, Italy

${ }^{11}$ Institut für Astro- und Teilchenphysik and Institut für Theoretische Physik, Leopold-Franzens-Universität Innsbruck, A-6020 Innsbruck, Austria

${ }^{12}$ Istituto Nazionale di Fisica Nucleare, Sezione di Perugia, I-06123 Perugia, Italy

${ }^{13}$ Dipartimento di Fisica, Università degli Studi di Perugia, I-06123 Perugia, Italy

${ }^{14}$ NASA Goddard Space Flight Center, Greenbelt, Maryland 20771, USA

${ }^{15}$ Dipartimento di Fisica "M. Merlin” dell'Università e del Politecnico di Bari, I-70126 Bari, Italy

${ }^{16}$ Istituto Nazionale di Fisica Nucleare, Sezione di Bari, 70126 Bari, Italy

${ }^{17}$ Laboratoire Leprince-Ringuet, École polytechnique, CNRS/IN2P3, F-91128 Palaiseau, France

${ }^{18}$ Institut de Ciències de l'Espai (IEEE-CSIC), Campus UAB, 08193 Barcelona, Spain

${ }^{19}$ INAF-Istituto di Astrofisica Spaziale e Fisica Cosmica, I-20133 Milano, Italy

${ }^{20}$ Laboratoire AIM, CEA-IRFU/CNRS/Université Paris Diderot, Service d'Astrophysique, CEA Saclay, 91191 Gif sur Yvette, France

${ }^{21}$ Center for Earth Observing and Space Research, College of Science, George Mason University,

Fairfax, Virginia 22030, resident at Naval Research Laboratory, Washington, DC 20375, USA

${ }^{22}$ Agenzia Spaziale Italiana (ASI) Science Data Center, I-00044 Frascati (Roma), Italy

${ }^{23}$ Istituto Nazionale di Astrofisica - Osservatorio Astronomico di Roma, I-00040 Monte Porzio Catone (Roma), Italy

${ }^{24}$ Laboratoire Univers et Particules de Montpellier, Université Montpellier 2, CNRS/IN2P3, F-34095 Montpellier, France 
${ }^{25}$ Department of Physics, Stockholm University, AlbaNova, SE-106 91 Stockholm, Sweden

${ }^{26}$ The Oskar Klein Centre for Cosmoparticle Physics, AlbaNova, SE-106 91 Stockholm, Sweden

${ }^{27}$ The Royal Swedish Academy of Sciences, Box 50005, SE-104 05 Stockholm, Sweden

${ }^{28}$ INAF Istituto di Radioastronomia, 40129 Bologna, Italy

${ }^{29}$ Dipartimento di Fisica, Università di Udine and Istituto Nazionale di Fisica Nucleare, Sezione di Trieste, Gruppo Collegato di Udine, I-33100 Udine, Italy

${ }^{30}$ Naval Research Laboratory, Space Science Division, Washington, DC 20375-5352, USA

${ }^{31}$ Department of Physical Sciences, Hiroshima University, Higashi-Hiroshima, Hiroshima 739-8526, Japan

${ }^{32}$ Istituto Nazionale di Fisica Nucleare, Sezione di Roma “Tor Vergata," I-00133 Roma, Italy

${ }^{33}$ Departamento de Física Teórica, Universidad Autónoma de Madrid, Cantoblanco, E-28049 Madrid, Spain

${ }^{34}$ Instituto de Física Teórica IFT-UAM/CSIC, Universidad Autónoma de Madrid, Cantoblanco, E-28049 Madrid, Spain

${ }^{35}$ Service de Physique Theorique, Universite Libre de Bruxelles (ULB), Bld du Triomphe, CP225, 1050 Brussels, Belgium

${ }^{36}$ Department of Astronomy, Graduate School of Science, Kyoto University, Sakyo-ku, Kyoto 606-8502, Japan

${ }^{37}$ School of Physics and Astronomy, University of Southampton, Highfield, Southampton SO17 1BJ, United Kingdom

${ }^{38}$ Centre d'Études Nucléaires de Bordeaux Gradignan, IN2P3/CNRS,

Université Bordeaux 1, BP120, F-33175 Gradignan Cedex, France

${ }^{39}$ CNRS, IRAP, F-31028 Toulouse cedex 4, France

${ }^{40}$ GAHEC, Université de Toulouse, UPS-OMP, IRAP, Toulouse, France

${ }^{41}$ Department of Astronomy, Stockholm University, SE-106 91 Stockholm, Sweden

${ }^{42}$ Istituto Nazionale di Fisica Nucleare, Sezione di Torino, I-10125 Torino, Italy

${ }^{43}$ Department of Physics and Department of Astronomy, University of Maryland, College Park, Maryland 20742, USA

${ }^{44}$ Hiroshima Astrophysical Science Center, Hiroshima University, Higashi-Hiroshima, Hiroshima 739-8526, Japan

${ }^{45}$ Center for Research and Exploration in Space Science and Technology (CRESST) and NASA Goddard Space Flight Center, Greenbelt, Maryland 20771, USA

${ }^{46}$ 1-4-12 Kojirakawa-machi, Yamagata-shi 990-8560, Japan

${ }^{47}$ Solar-Terrestrial Environment Laboratory, Nagoya University, Nagoya 464-8601, Japan

${ }^{48}$ Department of Physics and Astronomy, University of Denver, Denver, Colorado 80208, USA

${ }^{49}$ Max-Planck-Institut für Physik, D-80805 München, Germany

${ }^{50}$ Department of Physics and Center for Space Sciences and Technology,

University of Maryland Baltimore County, Baltimore, Maryland 21250, USA

${ }^{51}$ Santa Cruz Institute for Particle Physics, Department of Physics and Department of Astronomy and Astrophysics, University of California at Santa Cruz, Santa Cruz, California 95064, USA

${ }^{52}$ Department of Physics, University of Johannesburg, University of Johannesburg, Auckland Park 2006, South Africa

${ }^{53}$ California Institute of Technology, MC 314-6, Pasadena, California 91125, USA

${ }^{54}$ NYCB Real-Time Computing Inc., Lattingtown, New York 11560-1025, USA

${ }^{55}$ Department of Chemistry and Physics, Purdue University Calumet, Hammond, Indiana 46323-2094, USA

${ }^{56}$ 3-34-1 Nishi-Ikebukuro,Toshima-ku, Tokyo 171-8501, Japan

${ }^{57}$ Consorzio Interuniversitario per la Fisica Spaziale (CIFS), I-10133 Torino, Italy

${ }^{58}$ Dipartimento di Fisica, Università di Roma "Tor Vergata," I-00133 Roma, Italy

${ }^{59}$ International Center for Theoretical Physics, Strada Costiera 11, Trieste 34151, Italy

(Received 23 May 2013; published 22 October 2013)

Weakly interacting massive particles (WIMPs) are a theoretical class of particles that are excellent dark matter candidates. WIMP annihilation or decay may produce essentially monochromatic $\gamma$ rays detectable by the Fermi Large Area Telescope (LAT) against the astrophysical $\gamma$-ray emission of the Galaxy. We have searched for spectral lines in the energy range 5-300 GeV using 3.7 years of data, reprocessed with updated instrument calibrations and an improved energy dispersion model compared to the previous Fermi-LAT Collaboration line searches. We searched in five regions selected to optimize sensitivity to different theoretically motivated dark matter density distributions. We did not find any globally significant lines in our a priori search regions and present $95 \%$ confidence limits for annihilation cross sections of self-conjugate WIMPs and decay lifetimes. Our most significant fit occurred at $133 \mathrm{GeV}$ in our smallest search region and had a local significance of 3.3 standard deviations, which translates to a global significance of 1.5 standard deviations. We discuss potential systematic effects in this search, and examine the feature at $133 \mathrm{GeV}$ in detail. We find that the use both of reprocessed data and of additional

\footnotetext{
*albert.143@osu.edu

†elliott@slac.stanford.edu

‡echarles@slac.stanford.edu

§winer@mps.ohio-state.edu
} 
information in the energy dispersion model contributes to the reduction in significance of the linelike feature near $130 \mathrm{GeV}$ relative to significances reported in other works. We also find that the feature is narrower than the LAT energy resolution at the level of 2 to 3 standard deviations, which somewhat disfavors the interpretation of the $133 \mathrm{GeV}$ feature as a real WIMP signal.

\section{INTRODUCTION}

Cosmological studies indicate that $\sim 27 \%$ of the energy density of the Universe is nonbaryonic dark matter (DM) [1]. While substantial astrophysical evidence exists for DM through its gravitational interaction, little has been determined about the composition of the DM or its properties. In a popular class of models [2-4], the DM is a weakly interacting massive particle (WIMP), denoted by $\chi$. In many models WIMP pairs can annihilate into a photon $(\gamma)$ and a second particle $(X)$-for example, $\gamma \gamma, \gamma Z$, or $\gamma H$. (See Refs. [5,6] for reviews on WIMPs and indirect DM detection.) Since DM is strongly constrained to be electrically neutral, it has no direct coupling to photons. Thus the process $\chi \chi \rightarrow \gamma X$ occurs only through higherorder loops, resulting in a branching fraction that is only $\sim 10^{-4}-10^{-1}[7-11]$. If a WIMP annihilates to $\gamma X$, the photons are monochromatic with rest-frame energy

$$
E_{\gamma}=m_{\chi}\left(1-\frac{m_{X}^{2}}{4 m_{\chi}^{2}}\right)
$$

An intrinsic broadening occurs if $X$ is an unstable particle like $Z$. In the case of $X$ being a second photon, the $\gamma$-ray line appears at the mass of the WIMP particle. WIMP decay could also produce a monochromatic signal [12,13] (e.g., $\chi \rightarrow \gamma \nu$ [14]). Additionally, $\gamma$ rays created in WIMP annihilations via internal bremsstrahlung could produce a sharp spectral feature [15], but this channel is not considered in this search. We assume WIMPs in the Milky Way are nonrelativistic $\left(v \sim 10^{-3} c\right)$; therefore these signals should be approximately monochromatic in the lab frame as well. In this paper we present a search for monochromatic $\gamma$ rays from WIMP annihilation or decay.

The Fermi Gamma-ray Space Telescope (Fermi), with its main instrument, the Large Area Telescope (LAT) [16], is exploring the $\gamma$-ray sky in the energy range from $20 \mathrm{MeV}$ to above $300 \mathrm{GeV}$. Previous searches by the LAT Collaboration for $\gamma$-ray lines were published using 11 months and 2 years of LAT data [17,18]. For the search presented here, we use 3.7 years of LAT data that have been reprocessed with updated calibrations. Additionally, two analysis improvements enhance the sensitivity of this search relative to our previous papers: (i) we included an event-by-event estimate of the energy reconstruction quality in our parametrization of the energy dispersion, and (ii) we selected regions of interest (ROIs) a priori to maximize the sensitivity based on different DM density profiles.
Detections of a linelike feature at $130 \mathrm{GeV}$ have been reported in the literature. This feature is reported to be strongly correlated with the Galactic center region [19-22], and also with nearby galaxy clusters [23] and unassociated LAT sources $[24,25]$. The feature has not been seen in the vicinity of nearby dwarf galaxies [26]. However, such a signal is expected to be much fainter than in the Galactic center. Potential instrumental effects and a similar feature detected in the bright $\gamma$-ray emission from cosmic-ray (CR) interactions in Earth's upper atmosphere (the Limb) have also been discussed [27-29]. A systematic investigation of the spatial morphology of the $130 \mathrm{GeV}$ feature and other linelike features in the Galactic plane is presented in Ref. [30]. In addition to the results from our search for $\gamma$-ray lines, we also include a detailed investigation of this feature in the Galactic center region and the Limb.

Section II describes the LAT instrument and the event selections used for this analysis. Section III describes the choice of ROIs, and Sec. IV describes the development of the energy dispersion model. Section V presents the fitting procedure. Section VI summarizes the instrumental and methodological uncertainties associated with this search. Section VII presents the fitting results and derives upper limits for DM annihilation and decay assuming several potential distributions of DM. Section VIII describes studies performed specifically to explore the linelike feature at $133 \mathrm{GeV}$ detected with moderate local significance in our smallest search region. Finally, Sec. IX discusses our results and conclusions.

\section{LAT INSTRUMENT AND EVENT SELECTION}

The LAT is a pair conversion telescope, which converts $\gamma$ rays to $e^{+} e^{-}$pairs that are tracked in the instrument. The data analysis is event based; individual events are reconstructed, and their energies and directions are estimated from the reconstructed data. Rates of CR backgrounds can exceed the $\gamma$-ray rates by factors of up to $10^{4}$, requiring powerful event selection criteria to obtain relatively pure $\gamma$-ray samples.

The LAT consists of three detector subsystems: a tracker/converter to promote pair conversion and measure the directions of the resulting particles, a calorimeter composed of 8.6 radiation lengths of $\mathrm{CsI}(\mathrm{Tl})$ scintillation crystals that provides an energy resolution of $\Delta E / E \sim 10 \%$ at $100 \mathrm{GeV}$, and an anticoincidence detector of plastic scintillator tiles that surrounds the tracker and is key in CR background rejection. The tracker comprises $18 x-y$ layers of silicon strip detectors; the front 12 layers are interleaved 
TABLE I. Event selections in the PASS 7 and PASS 7REP iterations of the LAT event reconstruction and classification algorithms.

\begin{tabular}{lll}
\hline \hline PASS 7 Selection & PASS 7REP Selection & \multicolumn{1}{c}{ Recommended Use/Notes ${ }^{\text {a,b }}$} \\
\hline P7_TRANSIENT & P7REP_TRANSIENT & Analysis of short-duration $(<200$ s $)$ transient sources \\
P7_SOURCE & P7REP_SOURCE & Analysis of point sources and regions of bright diffuse emission \\
P7_CLEAN & P7REP_CLEAN & Analysis of regions of faint diffuse emission \\
P7_ULTRACLEAN & P7REP_ULTRACLEAN & Nearly identical selection to CLEAN for energies above a few GeV \\
\hline \hline
\end{tabular}

${ }^{a}$ The selections are nested; each is a strict subset of the previous one.

${ }^{\mathrm{b}}$ Although the selection criteria are identical between the PASS 7 and PASS 7REP versions, the events selected differ due to changes in the calibration constants used during event reconstruction.

with thin (3\% of a radiation length) tungsten converter foils, then the next four layers are interleaved with thick (18\% of a radiation length) foils, and the final two layers have no converter foils. Detailed descriptions of the LAT and of its performance can be found elsewhere [16,31].

Iterations of the LAT event reconstruction and classification algorithms have been grouped into so-called "passes." The first five "passes" occurred before launch. For the first three years of the mission, data were processed with the PASS 6 version of the algorithms. Since then, the data have been processed with PASS 7, which consists of the same event reconstruction algorithms, but the event classification criteria were updated to account for knowledge gained since launch. (Before switching to PASS 7, the LAT Collaboration also reprocessed all of the original PASS 6 data with the PASS 7 algorithms, so as to provide a single, coherent data set.) Finally, in 2012 and 2013, we reprocessed the data using almost exactly the same PASS 7 algorithms, but with updated calibration constants in the reconstruction algorithms to make the PASS 7REP data sets. More details about the data reprocessing are provided in Appendix A and Ref. [32]. All of these data, as well as more information about recommended usage, are publicly available from the Fermi Science Support Center. ${ }^{1}$

Each pass of the algorithms implements several different event selection criteria that are optimized for different types of analyses. In PASS 7, the LAT Collaboration implemented four nested event selections that provide varying levels of CR background rejection. The names of the event selections, as well as the types of analyses they are optimized for, are listed in Table I. The nomenclature convention for the various event selections is to provide the pass version and the name of the event selection criteria (e.g., P7REP_CLEAN). Associated with each event selection are instrument response functions (IRFs) that parametrize the LAT performance. As our understanding of the instrument improves, from time to time the LAT Collaboration updates the IRFs for the various event selections. The IRF names indicate which data set they are associated with, as well as a version number (e.g., P7REP_CLEAN_V10). More

\footnotetext{
${ }^{1}$ The LAT photon data are available at http://fermi.gsfc.nasa .gov/ssc/data/access/.
}

details about the event reconstruction, event selection criteria, and IRFs can be found in Ref. [31]. ${ }^{2}$

As discussed in Sec. II A, we use only the P7REP_CLEAN event selection for the line search. For certain studies of potential systematic biases, we compare the P7REP CLEAN sample against either the P7REP_TRANSIENT or P7REP_SOURCE sample. Finally, as part of our examination of the feature near $130 \mathrm{GeV}$, we compare the P7REP_CLEAN sample with the P7_CLEAN sample used in previous papers [19-22].

\section{A. Event selection}

We searched for the presence of $\gamma$-ray lines between 5 and $300 \mathrm{GeV}$; to include spectral sideband regions in the energy ranges for all the fits (see Sec. VA), we extracted data in the range $2.6-541 \mathrm{GeV}$.

We used the P7REP_CLEAN event selection for data acquired between August 4, 2008, and April 18, 2012. We used this more selective event class for this analysis because the CR background contamination in the P7REP_SOURCE class can dominate over the diffuse $\gamma$-ray contribution at high Galactic latitudes. We sought to minimize CR background contamination because Monte Carlo (MC) studies have shown that reconstructing CRs (and especially protons and other hadrons) under the assumption that they are $\gamma$ rays can produce a variety of spectral features (see Appendix D, Sec. D 5). Further discussion about the CR background contamination in P7SOURCE and P7CLEAN can be found in Ref. [31]; the results change little for the reprocessed P7REP_SOURCE and P7REP_CLEAN event selections. The $\gamma$-ray effective collecting area (or simply "effective area") on axis for the P7REP_CLEAN event selection ranges from 6500 to $7200 \mathrm{~cm}^{2}$ over the energy range of interest.

We selected both a Celestial data set (for the line search) and a data set corresponding to the Limb (as a control region); see Table II. The Limb is a very bright $\gamma$-ray source of secondary $\gamma$ rays produced by CR interactions in the upper atmosphere. Figure 1 shows a schematic of the

\footnotetext{
${ }^{2}$ Performance details for all the iterations of the event reconstruction and classification algorithms used since launch are available at http://www.slac.stanford.edu/exp/glast/groups/ canda/lat_Performance.htm.
} 
TABLE II. Summary table of data selections.

\begin{tabular}{lcc}
\hline \hline Selection & Celestial data & Limb data \\
\hline Observation period $^{\mathrm{a}}(\mathrm{s})$ & August 4, 2008-April 4, 2012 & August 4, 2008-October 6, 2012 \\
Mission elapsed time $^{\mathrm{a}}$ & {$[239557447,356434906]$} & {$[239557447,371176784]$} \\
Energy range $(\mathrm{GeV})^{\text {Zenith range }(\mathrm{deg})}$ & {$[2.6,541]$} & {$[2.6,541]$} \\
${\text { Rocking angle range }(\mathrm{deg})^{\mathrm{b}}}_{\text {Data quality cut }^{\mathrm{c}}}$ & $\theta_{\mathrm{z}}<100$ & $111<\theta_{\mathrm{z}}<113$ \\
Source masking (see text) & $\left|\theta_{\mathrm{r}}\right|<52$ & $\left|\theta_{\mathrm{r}}\right|>52$ \\
\hline
\end{tabular}

${ }^{\mathrm{a}}$ Fermi mission elapsed time is defined as seconds since January 1, 2001, 00:00:00 UTC.

${ }^{\mathrm{b}}$ Applied by selecting on ROCK_ANGLE with the gtmktime ScienceTool.

${ }^{\mathrm{c}}$ Standard data quality selection: DATA_QUAL $==1 \& \&$ LAT_CONFIG $==1$ with the gtmktime ScienceTool.

geometry for $\gamma$-ray production in the Limb, as well as the definitions of the zenith angle $\left(\theta_{\mathrm{z}}\right)$, spacecraft rocking angle $\left(\theta_{\mathrm{r}}\right)$, and $\gamma$-ray incidence angle $(\theta)$.

For the Celestial data set, we removed the Limb $\gamma$ rays by selecting only events with $\theta_{z}<100^{\circ}$. We also only used data collected when a small fraction of the LAT field of view (FOV) subtended the Limb by removing times when $\left|\theta_{\mathrm{r}}\right|>52^{\circ}$.

For the Limb data set, we selected a narrow range of zenith angles $\left(111^{\circ}<\theta_{z}<113^{\circ}\right)$. One should note that while Fermi is in normal survey mode [31], the Limb is fairly far off axis $\left(\theta>60^{\circ}\right)$, near the edge of the LAT FOV. This means that the events in the Celestial data set have a quite different $\theta$ distribution than events from the Limb collected during survey mode observations. Since the effective area and energy resolution of the LAT depend strongly on $\theta$, it is important to use a Limb control data set collected when Fermi was not in normal survey mode, but rather was slewed toward the Limb. Therefore, for the Limb data set we reversed the rocking angle criterion and selected times when $\left|\theta_{\mathrm{r}}\right|>52^{\circ}$. This represents $\sim 0.3 \%$ of the livetime of the 3.7 year Celestial data set. Because of the extreme brightness of the limb, the contamination from Celestial $\gamma$ rays is small; it is $<6 \%$ at $3 \mathrm{GeV}$, decreases with energy, and is $<0.5 \%$ for all energies $>6 \mathrm{GeV}$.

The initial steps of the data reduction and all of the exposure calculations were performed with the LAT ScienceTools ${ }^{3}$ version 09-29-00 using the P7REP CLEAN_V10 IRFs. The P7REP_CLEAN_V10 IRFs will not be the set of IRFs recommended for use with P7REP_CLEAN data. The recommended IRFs for use with the reprocessed data will be publicly released in the fall of 2013. The differences between P7REP_CLEAN_V10 and subsequent versions of the P7REP_CLEAN IRFs are very small above $5 \mathrm{GeV}$, and we have verified that their use does not significantly change the results presented in this paper.

In order to limit the contribution to the Celestial data set from discrete $\gamma$-ray sources, we applied an

\footnotetext{
${ }^{3}$ The ScienceTools and documentation are available at http:// fermi.gsfc.nasa.gov/ssc/data/analysis/scitools/overview.html.
}

energy-dependent mask around the 527 point sources in the second Fermi-LAT source catalog (2FGL catalog) [33] detected with greater than $10 \sigma$ significance above $1 \mathrm{GeV}$. The energy scaling of the $68 \%$ containment angle $\left(\theta_{68}\right)$ of the LAT point spread function (PSF) can be modeled as $\theta_{68}(E)=\sqrt{c_{0}^{2}(E / 1 \mathrm{GeV})^{-2 \beta}+c_{1}^{2}}$ [31]. We performed an effective-area-weighted average of the flight-derived P7CLEAN_V6 PSF over incidence angle to obtain the parameters $c_{0}=0.881, c_{1}=0.2016$, and $\beta=0.817$, which give $\theta_{68}=0.31^{\circ}$ and $\theta_{68}=0.20^{\circ}$ at $5 \mathrm{GeV}$ and $300 \mathrm{GeV}$, respectively. We used a source mask radius of $2 \times \theta_{68}(E)$. In each of the ROIs (see Sec. III), this masking removed $\sim 1.5 \%$ of the solid angle and $\sim 10 \%$ of the events. We estimate that the residual contamination from point sources in our energy range constitutes $\leqslant 10 \%$ of the events in our Celestial data set.

Our event selection criteria for the Celestial and Limb data sets are summarized in Table II. Note that we included events through September 2012 in our Limb data set to take

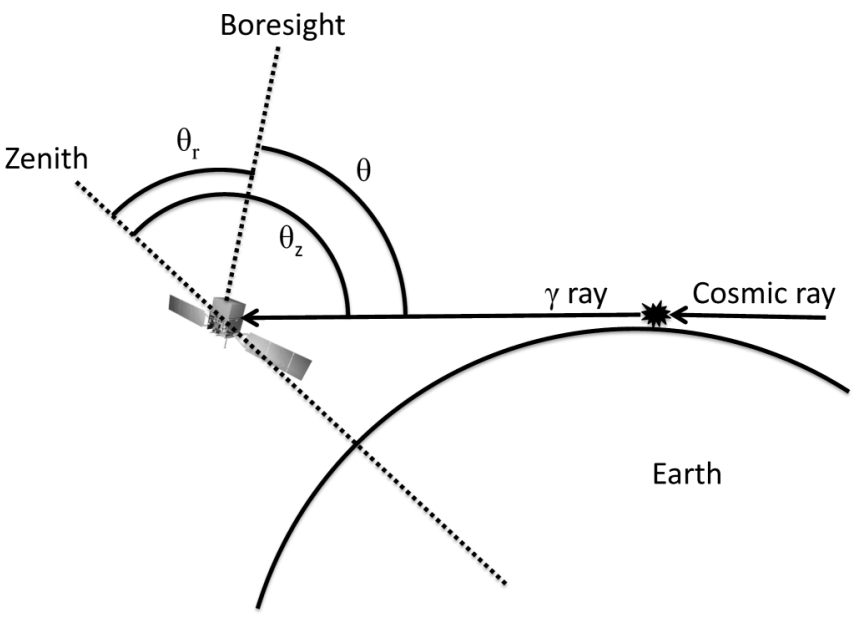

FIG. 1. Schematic of Limb $\gamma$-ray production by CR interactions in the Earth's atmosphere, showing the definitions of the zenith angle $\left(\theta_{\mathrm{z}}\right)$, the spacecraft rocking angle $\left(\theta_{\mathrm{r}}\right)$, and the incidence angle $(\theta)$. The dashed line starts at the center of the Earth. Not drawn to scale. 
TABLE III. Summary of optimized ROIs and J-factor values for each of the four DM density profiles considered for both annihilating and decaying WIMPs.

\begin{tabular}{|c|c|c|c|c|}
\hline Profile & ROI & $\begin{array}{l}\text { Annihilation } \\
\mathrm{J} \text { factor }\left(10^{22} \mathrm{GeV}^{2} \mathrm{~cm}^{-5}\right)\end{array}$ & ROI & $\begin{array}{c}\text { Decay } \\
\mathrm{J} \text { factor }\left(10^{23} \mathrm{GeV} \mathrm{cm}^{-2}\right)\end{array}$ \\
\hline NFW contracted & R3 & 13.9 & $\mathrm{R} 180$ & 2.42 \\
\hline Einasto & R16 & 8.48 & R180 & 2.49 \\
\hline NFW & R41 & 8.53 & $\mathrm{R} 180$ & 2.46 \\
\hline Isothermal & R90 & 6.94 & R180 & 2.80 \\
\hline
\end{tabular}

advantage of events collected during a week-long targeted pointing that included appreciable amounts of time with $\left|\theta_{\mathrm{r}}\right|>52^{\circ}$.

\section{B. Simulated data sets}

To model the response of the LAT, we used several simulated data sets created with a GEANT4-based [34] MC simulation of $\gamma$-ray interactions with the LAT and analyzed using the same event reconstruction algorithms as are applied to the data. We relied on a few particular simulated data sets: (i) the "all-gamma" data set [31], an isotropic distribution of $\gamma$ rays with an $E^{-1}$ spectrum used to generate the standard IRFs; (ii) "isotropic monochromatic" data sets, i.e., isotropic distributions of $\gamma$ rays at specific energies used to generate our model for the energy dispersion; and (iii) an "all-sky background" data set, where the simulation used the Fermi pointing history and the source model included all 2FGL catalog sources, diffuse emission from the Galaxy, and isotropic emission. ${ }^{4}$

\section{REGIONS OF INTEREST}

We have developed a set of five ROIs optimized for sensitivity to WIMP annihilation or decay and four reference models for the distribution of DM in the Galaxy. The details of the optimization procedure are described in Appendix B.

For the distribution of DM in the Galaxy, we consider four smooth parametrizations. The Navarro-Frenk-White (NFW) profile [35],

$$
\rho(r)=\frac{\rho_{s}}{\left(r / r_{s}\right)\left(1+r / r_{s}\right)^{2}},
$$

with $r_{s}=20 \mathrm{kpc}$ has been found to characterize the smooth distribution of DM in simulated halos. The Einasto profile,

$$
\rho(r)=\rho_{s} \exp \left\{-(2 / \alpha)\left[\left(r / r_{s}\right)^{\alpha}-1\right]\right\},
$$

with $r_{s}=20 \mathrm{kpc}$ and $\alpha=0.17$ is favored by more recent cold dark matter (CDM) simulations [36]. We additionally consider an isothermal profile with a central core,

\footnotetext{
${ }^{4}$ Specifically, gal_2yearp7v6_v0.fits and iso_p7v6clean.txt, available at http://fermi.gsfc.nasa.gov/ssc/data/access/lat/ BackgroundModels.html.
}

$$
\rho(r)=\frac{\rho_{s}}{1+\left(r / r_{s}\right)^{2}},
$$

with $r_{s}=5 \mathrm{kpc}$ [37]. Finally, adiabatic contraction of the DM halo due to infall of baryonic matter in the Galactic center region could result in DM density profiles with a much steeper central slope than either the NFW or Einasto profile [38]. We take as a representative of this class of models a contracted NFW profile defined by

$$
\rho(r)=\frac{\rho_{s}}{\left(r / r_{s}\right)^{\gamma}\left(1+r / r_{s}\right)^{3-\gamma}},
$$

with $\gamma=1.3$. For all profiles we determine the normalization of the profile density $\left(\rho_{s}\right)$ by fixing the DM density at the solar radius $\rho\left(r_{\odot}=8.5 \mathrm{kpc}\right)=0.4 \mathrm{GeV} \mathrm{cm}^{-3}$ $[39,40]$.

We defined a set of five ROIs, circular regions of radius $R_{\mathrm{GC}}$ centered on the Galactic center with $|b|<5^{\circ}$ and $|l|>6^{\circ}$ masked, which were optimized for each of the DM density profiles considered. For annihilating DM models we use $R_{\mathrm{GC}}=3^{\circ}$ (R3, optimized for the contracted NFW profile), $16^{\circ}$ (R16, optimized for the Einasto profile), $41^{\circ}$ (R41, NFW), and $90^{\circ}$ (R90, optimized for the isothermal profile), while for decaying DM models we use $R_{\mathrm{GC}}=$ $180^{\circ}$ (R180). We did not apply a source mask for the R3 data set, so we limited the search in R3 to energies greater than $30 \mathrm{GeV}$ (see Appendix B). Above this energy, the composite $\gamma$-ray flux from point sources is much less than the integral flux of the Galactic diffuse emission rate in R3.

Table III summarizes the optimized ROI that was used for each DM halo profile and its associated astrophysical $\mathbf{J}$ factor (i.e., the integral along the line of sight of $\rho(r)^{2}$ for DM annihilation or $\rho(r)$ for DM decay; see Appendix B). We note that the point source masking reduced the annihilation $\mathrm{J}$ factor by $<10 \%$ in each ROI, except for R3, where no point source masking was applied. The counts map of the 3.7-year Celestial data set in the R180 ROI with outlines of the other four ROIs is shown in Fig. 2.

\section{MODELING OF THE ENERGY DISPERSION}

The algorithms for reconstructing LAT events provide three estimates of the event energy: one based on a parametric correction of the raw energy measured by the calorimeter, a second based on a maximum likelihood fit using the correlations between the raw energy in the calorimeter 


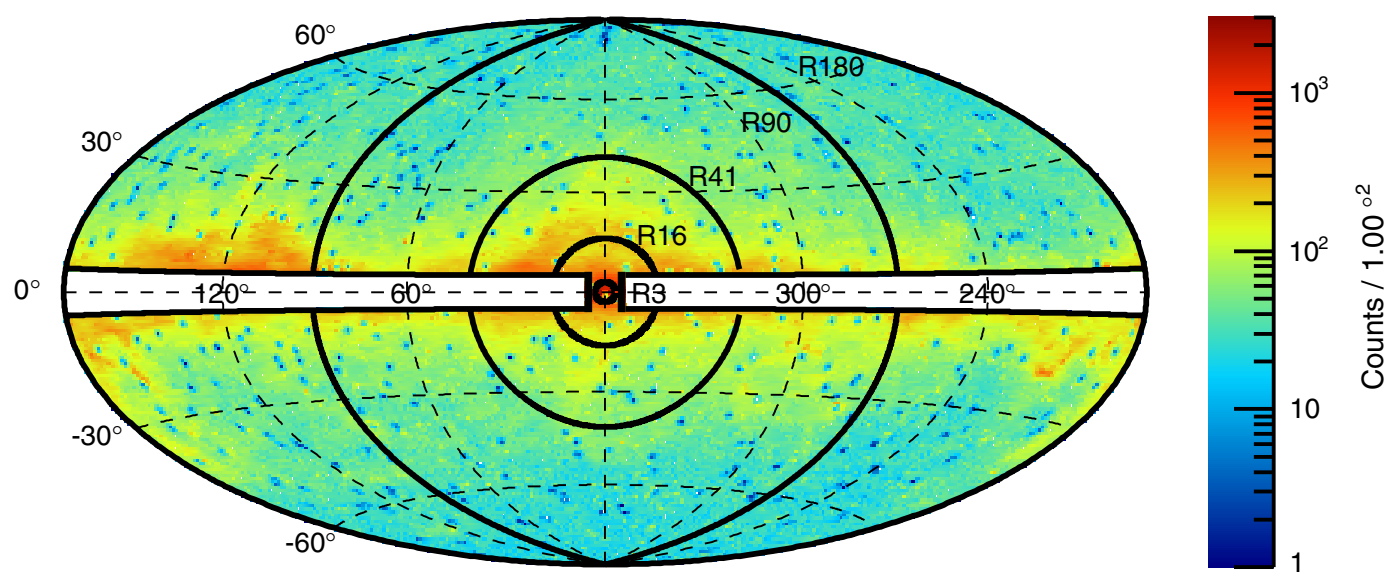

FIG. 2 (color online). Counts map for the line-search data set binned in $1^{\circ} \times 1^{\circ}$ spatial bins in the R180 ROI, and plotted in Galactic coordinates using the Hammer-Aitoff projection. The energy range is $2.6-541 \mathrm{GeV}$, and the most significant $2 \mathrm{FGL}$ sources have been removed using an energy-dependent mask (see text). Also shown are the outlines of the other ROIs (R3, R16, R41, and R90) used in this search.

and other event properties, and a third based on a fit to the shower profile in the calorimeter [16]. The likelihoodbased method was found to create narrow features in the LAT energy response that could mimic linelike spectral features, which is the main reason why previous spectral line searches performed by the LAT Collaboration with the PASS 6 data sets used the shower profile energy estimate exclusively $[17,18]$. In the PASS 7 version of the event-level analysis, the result of the likelihood method is ignored, and we use a classification tree analysis to select which of the other two methods is more likely to provide the best energy estimate on an event-by-event basis. The corresponding estimate is the energy assigned. We note that above a few $\mathrm{GeV}$, the shower profile method is typically more accurate than the parametric correction method (the former being selected by the classification tree analysis for $\sim 80 \%$ of the events above $10 \mathrm{GeV}$ ).

The energy assignment algorithm also performs a classification tree analysis to estimate the probability that the energy estimate is within the nominal $68 \%$ containment band for events of that energy and incidence angle $\left(P_{\mathrm{E}}\right)^{5}$

To model the signal from a $\gamma$-ray line, we used a parametrization of the effective energy dispersion of the instrument, i.e., the probability density $D_{\text {eff }}\left(E^{\prime} ; E, \vec{s}\right)$, to measure an energy $E^{\prime}$ for a $\gamma$ ray of (true) energy $E$ and other event parameters, $\vec{s}$. The fraction of the electromagnetic shower contained in the calorimeter can vary significantly event to event. In general, the energy dispersion depends on $\theta$ and the $\gamma$-ray conversion point in the instrument, among other quantities. Furthermore, the $\theta$

\footnotetext{
${ }^{5}$ Available as CTBBESTENERGYPROB in the extended event files available at the Fermi Science Support Center at http:// fermi.gsfc.nasa.gov/ssc/data/access/, and described at http:// fermi.gsfc.nasa.gov/ssc/data/analysis/documentation/Cicerone/ Cicerone_Data/LAT_Data_Columns.html\#ExtendedFile.
}

distribution of the observing time varies across the sky, causing corresponding changes in the effective energy dispersion. These considerations are discussed in more detail in Appendix C, in particular in its Sec. D 5.

When fitting essentially monochromatic lines (i.e., the intrinsic spectrum is much narrower than the instrumental resolution) for a given line energy, $E_{\gamma}$, we expect the distribution of observed energies for a line signal, $C_{\text {sig }}\left(E^{\prime}\right)$, to follow the effective energy dispersion, $D_{\text {eff }}$, so that

$$
\begin{aligned}
C_{\text {sig }}\left(E^{\prime} \mid E_{\gamma}, \vec{s}\right) & =n_{\text {sig }} \int D_{\text {eff }}\left(E^{\prime} ; E, \vec{s}\right) \delta\left(E_{\gamma}-E\right) d E \\
& =n_{\text {sig }} D_{\text {eff }}\left(E^{\prime} ; E_{\gamma}, \vec{s}\right),
\end{aligned}
$$

where $n_{\text {sig }}$ is the number of observed signal events, which we treat as a free parameter in the fitting (see Sec. V). ${ }^{6}$

Following the approach used in previous line searches published by the LAT Collaboration, we use a sum of Gaussians to parametrize the energy dispersion at any given energy, averaging over the LAT FOV and combining events that convert in the front or back sections of the tracker [18]. One notable improvement relative to our previous studies is that the parametrization $D_{\text {eff }}\left(E^{\prime} ; E, P_{\mathrm{E}}\right)$ used in this work includes the energy reconstruction quality estimator, $P_{\mathrm{E}}$. Specifically, we modeled the energy dispersion in $10 P_{\mathrm{E}}$ bins of 0.2 from 0.1 to 0.5 , bins of 0.1 from 0.5 to 0.7 , and bins of 0.05 from 0.7 to 1 . The P7REP_CLEAN event class only includes events with $P_{\mathrm{E}}>0.1$.

\footnotetext{
${ }^{6}$ This assumption breaks down when the intrinsic width of the $\gamma$-ray emission becomes a sizable fraction of the LAT energy resolution. In practical terms, this applies for final states with unstable particles such as $Z \gamma$, in particular for $\gamma$-ray energies at the low end of our search range. We discuss the implications of this in Appendix D, Sec. D 3.
} 


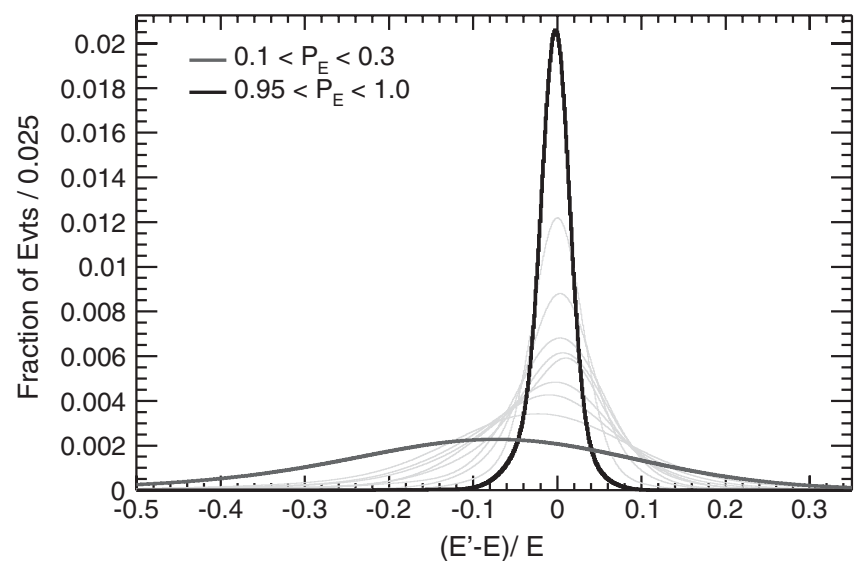

FIG. 3. Energy dispersion model for $100 \mathrm{GeV}$ in all $10 P_{\mathrm{E}}$ bins. The darker lines show the distributions for the smallest and largest values of $P_{\mathrm{E}}$, while the thinner gray lines show the models for the intermediate $P_{\mathrm{E}}$ bins.

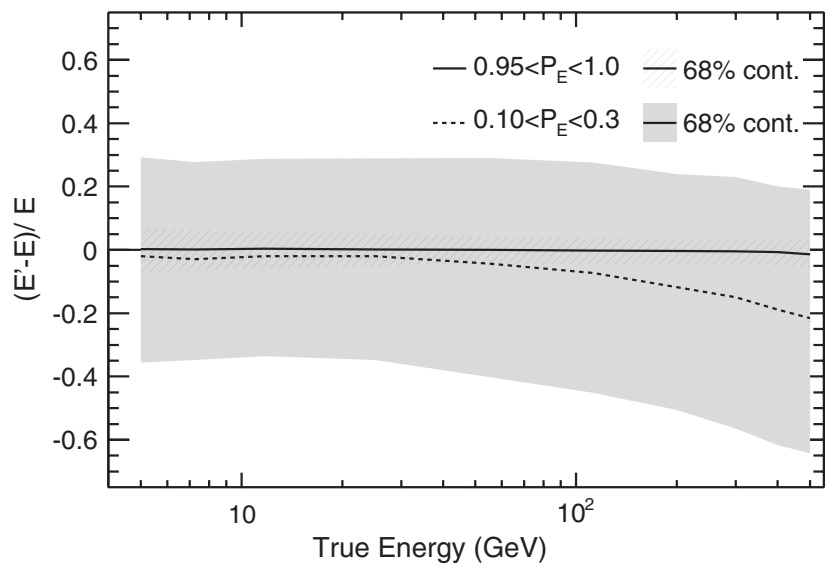

FIG. 4. The bias of the energy dispersion for the lowest (highest) $P_{\mathrm{E}}$ bins is indicated by the dashed (solid) line. The shaded regions show the $68 \%$ containment for the highest and lowest $P_{\mathrm{E}}$ bins.

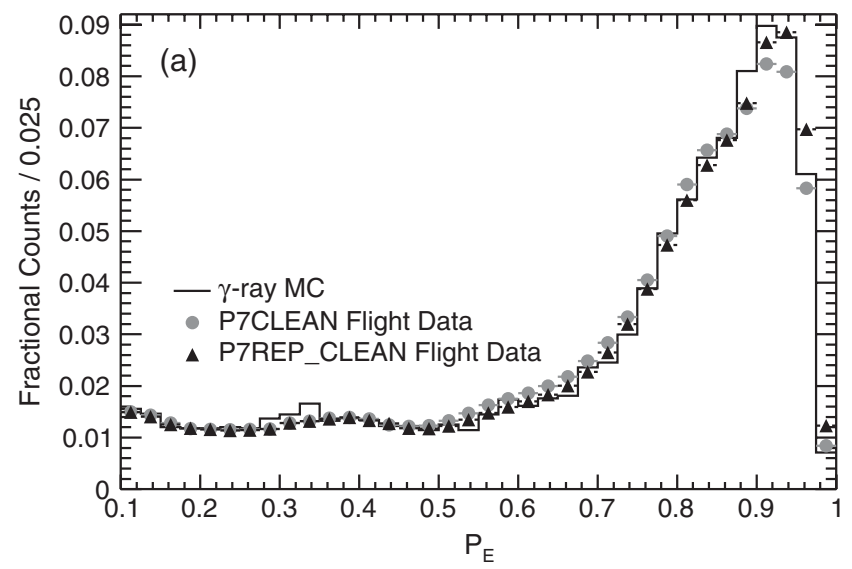

The energy dispersion in each $P_{\mathrm{E}}$ bin was modeled with a triple Gaussian function

$$
D_{\text {eff }}\left(E^{\prime} ; E, P_{\mathrm{E}}\right)=\sum_{k=1}^{3} \frac{a_{k}}{\sigma_{k} \sqrt{2 \pi}} e^{-\left(\left(E^{\prime} / E\right)-\left(1+\mu_{k}\right)\right)^{2} / 2 \sigma_{k}^{2}},
$$

where $a_{3}=1-a_{2}-a_{1}$. To avoid degeneracy between the Gaussians, we constrain the ranges of the $\sigma_{i}$ to ensure that $\sigma_{1}>\sigma_{2}>\sigma_{3}$.

We explicitly determined energy dispersion model parameters for $E$ values of 5, 7, 10, 20, 50, 100, 200 and $300 \mathrm{GeV}$ using "isotropic monochromatic" $\gamma$-ray MC simulations (see Sec. II B) at each of those energies. The systematic uncertainties associated with using these simulations to derive our model are discussed in Appendix D, Sec. D 4. When fitting for a spectral line at $E_{\gamma}$, we interpolated the appropriate energy dispersion parameters. The resulting energy dispersion models at $E=100 \mathrm{GeV}$ in all $10 P_{\mathrm{E}}$ bins are shown in Fig. 3. The bias and 68\% containment of our energy dispersion model as a function of $E$ are shown in Fig. 4. The bias is the fractional deviation of the energy dispersion peak from the true energy.

The distribution of $P_{\mathrm{E}}$ depends on energy and ROI. Figure 5 shows the distribution of $P_{\mathrm{E}}$ in the P7CLEAN and P7REP_CLEAN data sets and the "all-sky background" $\gamma$-ray MC for $|b|>10^{\circ}$. While the agreement between $\mathrm{MC}$ and data is good, overall, there is clearly some discrepancy in the upper half, in $\log (E)$, of our energy range that has been reduced by the reprocessing.

With the addition of $P_{\mathrm{E}}$ as a parameter for the energy dispersion, we are not only testing that a possible line signal effectively follows the energy dispersion, but also that well-reconstructed events are clustered closer to the peak energy. This contributes significant additional information to the likelihood fitting; to quantify the improvement, we compared the effect on sensitivity from modeling the energy dispersion as simply a function of energy ["1D" model, $D\left(E^{\prime} ; E_{\gamma}\right)$, as was done in Ref. [18]] to

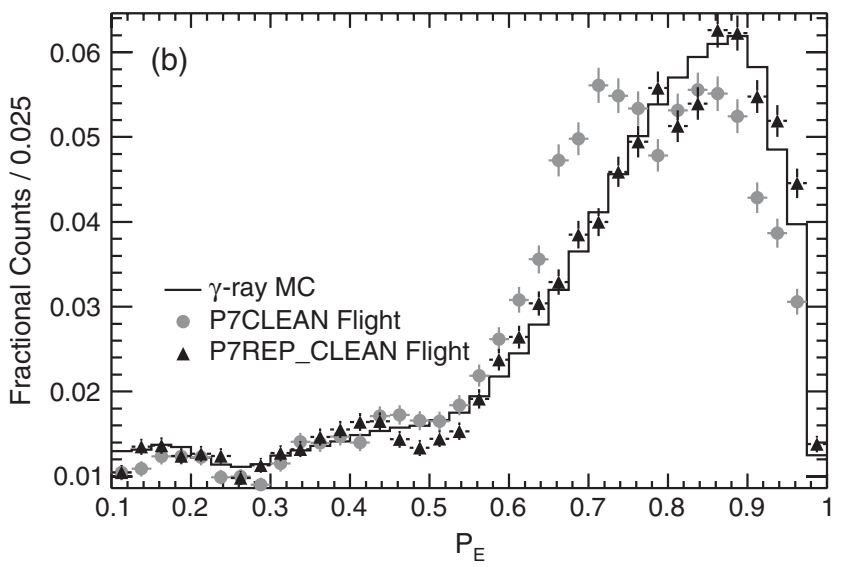

FIG. 5. $\quad P_{\mathrm{E}}$ distributions for flight and all-sky MC data sets (see text) over (a) the entire energy range $(2.6-541 \mathrm{GeV})$, and (b) the energy range $52-541 \mathrm{GeV}$. 
using a model with $P_{\mathrm{E}}$ [“2D” model, $\left.D\left(E^{\prime} ; E_{\gamma}, P_{\mathrm{E}}\right)\right]$ using simulations both with and without a spectral line. Including the extra information, $P_{\mathrm{E}}$, the statistical power is increased by $15 \%$ on average.

\section{FITTING}

\section{A. Fitting method}

We searched for spectral lines by performing maximum likelihood fits in sliding energy intervals in the five ROIs described in Sec. III. Specifically, we fit the count spectra in the energy domain, integrating over each ROI. Given model uncertainties and the relatively uniform coverage of the sky by the LAT, we made several assumptions to simplify the fitting procedure, which are outlined in Appendix C. ${ }^{7}$ We used the RooFit toolkit [41] (version 3.12) to implement the models and perform the likelihood minimization.

In general, we performed unbinned maximum likelihood fits; however, because of the large number of events at the lower end of our energy range, we performed binned fits for energies $<25 \mathrm{GeV}$ to reduce the required computation time. When performing binned fits, we used 60 bins across the fit energy range. Since the bin width is small compared to the instrument resolution, we lose very little information. We confirmed that binned fits yield results nearly identical to unbinned fits by simulating 1000 pseudoexperiments both with and without a signal and fitting for a line at $20 \mathrm{GeV}$.

We fit in narrow, approximately half-decade energy ranges, and therefore approximated the background spectrum, $C_{\mathrm{bkg}}\left(E^{\prime}\right)$, as a single power law with an index $\Gamma_{\mathrm{bkg}}$ that was allowed to float in the fit. We also incorporated the energy dependence of the exposure averaged across each ROI into the background component ${ }^{8}$ by means of an energy-dependent exposure correction $\eta\left(E^{\prime}\right)$ :

$$
C_{\mathrm{bkg}}\left(E^{\prime} \mid \Gamma_{\mathrm{bkg}}, n_{\mathrm{bkg}}\right)=n_{\mathrm{bkg}}\left(\frac{E^{\prime}}{E_{0}}\right)^{-\Gamma_{\mathrm{bkg}}} \eta\left(E^{\prime}\right),
$$

where $E_{0}$ is a reference energy (we used $E_{0}=1 \mathrm{MeV}$ ); $n_{\mathrm{bkg}}$ is the total number of background events, which is a free parameter in the fit; and $\eta\left(E^{\prime}\right)$ is given by Eq. (C18), which includes a normalization constraint that is defined by Eq. (C15). Since both the background and exposure vary smoothly and slowly across our fit ranges, we did not explicitly convolve the above equations with the energy dispersion to derive the expected models for the observed energy; i.e., we assumed $E^{\prime}=E$ in Eq. (8).

\footnotetext{
${ }^{7}$ Since we are using the P7REP_CLEAN event class and combining front- and back-converting events, we suppress the event selection $(\vec{s})$ relative to the notation used in Appendix C.

${ }^{8}$ Any variation across the energy interval in the exposure would introduce an artificial shaping to the background spectrum.
}

Our complete counts model to fit for a line at $E_{\gamma}$ is

$$
\begin{aligned}
C\left(E^{\prime}, P_{\mathrm{E}} \mid \vec{\alpha}\right)= & n_{\mathrm{sig}} D_{\mathrm{eff}}\left(E^{\prime} ; E_{\gamma}, P_{\mathrm{E}}\right) w^{\mathrm{ROI}}\left(P_{\mathrm{E}}\right) \\
& +n_{\mathrm{bkg}}\left(\frac{E^{\prime}}{E_{0}}\right)^{-\Gamma_{\mathrm{bkg}}} \eta\left(E^{\prime}\right) w^{\mathrm{ROI}}\left(P_{\mathrm{E}}\right),
\end{aligned}
$$

where the model parameters $\vec{\alpha}$ are $E_{\gamma}, \Gamma_{\mathrm{bkg}}, n_{\mathrm{sig}}$, and $n_{\mathrm{bkg}}$. Note that we fit for $n_{\text {sig }}$ independent of any DM model assumption; we then assumed a specific DM profile and calculated the $\mathrm{J}$ factor in the ROI in order to solve for the annihilation cross section or decay lifetime given the magnitude of the exposure in that ROI (see Sec. VII).

Since we incorporated $P_{\mathrm{E}}$ in the signal model, we included the distributions of $P_{\mathrm{E}}, w\left(P_{\mathrm{E}}\right)$. For each fit in a specific ROI and energy interval, we took the $P_{\mathrm{E}}$ distributions for both signal and background from all of the data in the ROI and energy range; i.e., $w_{\mathrm{bkg}}\left(P_{\mathrm{E}}\right)=w_{\text {sig }}\left(P_{\mathrm{E}}\right)=$ $w^{\mathrm{ROI}}\left(P_{\mathrm{E}}\right)$. The small effect from this approximation is discussed in Appendix D, Sec. D 4.

The energy interval for a fit at $E_{\gamma}$ in this search was $\pm 6 \sigma_{E}\left(E_{\gamma}\right)$, where $\sigma_{E}$ is the on-axis LAT energy resolution at the fit energy. (Specifically, $\sigma_{E}$ is the half-width of the $\pm 34 \%$ containment about the peak value of the energy dispersion for on-axis events.) The interval was broadened from the previous LAT analysis [18] to reduce the statistical uncertainty of $\Gamma_{\mathrm{bkg}}$. This consequently reduced the uncertainty of $n_{\text {sig }}$, because the maximum likelihood values of the parameters are correlated in the fits. As discussed in Ref. [20], the significance of the fit has a slight dependence on interval size. However, for energy ranges wider than $\sim 12 \sigma_{E}$, the change in significance is small compared to the expected statistical variation. Additionally, fitting in wider intervals may reduce the validity of approximating the background as a power law. However, we do not find that this approximation induces a large systematic effect (see Appendix D, Sec. D 7 b).

Each fit was performed at a specific energy $E_{\gamma}$ as opposed to letting the line energy float in the fit. The spacing between adjacent fit energies is half the energy resolution. Simulations show that with this choice, the loss of signal for potential lines offset with respect to our search grid is small; at worst we underfit $n_{\text {sig }}$ by less than $10 \%$. We constrained $n_{\text {sig }}$ to be positive to avoid unphysical measurements as well as negative likelihoods.

We calculate the local significance by taking the square root of the test statistic $\left(s_{\text {local }}=\sqrt{T S}\right)$, which is defined as twice the difference in the log likelihood between the maximum likelihood hypothesis and the null hypothesis:

$$
T S=2 \ln \frac{\mathcal{L}\left(n_{\text {sig }}=n_{\text {sig, best }}\right)}{\mathcal{L}\left(n_{\text {sig }}=0\right)} .
$$

We expect at least ten (and usually many more) effective background events (see Sec. VIA) for each energy range and ROI considered in the fits, so the Gaussian 
approximation for the application of Chernoff's [42] theorem to predict a $\chi^{2}$ distribution (for one bounded degree of freedom) of $T S$ is well justified.

\section{B. Global significance}

We fit lines for 88 different $E_{\gamma}$ values each in R16, R41, R90, and R180, and 44 values in R3 (where we only fit for $E_{\gamma}>30 \mathrm{GeV}$ ), for a total of 396 fits. Given this number of trials, it is reasonable to expect some of the fits to indicate apparently significant values for the number of signal events even if the underlying data are purely background. If our trials involved independent data samples, we could translate a local $\mathrm{p}$ value to a global (i.e., post-trial) $\mathrm{p}$ value using $p_{\text {global }}=1-\left(1-p_{\text {local }}\right)^{396}$.

However, our samples were not independent. In fact, converting between the $T S$ and global significance, $s_{\text {global }}$ (i.e., $p_{\text {global }}$ expressed as a significance relative to the standard deviation of a Gaussian distribution, $\sigma$ ), is complicated because

(1) The energy ranges overlap, meaning the fits were not all independent.

(2) The ROIs are nested in those of larger radii (see Sec. III).

To estimate $s_{\text {global }}$, we simulated 1000 background-only realizations of our search. For each realization, we generated five independent samples corresponding to the nonoverlapping parts of our five ROIs (i.e., we generated samples representing R3, R16 without R3, R41 without R16, and so on). For each sample, we simulated background-only events with $\Gamma_{\mathrm{bkg}}=2.4$ and the exposure corrections from R3. For simplicity, and to reduce the computational time required, we omitted $P_{\mathrm{E}}$ from the model used for these realizations. We merged the independent samples to obtain simulated data sets matching our ROIs, with the correct amount of overlapping events.

For each realization, we performed all 396 fits for a line signal at the various energies in all of the ROIs and

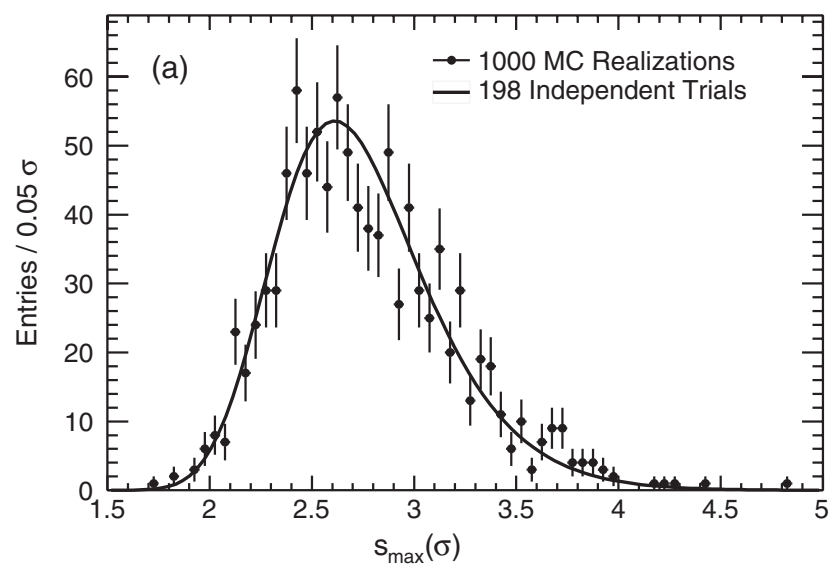

extracted the largest $s_{\text {local }}$ value obtained by any of the fits $\left(s_{\max }\right)$. Empirically, we found that the distribution of $s_{\max }$ values for each realization was well modeled by the expected distribution for $n_{\mathrm{t}}$ trials where the $T S$ distribution follows a $\chi^{2}$ distribution with one bounded degree of freedom [see Fig. 6(a)]:

$f\left(s_{\max }\right)=\frac{n_{\mathrm{t}}}{2}\left(\delta\left(s_{\max }\right)+\frac{e^{-s_{\max }^{2} / 2 .}}{\sqrt{2 \pi}}\right)\left(1-\frac{1}{2} P_{\chi^{2}}\left(s_{\max }^{2}, 1\right)\right)^{\left(n_{\mathrm{t}}-1\right)}$.

The best-fit number of independent trials was $n_{\mathrm{t}}=$ $198 \pm 6$. Therefore, we estimate that our search consists of 198 effective independent trials and calculate the relation to convert from $s_{\text {local }}$ to $s_{\text {global }}$ accordingly [see Fig. 6(b)].

We also extracted the largest $T S$ value obtained by any of the fits in each ROI and fit for the number of independent trials for that ROI. In each case, we found that the best-fit $n_{\mathrm{t}, \mathrm{ROI}}$ was about $60 \%$ of the number of trials actually performed, or slightly more that one trial for each step of $\sigma_{E}$.

Summing the best-fit number of independent trials from the five ROIs gives 243, while empirically we found $n_{\mathrm{t}}=198$. This suggests that the data sets for the ROIs are largely independent, i.e., the overlap between the ROIs only reduces the effective number of trials by a factor of 0.81 . This is reasonable, given that we gain a factor of $\sim 6$ events going from R3 to R16, 3.2 going from R16 to R41, $\sim 2.2$ going from $\mathrm{R} 41$ to $\mathrm{R} 90$, and $\sim 1.6$ going from $\mathrm{R} 90$ to $\mathrm{R} 180$.

Finally, we note that this conversion between $s_{\text {local }}$ and $s_{\text {global }}$ is only applicable to the specific search using $0.5 \sigma_{E}$ energy steps and five nested ROIs. Accordingly, we do not quote global signficances for fits made on control samples or with other event selections in the course of studying potential systematic biases.

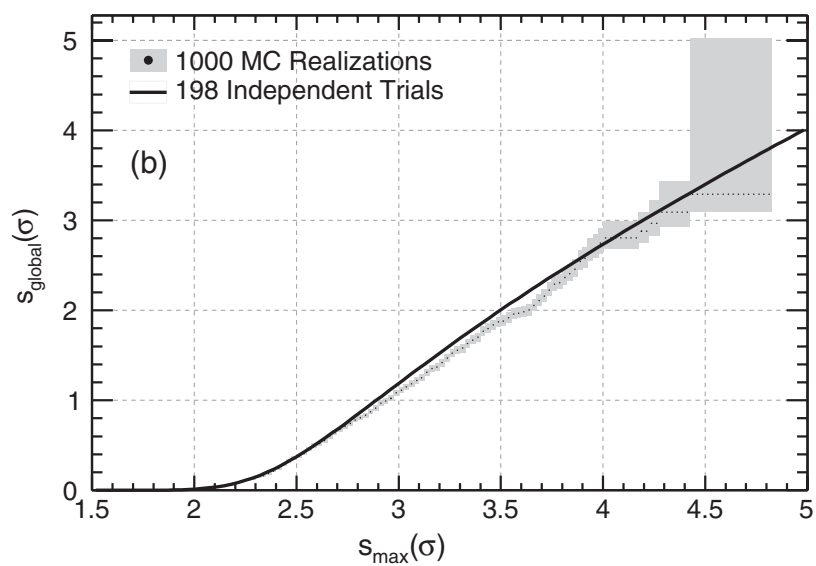

FIG. 6. Derivation of global significance versus local significance given our scan over energy in $0.5 \sigma_{E}$ steps and five ROIs: (a) Distribution of the largest $s_{\text {local }}$ values obtained in any ROI at any energy from 1000 MC realizations (points) and the best-fit independent trials curve. (b) The corresponding $s_{\text {local }}$ to $s_{\text {global }}$ transformation. 


\section{SYSTEMATIC UNCERTAINTIES}

In this section, we introduce and summarize systematic uncertainties related to our search for $\gamma$-ray lines. Detailed discussions of each issue can be found in Appendix D. A discussion of systematic studies performed near $E_{\gamma} \approx 130 \mathrm{GeV}$ is postponed until Sec. VIII.

We consider three classes of systematic uncertainties:

(1) Uncertainties that enter in the conversion between the fit number of signal counts, $n_{\text {sig }}$, and the inferred fluxes. These uncertainties induce a corresponding uncertainty in the estimated model fluxes and upper limits on those fluxes, but do not affect fit significances. We quantify these in terms of the relative uncertainty of the exposure, $\delta \mathcal{E} / \mathcal{E}$. These are discussed in Appendix D, Sec. D 1.

(2) Uncertainties that would scale the fit estimates of the number of signal counts (i.e., affect fit significances and upper limits) but would not otherwise induce or mask a signal. These primarily consist of errors in signal model parametrization. We quantify these in terms of the relative uncertainty of the number of signal counts, $\delta n_{\text {sig }} / n_{\text {sig. }}$. These are discussed in Appendix D, Secs. D 2-D 4.

(3) Uncertainties that could mask a true signal or induce a false signal. We quantify these in terms of the induced fractional signal, $f$ (discussed in Sec. VIA). These uncertainties are discussed in Appendix D, Secs. D 5-D 7.

\section{A. Induced fractional signal}

Many types of systematic uncertainties that could affect this analysis induce narrow spectral features of a fixed fractional size, which we call "induced fractional signals." For example, unmodeled energy-dependent variations in the effective area at the $10 \%$ level would induce features at the same level in the counts spectrum. Therefore, it is useful to consider the signal-to-background ratio of any feature in addition to the statistical significance.

When quantifying the signal-to-background ratio of narrow features, we are more concerned with the background under the signal peak than with the total background in the fit energy range. Therefore, it is useful to consider the "effective background" $b_{\text {eff }}$, which can be calculated in terms of the signal and background probability density functions $F_{\text {sig }}$ and $F_{\text {bkg }}$ (which are just $C_{\text {sig }}$ and $C_{\mathrm{bkg}}$ normalized to unit values, see Appendix C) and the total number of events in the fit range, $\mathrm{N}$ :

$$
b_{\mathrm{eff}}=N \int \frac{F_{\mathrm{sig}}\left(E^{\prime}\right) F_{\mathrm{bkg}}\left(E^{\prime}\right)}{F_{\mathrm{sig}}\left(E^{\prime}\right)+F_{\mathrm{bkg}}\left(E^{\prime}\right)} d E^{\prime} .
$$

The integral is performed over the fit energy interval.

The $T S$ is closely related to $b_{\text {eff }}$, and we find that the following relation holds to within 5\% for fits to both flight data and MC simulations:

$$
b_{\mathrm{eff}} \simeq \frac{n_{\mathrm{sig}}^{2}}{T S} .
$$

As stated above, it is useful to report the magnitude of potential systematic uncertainty in terms of "fractional signal" $f$, i.e., the ratio of signal counts to effective background counts:

$$
f=\frac{n_{\text {sig }}}{b_{\text {eff }}} \simeq \frac{T S}{n_{\text {sig }}}
$$

TABLE IV. Summary of systematic effects. As stated in the text, we quote either the relative uncertainty of the exposure $(\delta \mathcal{E} / \mathcal{E})$, the relative uncertainty of the number of signal events

\begin{tabular}{|c|c|c|c|}
\hline \multirow{2}{*}{$\begin{array}{l}\text { Systematic } \\
\text { Effective area scale }\end{array}$} & \multicolumn{2}{|c|}{ Effect } & \multirow{2}{*}{$\frac{\text { Section }}{\text { D } 1}$} \\
\hline & & $\delta \mathcal{E} / \mathcal{E}= \pm 0.1$ & \\
\hline \multirow[t]{2}{*}{ Averaging exposure over ROI } & (R3) & $|\delta \mathcal{E} / \mathcal{E}|<0.01$ & D 1 \\
\hline & $\left(\mathrm{R} 180, E_{\gamma}=300 \mathrm{GeV}\right)$ & $\delta \mathcal{E} / \mathcal{E}= \pm 0.13$ & D 1 \\
\hline$E_{\gamma}$ grid spacing & & $\delta n_{\mathrm{sig}} / n_{\mathrm{sig}}={ }_{-0.1}^{+0.0}$ & VA \\
\hline Energy resolution & & $\delta n_{\text {sig }} / n_{\text {sig }}= \pm 0.07$ & D 2 \\
\hline Broadening from $\mathrm{Z}$ width & $\left(E_{\gamma}=68 \mathrm{GeV}\right)$ & $\delta n_{\mathrm{sig}} / n_{\mathrm{sig}}=-0.07$ & D 3 \\
\hline$P_{\mathrm{E}}$ distribution variation & & $\delta n_{\text {sig }} / n_{\text {sig }}= \pm 0.01$ & D 4 \\
\hline Energy dispersion model $\theta$ variation & & $\delta n_{\text {sig }} / n_{\text {sig }}= \pm 0.02$ & D 4 \\
\hline \multirow[t]{2}{*}{ CR contamination } & (R3) & $|\delta f|<0.005$ & D 5 \\
\hline & (R180) & $\delta f= \pm 0.014$ & D 5 \\
\hline Point source contamination & & $|\delta f|<0.005$ & D 6 \\
\hline \multirow[t]{2}{*}{ Effective area variations } & $\left(E_{\gamma}=5 \mathrm{GeV}\right)$ & $\delta f= \pm 0.005$ & D 7 a \\
\hline & $\left(E_{\gamma}>100 \mathrm{GeV}\right)$ & $\delta f= \pm 0.025$ & D 7 a \\
\hline \multirow{3}{*}{ Astrophysical background modeling } & $\left(\mathrm{R} 180, E_{\gamma}=30 \mathrm{GeV}\right)$ & $\delta f= \pm 0.005$ & D $7 \mathrm{~b}$ \\
\hline & $\left(\mathrm{R} 180, E_{\gamma}>100 \mathrm{GeV}\right)$ & $\delta f= \pm 0.011$ & D $7 \mathrm{~b}$ \\
\hline & (R3) & $\delta f= \pm 0.019$ & D $7 \mathrm{c}$ \\
\hline
\end{tabular}
$\left(\delta n_{\text {sig }} / n_{\text {sig }}\right)$, or the uncertainty of the induced fractional signal $(\delta f)$. We give representative values when the magnitude of the effect depends on energy, or varies between ROIs. 
TABLE V. Total magnitude of systematic effects, by ROI and energy. We obtained these estimates by adding in quadrature the magnitudes of all the potential uncertainties on $\delta \mathcal{E} / \mathcal{E}$, $\delta n_{\text {sig }} / n_{\text {sig }}$, and $\delta f$ for each ROI.

\begin{tabular}{lcccccc}
\hline \hline Quantity & Energy & \multicolumn{1}{c}{$\mathrm{R} 3$} & $\mathrm{R} 16$ & $\mathrm{R} 41$ & $\mathrm{R} 90$ & $\mathrm{R} 180$ \\
\hline$\delta \mathcal{E} / \mathcal{E}$ & $5 \mathrm{GeV}$ & \pm 0.10 & \pm 0.10 & \pm 0.11 & \pm 0.12 & \pm 0.14 \\
$\delta \mathcal{E} / \mathcal{E}$ & $300 \mathrm{GeV}$ & \pm 0.10 & \pm 0.10 & \pm 0.12 & \pm 0.13 & \pm 0.16 \\
$\delta n_{\text {sig }} / n_{\text {sig }}$ & $\mathrm{All}$ & +0.07 & +0.07 & +0.07 & +0.07 & +0.07 \\
$\delta f$ & $5 \mathrm{GeV}$ & \pm 0.020 & \pm 0.020 & \pm 0.008 & \pm 0.008 & \pm 0.008 \\
$\delta f$ & $50 \mathrm{GeV}$ & \pm 0.024 & \pm 0.024 & \pm 0.015 & \pm 0.015 & \pm 0.015 \\
$\delta f$ & $300 \mathrm{GeV}$ & \pm 0.032 & \pm 0.032 & \pm 0.035 & \pm 0.035 & \pm 0.035 \\
\hline \hline
\end{tabular}

The most practical aspect of this formulation is that it allows us to quickly and easily convert between systematically induced fractional signal and $T S$ for a given search region and energy.

Furthermore, from the above equations, we can see that for a given fractional signal, the local significance in-

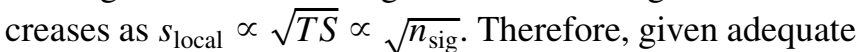
statistics, a small fractional signal can become highly statistically significant.

\section{B. Summary of systematic uncertainties}

Table IV summarizes the systematic effects discussed in Appendix D. In Table $\mathrm{V}$ we have grouped the effects on $\delta \mathcal{E} / \mathcal{E}, \delta n_{\text {sig }} / n_{\text {sig }}$ and $\delta f$ for each ROI.

The systematic uncertainties related to the exposure, when summed in quadrature, can reach up to $\delta \mathcal{E} / \mathcal{E}=$ 0.16 for the R180 and R90 ROIs. However, as stated earlier, they do not affect the signal significance. Furthermore, they only have a minor impact on the limits on $\Phi_{\gamma \gamma}$ and $\langle\sigma v\rangle$, as they are less than $40 \%$ of the expected statistical variations in limits, which are typically $40 \%-50 \%$.

The uncertainties of the energy dispersion modeling could cause us to underestimate a true signal, or inflate a statistical fluctuation. These range over $-0.12<\delta n_{\text {sig }} /$ $n_{\text {sig }}<0.07$. In other words, we might estimate a true $5 \sigma$ signal to be only $4.4 \sigma$, or inflate a $3 \sigma$ fluctuation to be $3.2 \sigma$. These uncertainties also only have a minor impact on the analysis, as even for $5 \sigma$ signals they result in systematic errors that are less than the expected statistical fluctuations, which are about $1 \sigma$.

Uncertainties that can induce or mask a signal can be more problematic. In Table $\mathrm{V}$, these range in magnitude from $\delta f=0.008$ at low energies up to $\delta f=0.035$ at high energies. However, because of increased statistics in the larger ROIs at low energies, even a $f=0.01$ induced signal can become highly statistically significant. We will discuss this question further in Sec. VII. In summary, at energies up to $100 \mathrm{GeV}$, the dominant source of potential systematic bias for the smaller ROIs (R3, R16) is the modeling of the astrophysical backgrounds as a power law, while for the larger ROIs it is CR contamination. Above $100 \mathrm{GeV}$, because of the limited statistics of the
Limb control sample, uncertainties of potential features in the effective area dominate the systematic uncertainties.

\section{FITTING RESULTS AND UPPER LIMITS}

We have performed a scan for spectral lines from 5-300 GeV in the five ROIs described in Sec. III and find no globally significant lines. Figure 7 shows the local fit significance for each of the fit energies and all five ROIs; all of the fits are below $2 \sigma$ global significance. As shown in Fig. 8 , the distribution of the $s_{\text {local }}$ values from our line search is well modeled by the null hypothesis expectation according to Chernoff's theorem [42].

The two most statistically significant fits were in R180 at $6.3 \mathrm{GeV}$, with $s_{\text {local }}=3.1 \sigma$ and $f=0.010 \pm 0.002$, and in $\mathrm{R} 3$ at $135 \mathrm{GeV}$, with $s_{\text {local }}=3.2 \sigma$ (corresponding to $\left.s_{\text {global }}=1.5 \sigma\right)$ and $f=0.58 \pm 0.18$, where $f$ is the effective signal fraction at the line energy [Eq. (14)]. Although

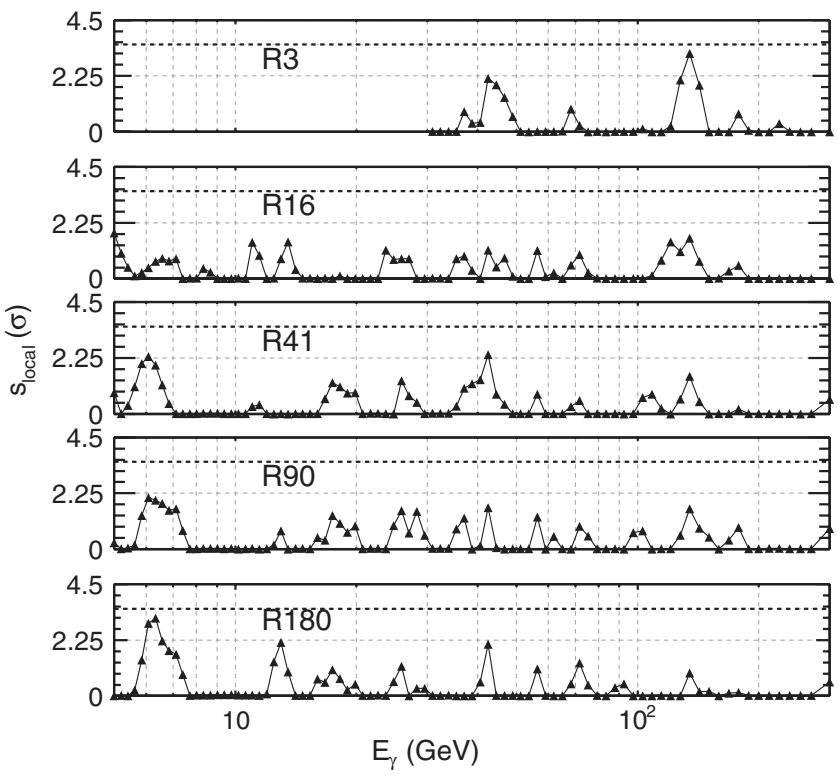

FIG. 7. Local fit significance vs line energy in all five ROIs. Note that $n_{\text {sig }}$ was required to be non-negative. The dashed line at the top of the plot indicates the local significance corresponding to the $2 \sigma$ global significance derived with the method described in Sec. V B. 


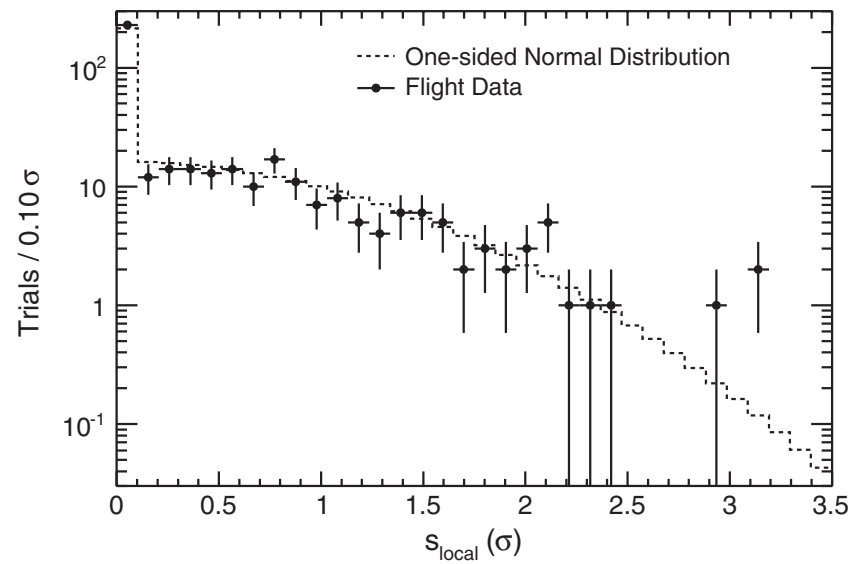

FIG. 8. Local fit significance distribution from our line search. The dashed line shows the expected distribution from the null hypothesis. Note that $n_{\text {sig }}$ was bounded to be positive.

the fit at $6.3 \mathrm{GeV}$ in $\mathrm{R} 180$ has a relatively large $T S$ value, the signal fraction is similar to the expected systematic uncertainty of $\delta f= \pm 0.008$ (see Table V) for R180 at that energy. A fine scan $\left(0.1 \sigma_{E}\right.$ steps) near $135 \mathrm{GeV}$ in $\mathrm{R} 3$ found the largest significance at $133 \mathrm{GeV}$, with $s_{\text {local }}=$ $3.3 \sigma$. We discuss the results near $133 \mathrm{GeV}$ in considerably more detail in Sec. VIII.

Since no globally significant lines were detected, we have derived 95\% confidence level (C.L.) upper limits on the $\gamma$-ray flux from spectral lines $\left(\Phi_{\gamma \gamma}\right)$. We set upper limits on $n_{\text {sig }}\left(E_{\gamma}\right)$ at the point where the log-likelihood changes by $1.36(2.71 / 2)$ with respect to the maximum. Then, using the magnitude of the averaged exposure in each ROI at the fit line energy $\left[\mathcal{E}_{\mathrm{ROI}}\left(E_{\gamma}\right)\right]$, we can convert the $95 \%$ C.L. upper limit on $n_{\text {sig }}\left(E_{\gamma}\right)$ to the $95 \%$ C.L. upper limit on $\Phi_{\gamma \gamma}\left(E_{\gamma}\right)$, using

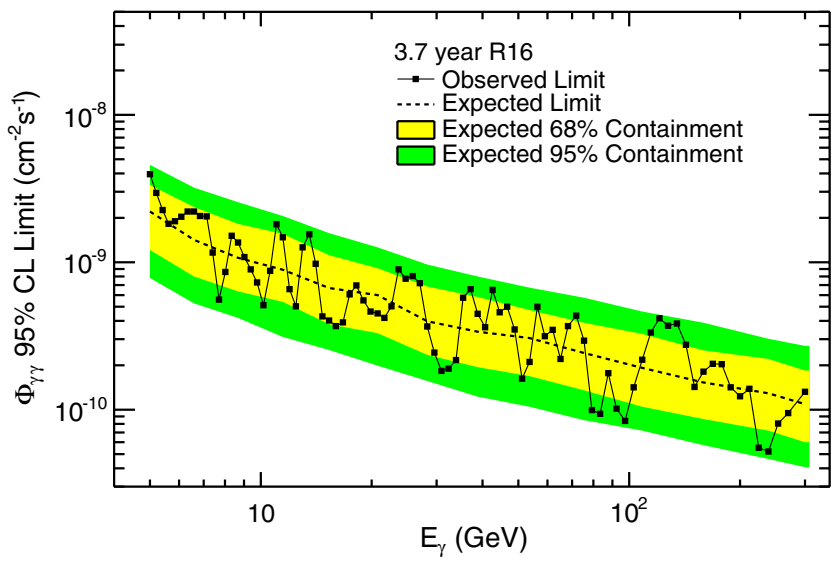

FIG. 9 (color online). $95 \%$ C.L. $\Phi_{\gamma \gamma}$ in the R16 ROI (black). Yellow (green) bands show the 68\% (95\%) expected containment derived from 1000 single-power-law (no DM) MC simulations. The dashed lines show the median expected limits from those simulations.

$$
\Phi_{\gamma \gamma}\left(E_{\gamma}\right)=\frac{n_{\mathrm{sig}}\left(E_{\gamma}\right)}{\mathcal{E}_{\mathrm{ROI}}\left(E_{\gamma}\right)} \text {. }
$$

Note that we solve for the $\Phi_{\gamma \gamma}\left(E_{\gamma}\right)$ limits generally. If $\Phi_{\gamma \gamma}\left(E_{\gamma}\right)$ is associated with DM annihilation or decay, the corresponding annihilation cross section or decay lifetime can be solved for using specific DM model parameters (e.g., J factors).

Figure 9 shows the flux upper limits in the R16 (Einastooptimized) ROI. Also shown are the expected limits and expected $68 \%$ and $95 \%$ containment bands derived from 1000 single-power-law (no DM) MC simulations with $\Gamma_{\mathrm{bkg}}=2.4$. Therefore, these containment bands represent the expected statistical variation of a power-law distribution normalized to the number of events in the data set.

Using Eq. (B5) with $\frac{d N_{\gamma}}{d E}\left(E_{\gamma}\right)=2 \delta\left(E_{\gamma}-E^{\prime}\right)$ and $E_{\gamma}=m_{\chi}$, we solve for the corresponding upper limits on $\langle\sigma v\rangle_{\gamma \gamma}$, which are shown in Fig. 10 for the R3, R16, R41, and R90 ROIs for the contracted NFW, Einasto, NFW, and isothermal profiles, respectively. When directly comparable, the upper limits on $\langle\sigma v\rangle_{\gamma \gamma}$ derived by Weniger [20] are also shown. Note that the contracted NFW limits are not compared, since significantly different ROIs were used in this search compared to the search in Ref. [20]. Also, the isothermal limits are not compared, since different values of $r_{s}$ were used.

R180 is optimized for searches for spectral lines from WIMP decays (e.g., $\chi \rightarrow \gamma \nu$ ). The flux upper limits are related to the lifetime $\left(\tau_{\gamma \nu}\right)$ lower limits via Eq. (B6) with $\frac{d N_{\gamma}}{d E}\left(E_{\gamma}\right)=\delta\left(E_{\gamma}-E^{\prime}\right)$ and $m_{\chi}=2 E_{\gamma}$, which are shown in Fig. 11.

We present the flux upper limits in all five ROIs and the relevant DM annihilation or decay limits explicitly in Appendix E. Recall that we limited our search to energies greater than $30 \mathrm{GeV}$ in $\mathrm{R} 3$ (see Sec. III).

The limits presented do not include systematic errors. As stated in Sec. VIB, the uncertainties of the exposure $(|\delta \mathcal{E} / \mathcal{E}|<0.16)$ and the energy dispersion modeling $\left(\delta n_{\text {sig }} / n_{\text {sig }}={ }_{-0.12}^{+0.06}\right)$ contribute negligibly to the limits when considered in quadrature with the statistical uncertainties. On the other hand, the inferred uncertainties of $\delta f$ from Table $\mathrm{V}$ can become significantly larger than the statistical uncertainties at lower energies and for the larger ROIs. In fact, the uncertainty of $\delta f$ from Table Vequals the expected statistical uncertainty at $10 \mathrm{GeV}$ (for R16 and $\mathrm{R} 41$ ), $30 \mathrm{GeV}$ (for R90), and $70 \mathrm{GeV}$ (for R180). Empirically, the limits presented in Figs. 10 and 11 generally lie within the expected statistical variations, indicating that the systematic uncertainties are not dominating the statistical uncertainties.

\section{THE LINELIKE FEATURE NEAR 133 GEV}

The most significant fit from our search for spectral lines is for $E_{\gamma}=135 \mathrm{GeV}$ in our smallest ROI, R3 (see 

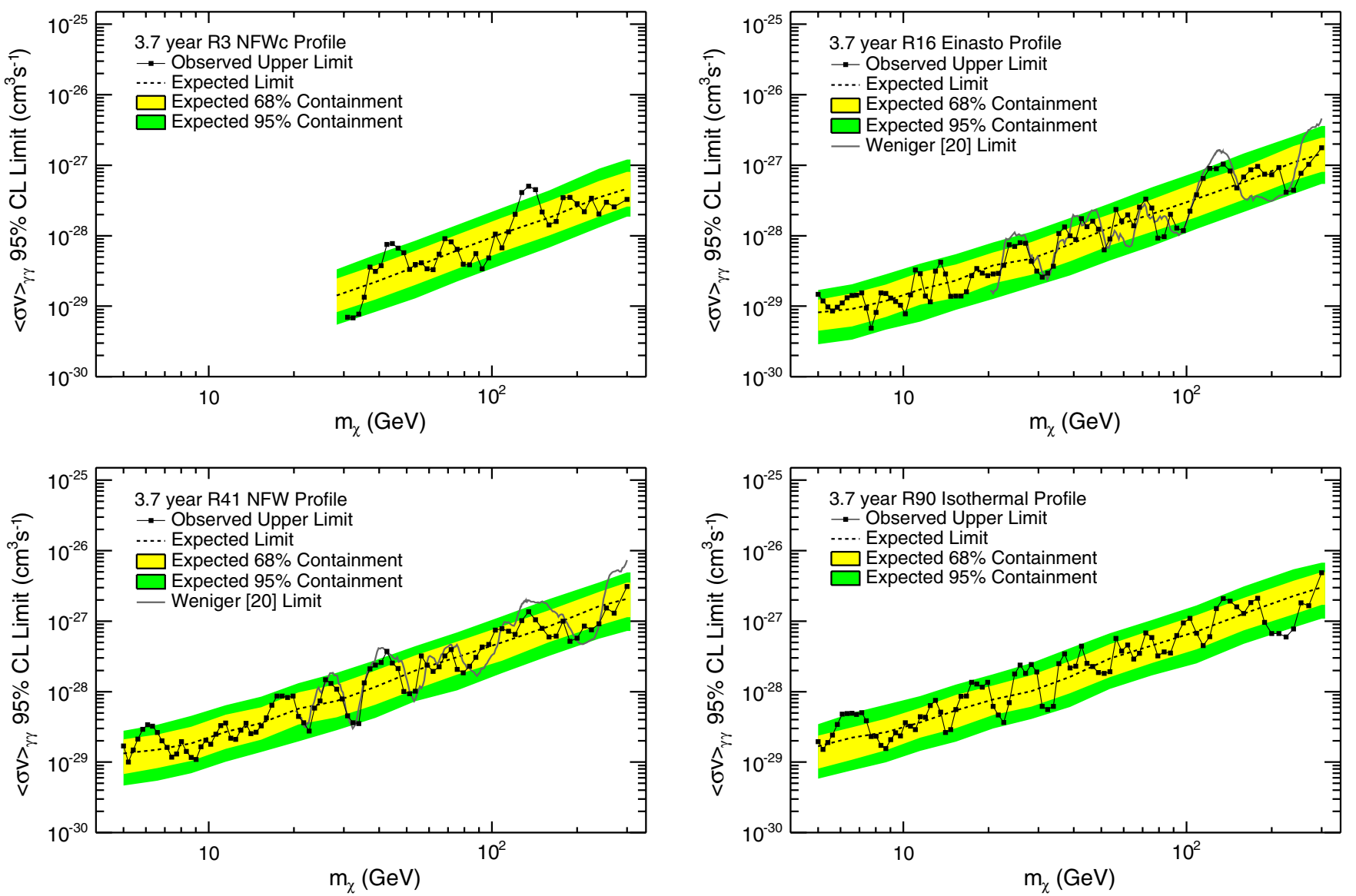

FIG. 10 (color online). $95 \%$ C.L. $\langle\sigma v\rangle_{\gamma \gamma}$ upper limits for each DM profile considered in the corresponding optimized ROI. Yellow (green) bands show the 68\% (95\%) expected containment derived from 1000 single-power-law (no DM) MC simulations. The dashed lines show the median expected limits from those simulations. The solid gray line shows the limits derived by Weniger [20] (an independent search for spectral lines from 20-300 GeV) when comparable ROIs and identical DM density profiles were used.

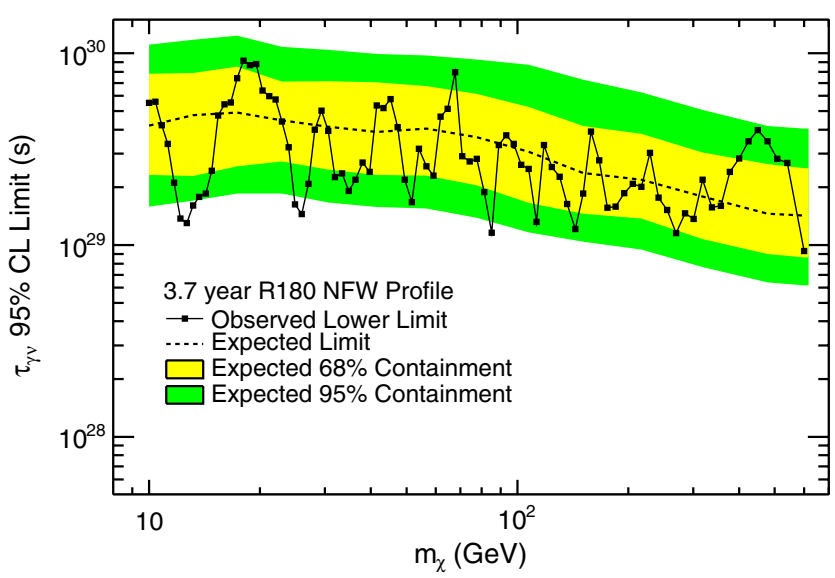

FIG. 11 (color online). $95 \%$ C.L. $\tau_{\gamma \nu}$ lower limits in R180 for a NFW profile. Yellow (green) bands show the 68\% (95\%) expected containment derived from 1000 single-power-law (no DM) MC simulations. The dashed lines show the median expected limits from those simulations.
Sec. VII). A fine scan $\left(0.1 \sigma_{E}\right.$ steps $)$ around this energy in R3 found the largest significance at $133 \mathrm{GeV}$, with $s_{\text {local }}=3.3 \sigma$. The finer scan reduces the potential negative bias from the grid spacing to $\delta n_{\text {sig }} / n_{\text {sig }}<0.02$ but contributes to the trials factor. Using the procedure described in Sec. VB, we estimate that if we had used $0.1 \sigma_{E}$ steps everywhere, the effective number of independent trials would be $n_{\mathrm{t}}=295 \pm 9$, with which we extract $s_{\text {global }}=1.5 \sigma$.

This is the same feature that has been reported in the unreprocessed PASS 7 data at $130 \mathrm{GeV}$ [19-22]. The feature has shifted from $130 \mathrm{GeV}$ to $133 \mathrm{GeV}$ in the reprocessed data, as expected from the application of improved calibrations (see Appendix A). In the rest of this section, we discuss the $133 \mathrm{GeV}$ feature in detail.

\section{A. Evolution of the $133 \mathrm{GeV}$ feature with different data sets and signal models}

We studied how using reprocessed data and the 2D energy dispersion model (see Sec. IV) affects the significance of the observed feature in the two smallest ROIs 
(i.e., where the significances were the greatest): R3, optimized for a contracted NFW profile, and R16, optimized for the Einasto profile. Recall that for the R16 data set, we removed events near bright 2FGL sources (see Sec. II A).
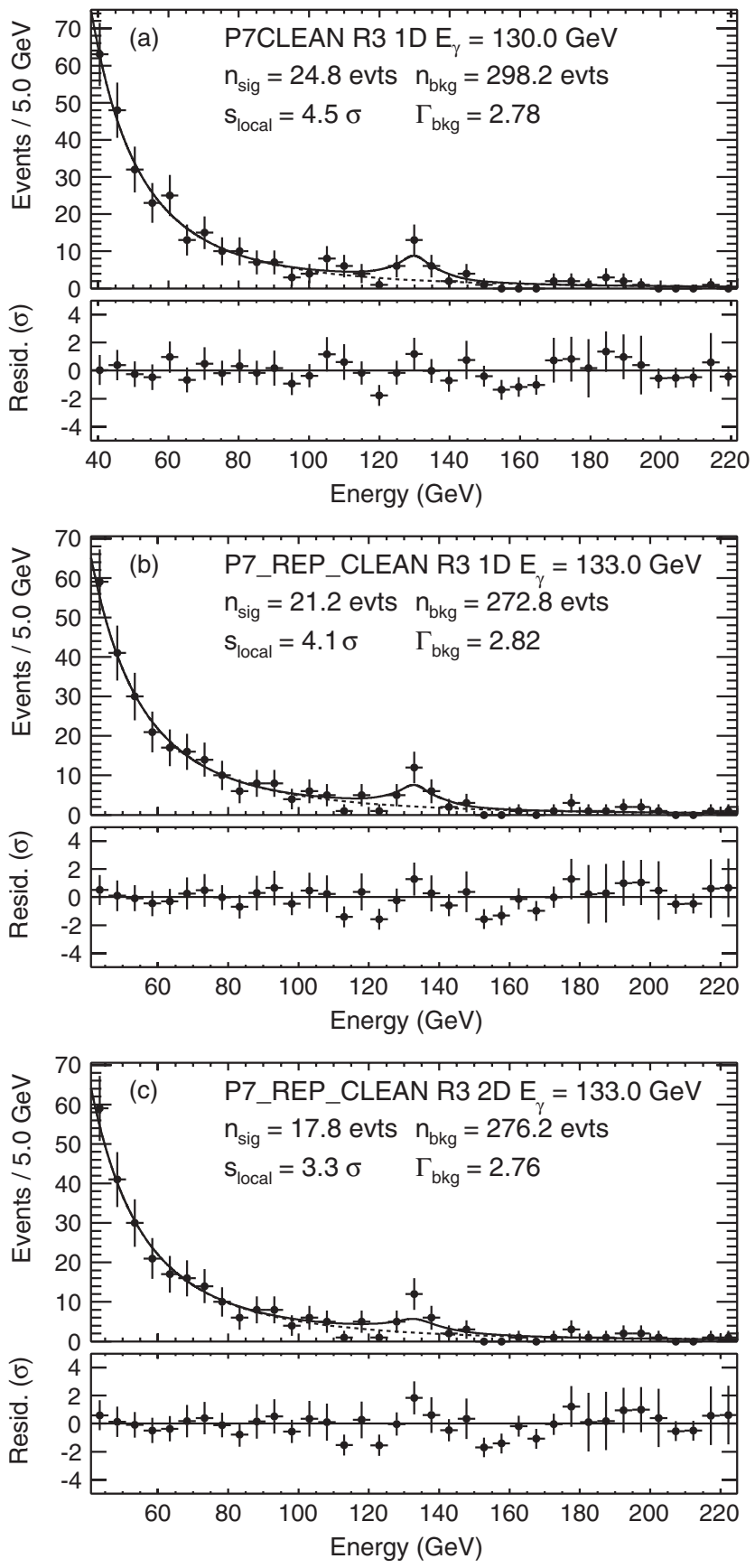

FIG. 12. Fits for a line near $130 \mathrm{GeV}$ in R3: (a) at $130 \mathrm{GeV}$ in the P7CLEAN data, using the 1D energy dispersion model (see Sec. IV); (b) at $133 \mathrm{GeV}$ in the P7REP_CLEAN data, again using the 1D model; (c) same as (b), but using the 2D energy dispersion model (see Sec. IV). The solid curve shows the average model weighted using the $P_{\mathrm{E}}$ distribution of the fitted events. Note that these fits were unbinned; the binning here is for visualization purposes, and also that the $x$ axis binning in (a) is offset by $3 \mathrm{GeV}$ relative to (b) and (c).
However, this masking only removes four events near $133 \mathrm{GeV}$ within $3^{\circ}$ of the Galactic center.

In order to better compare our results with the works referenced above, we fit the P7CLEAN (unreprocessed) data
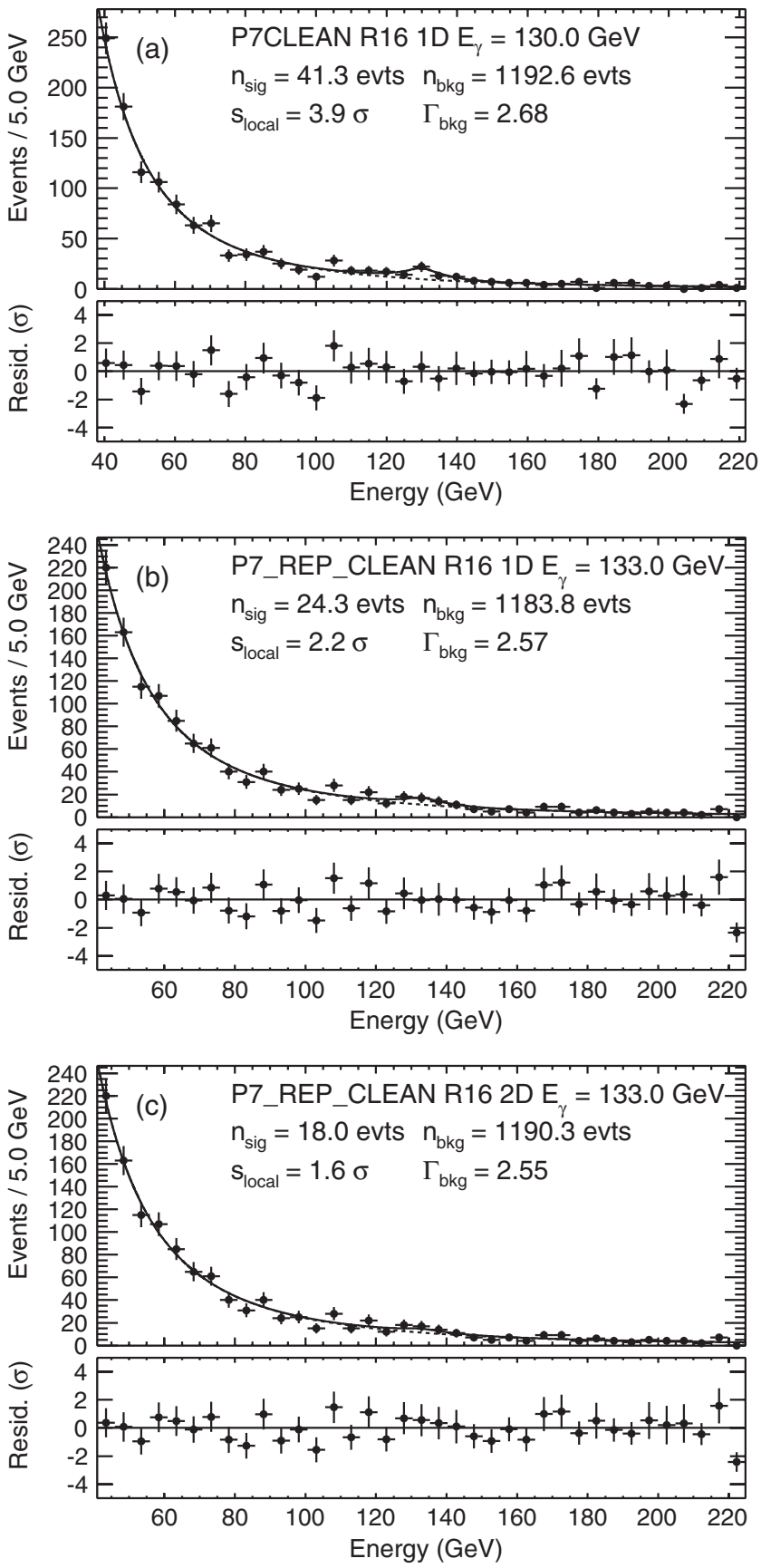

FIG. 13. Fit for a line near $130 \mathrm{GeV}$ in R16: (a) at $130 \mathrm{GeV}$ in the P7CLEAN data, using the 1D energy dispersion model (see Sec. IV); (b) at $133 \mathrm{GeV}$ in the P7REP_CLEAN data, again using the 1D model; (c) same as (b), but using the 2D energy dispersion model (see Sec. IV). The solid curve shows the average model weighted using the $P_{\mathrm{E}}$ distribution of the fitted events. Note that these fits were unbinned; the binning here is for visualization purposes, and also that the $x$ axis binning in (a) is offset by $3 \mathrm{GeV}$ relative to (b) and (c). 
in these ROIs with the 1D energy dispersion model that does not incorporate parametrization with $P_{E}$. The local significances for fits at $130 \mathrm{GeV}$ in $\mathrm{R} 3$ and $\mathrm{R} 16$ are $4.5 \sigma$ and $3.9 \sigma$, respectively [see Fig. 12(a) and 13(a)]. Since these fits were motivated by results outside of our search, we cannot estimate an effective trials factor, and we do not quote global significances for these fits.

Using the 1D energy dispersion model and fitting the P7REP_CLEAN at $133 \mathrm{GeV}$, we found local significances of $4.1 \sigma(\mathrm{R} 3)$ and $2.2 \sigma$ (R16) [see Fig. 12(b) and 13(b)]. It is worth noting that $70 \%-80 \%$ of events in the P7CLEAN data set are also in the P7REP_CLEAN data set, depending on energy. Therefore, small differences in $s_{\text {local }}$ are expected when evaluated with the P7CLEAN or P7REP_CLEAN data sets. We note in passing that the unmodeled slight smearing caused by the time-dependent shift in the absolute energy scale in the unreprocessed data degraded the energy resolution by less than $5 \%$ relative to the performance for P7REP_CLEAN.

Finally, when we used the 2D signal model, we found that the fits at $133 \mathrm{GeV}$ have local significances of $3.3 \sigma$ (R3) and 1.6 (R16) [see Fig. 12(c) and 13(c)].

Fitting the P7REP_CLEAN data set with the 2D energy dispersion model causes $s_{\text {local }}$ to decrease by $20 \%$ in R3 and $27 \%$ in R16 compared to fitting with the 1D model. Simulations predict that $s_{\text {local }}$ should increase, on average, by $15 \%$ in this case. A decrease by $20 \%$ or more occurred in $2 \%$ of the simulations. The decrease in significance with the 2D model implies that the clustering of events around the peak energy as a function of $P_{\mathrm{E}}$ in the flight data does not match variations in instrument performance well; this somewhat disfavors the interpretation of the $133 \mathrm{GeV}$ feature as a DM line.

To test if the feature persists with additional data, we also extracted a P7REP_CLEAN data set in R3 that includes data through December 12, 2012, and fit at $133 \mathrm{GeV}$. Figure 14 shows the fit results to this 4.4-year data set using both the 1D and 2D energy dispersion models. The local significance for the 1D energy dispersion model is $3.7 \sigma$, and it is $2.9 \sigma$ for the $2 \mathrm{D}$ energy dispersion model. The significance decreased by $\sim 10 \%$ with the 4.4-year data set relative to the 3.7-year data set. This is well within the expected statistical fluctuations for either the signal or the null hypothesis. Here again we do not quote global significances for these fits, as the studies were performed outside the context of our original search, and it is difficult to estimate a trials factor.

\section{B. Width of the feature near $133 \mathrm{GeV}$}

We note that the 2D model predicts a slightly broader energy distribution than the 1D model. As discussed in Sec. VA, the 2D model depends on the $P_{\mathrm{E}}$ distribution in the data. In fact, by inspection, the feature in the flight data appears to be narrower than both the 1D and 2D models, e.g., Figs. 12(b) and 12(c). To quantify this, we scaled the standard deviations of each of the three Gaussian functions that together are used to model the energy dispersion in each $P_{\mathrm{E}}$ bin in the 2D model by a common scale factor $\left(s_{\sigma}\right)$, while also scaling the means to preserve the overall shape of the model. This adds another degree of freedom to the fit with signal relative to the background-only fit $(\mathrm{n}=2)$. We then refit at $133 \mathrm{GeV}$ in the R3 ROI; the best-fit value was $s_{\sigma}=0.32_{-0.07}^{+0.11}$, as shown in Fig. 15 . The fit with $s_{\sigma}$ increases the TS by 9.4 relative to the fit without $s_{\sigma}$. In the case of scaling the 1D model, the fit returns $s_{\sigma}=0.44_{-0.13}^{+0.16}(\Delta T S=5.3)$. Prelaunch beam tests indicated that the uncertainty of the energy resolution is less than $10 \%$ of the measured resolution up to the maximum accessible beam energy of $280 \mathrm{GeV}$ [31]. Therefore, we conclude that the feature in the data is narrower than our expected energy resolution by a factor of $2-3$, and is inconsistent with the expected resolution at the $2 \sigma-3 \sigma$ level.

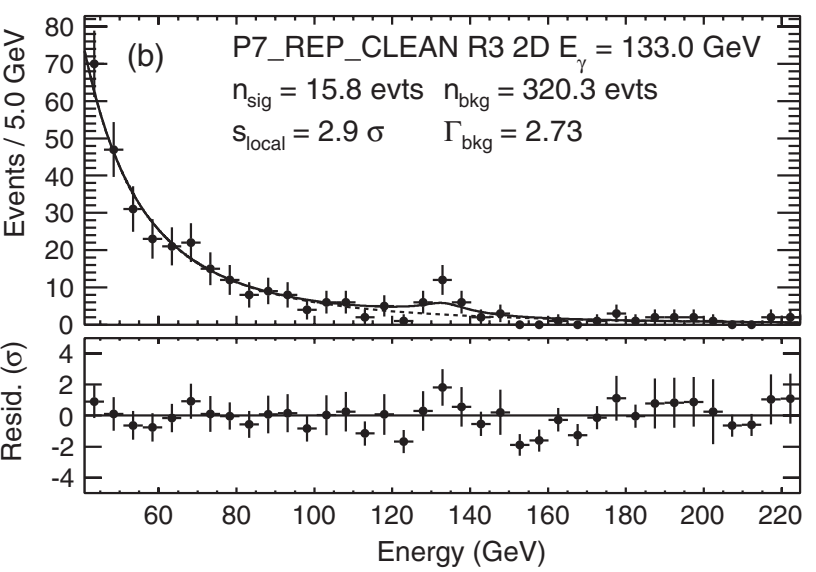

FIG. 14. Fit for a line-signal signal at $133 \mathrm{GeV}$ in R3 using a 4.4-year P7REP_CLEAN data set and (a) the 1D energy dispersion model; (b) the $2 \mathrm{D}$ energy dispersion model. The solid curve shows the average model weighted using the $P_{\mathrm{E}}$ distribution of the fitted events. Note that these fits were unbinned; the binning here is for visualization purposes. 


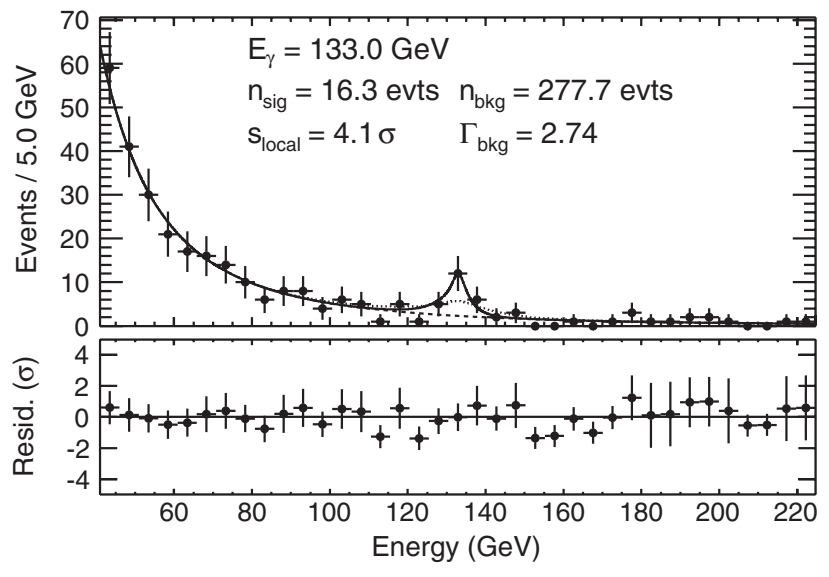

FIG. 15. Fit to a $\gamma$-ray line at $133 \mathrm{GeV}$ in the 3.7-year P7REP_CLEAN R3 data using the 2D model, including a scale factor for the width of the energy dispersion. The solid curves show the average model weighted using the $P_{\mathrm{E}}$ distribution of the fitted events. The best-fit width of the energy resolution was $s_{\sigma}=0.32_{-0.13}^{+0.30}(95 \%$ C.L. $)$ of that predicted from MC simulations. The dotted line shows the best-fit curve with $s_{\sigma}$ fixed to 1.0. Note that when $s_{\sigma}$ is allowed to vary, the signal model includes two more degrees of freedom than the null hypothesis, so $s_{\text {local }}$ is less than $\sqrt{T S}$. Also, note that these fits were unbinned; the binning here is for visualization purposes.

\section{C. $133 \mathrm{GeV}$ feature in the control regions}

We examine two control data sets that are expected to contain little or no DM. The first was the Limb data set (described in Sec. II A), while the second was a region centered on the Galactic plane but excluding the Galactic center, which we call the inverse ROI. The inverse ROI contains a variety of $\gamma$-ray sources but provides good statistics and a reasonable sample of the astrophysical backgrounds that we might expect from the Galactic center. See Table II for event selection details.

\section{The Earth Limb}

Figure 16 shows the fit using our 2D energy dispersion model (see Sec. IV) at $133 \mathrm{GeV}$ to the Limb data, which indicates a $2.0 \sigma$ excess. We calculated the fractional size of the signal using Eq. (14) to be $f(133 \mathrm{GeV})_{\text {Limb }}=$ $0.14 \pm 0.07$. The $\gamma$-ray spectrum of the Limb is expected to be featureless. Therefore, the appearance of a linelike feature in the Limb at the same energy as the feature seen in the Galactic center suggests that some of the $133 \mathrm{GeV}$ Galactic center feature may be due to a systematic effect. We do note that the fractional size of the feature in the Limb is smaller than observed in the smallest ROIs around the Galactic center: $f(133 \mathrm{GeV})_{\mathrm{R} 3}=0.61 \pm 0.19$. We also note that the significance of the feature in the Limb is somewhat reduced in the P7REP_CLEAN data set relative to the P7CLEAN data set, where $f(130 \mathrm{GeV})_{\text {Limb }}=0.18$.

The Limb is bright enough to be seen in the least stringent $\gamma$-ray selection, P7REP_TRANSIENT, which is

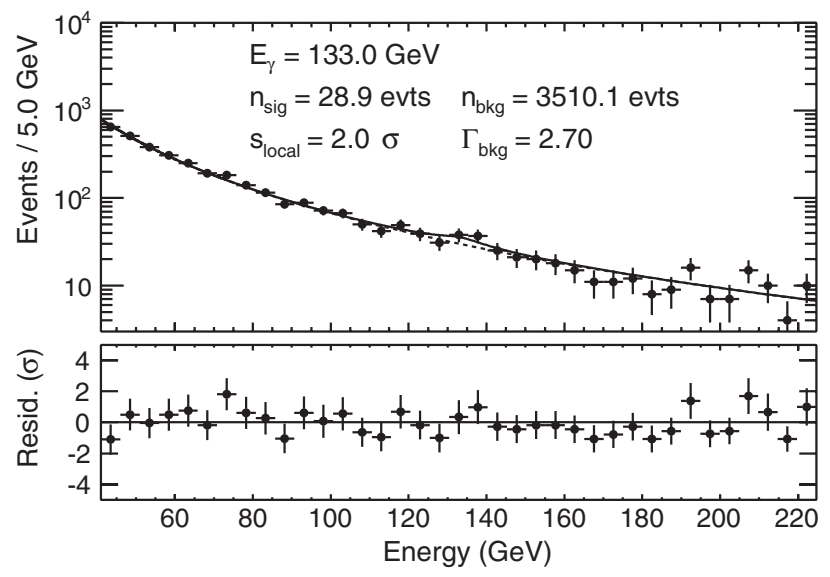

FIG. 16. Fit at the $133 \mathrm{GeV}$ line to the Limb data (P7REP_CLEAN) using the 2D energy dispersion model. The solid curve shows the average model weighted using the $P_{\mathrm{E}}$ distribution of the fitted events. Note that these fits were unbinned; the binning here is for visualization purposes.

meant to be used to study transient phenomena like $\gamma$-ray bursts. The P7REP_TRANSIENT event class has much higher rates of $\mathrm{CR}$ contamination than the P7REP_CLEAN class, $(\sim 10 \mathrm{~Hz}$ compared to $<0.1 \mathrm{~Hz})$, as it does not include some of the more stringent criteria needed to achieve the $\mathcal{O}\left(10^{5}\right) \mathrm{CR}$ rejection required for point source analysis. More details about the specific event selection criteria for the various event classes are available in Sec. 3.3 of Ref. [31]. We note for completeness that the P7REP_TRANSIENT Limb event sample does not show any feature at $133 \mathrm{GeV}$.

We have used the background subtraction technique described in Sec. 5.3.1 of Ref. [31] on both the P7REP_TRANSIENT and P7REP_CLEAN Limb samples to measure the $\gamma$-ray efficiency going from the P7REP_TRANSIENT to the P7REP_CLEAN selection as a function of energy. For this study, we used $111^{\circ}<\theta_{\mathrm{z}}<113^{\circ}$ for the signal region and $108.5^{\circ}<\theta_{z}<109.4805^{\circ}$ and $114.4701^{\circ}<\theta_{z}<$ $115.5^{\circ}$ for the background regions. The specific angles were chosen such that the signal and background regions contain the same solid angle. The $\theta_{\mathrm{z}}$ distributions for the signal and background regions, as well as the extracted efficiencies are shown in Fig. 17. The predicted efficiency based on the P7REP_TRANSIENT and P7REP_CLEAN IRFs and the observing profile for the Limb is also shown for comparison. While the predicted efficiency is smooth and featureless, the flight data suggest dips in efficiency above and below $133 \mathrm{GeV}$. The efficiency at $120 \mathrm{GeV}$ is $\sim 80 \%$ of the MC prediction, and $\sim 60 \%$ for the dip above $133 \mathrm{GeV}$.

We performed MC simulation studies with backgroundonly event samples, modified the exposure correction $\eta(E)$, based on the data-to-MC efficiency ratio from Fig. 17, and estimated the expected induced fractional signal when fitting with our 1D PDF. In all cases, the average induced fractional signal was less than $3 \%$. 

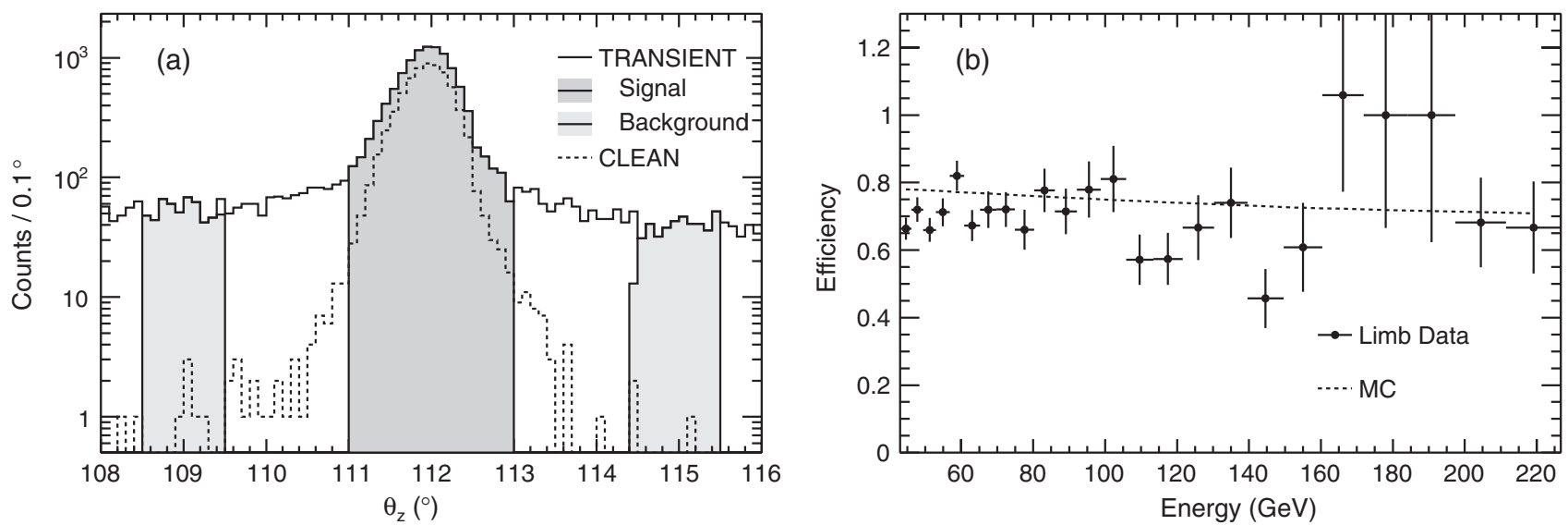

FIG. 17. Measurement of the P7REP_TRANSIENT-to-P7REP_CLEAN efficiency using the Limb control sample: (a) The distribution of $\theta_{z}$ for all events in the P7REP_TRANSIENT and P7REP_CLEAN Limb samples for $2.6 \mathrm{GeV}<E^{\prime}<541 \mathrm{GeV}$, including signal and background regions. (b) The P7REP_TRANSIENT-to-P7REP_CLEAN efficiency for Limb data and MC. MC has been weighted to have the same livetime distribution with $\theta$ as the Limb data.

However, we do not have adequate statistics to measure the efficiency in finer energy bins and cannot rule out narrower substructures contributing to an induced signal. Also, we observed in these simulations a systematic bias in the fitted
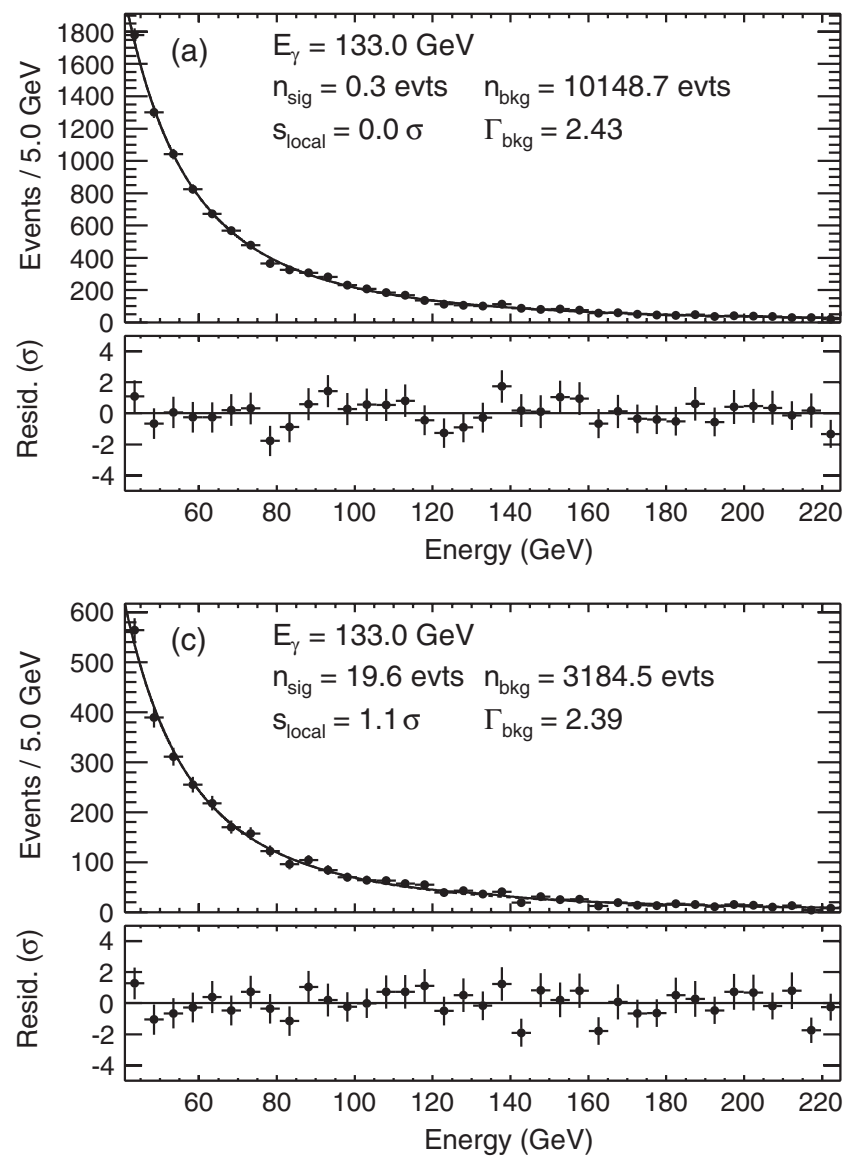

spectral index of $\delta \Gamma_{\mathrm{bkg}} \sim-0.05$, which in turn caused the fits to be more affected by upward statistical fluctuations near the fit energy and broadened the distribution of $n_{\text {sig }}$ by $\sim 20 \%-30 \%$.
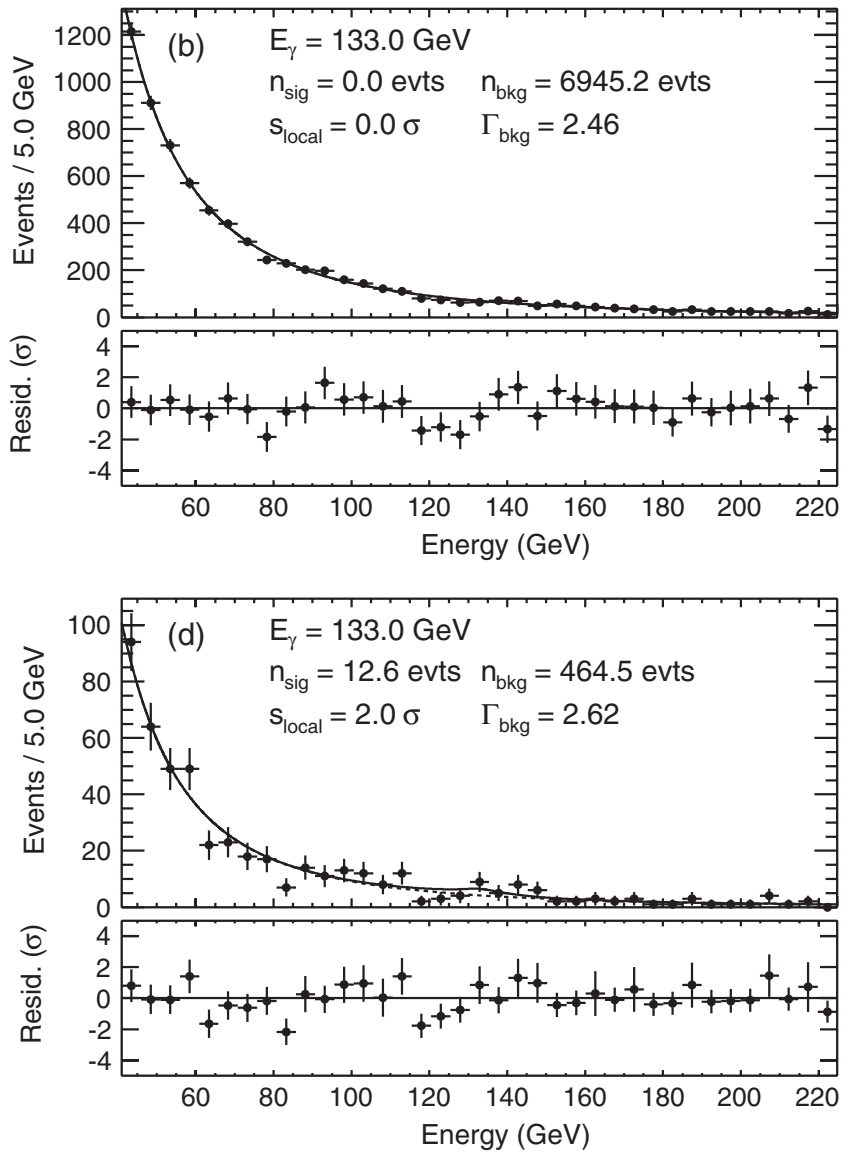

FIG. 18. Fits for a $133 \mathrm{GeV}$ line in inverse ROIs (P7REP_CLEAN) using the 2D energy dispersion model: (a) A, (b) B, (c) C, and (d) D. See text for definitions of regions $\mathrm{A}, \mathrm{B}$, and $\mathrm{C}$; region $\mathrm{D}$ is $|b|<10^{\circ}, 90^{\circ}<l<110^{\circ}$. The solid curves show the average model weighted using the $P_{\mathrm{E}}$ distribution of the fitted events. Note that these fits were unbinned; the binning here is for visualization purposes. 
Therefore, although suggestive, we do not believe that the measured variations in $\gamma$-ray efficiency provide a complete explanation for the observed feature of $f(133 \mathrm{GeV})_{\text {Limb }}=0.14$, which is well outside the range of induced signals seen in the Limb, which are typically less than $f=0.05$; see Sec. D 7 a. The potential origin of the features observed in the transient-to-clean efficiency observed in the Limb data is discussed further in Sec. VIII D.

In Fig. 17(a), it is clear that in the P7REP_CLEAN selection the $\theta_{\mathrm{z}}$ background regions contain very few events; in fact, the exposure for the Limb sample is over 400 times smaller than for the Celestial sample; therefore, the expected cross contamination of the Limb sample from a signal of $\sim 25$ events at the Galactic center would be less than a single event.

\section{The inverse ROI}

As a further control study, we also searched for features elsewhere along the Galactic plane. We define the inverse ROI A to be events with $|b|<10^{\circ}$, excluding a $20^{\circ} \times 20^{\circ}$ square in the Galactic center in the Celestial data set. In addition to $\mathrm{A}$, we also examined inverse ROIs $\mathrm{B}$ and $\mathrm{C}$, which are subsets of inverse ROI A with $|b|>1^{\circ}$ and $|b|<1^{\circ}$, respectively. Figure 18 shows the results of fits for lines at $133 \mathrm{GeV}$ in the three inverse ROI regions. Regions A, B, and C show no indication of a linelike feature at $133 \mathrm{GeV}$ with $s_{\text {local }}>$ $1.1 \sigma$. We also scanned using $20^{\circ} \times 20^{\circ}$ ROIs along the Galactic plane, resulting in 17 independent fits. Figure 18(d) shows the results from the fit at $133 \mathrm{GeV}$ with the greatest statistical significance, where $s_{\text {local }}=$ $2.0 \sigma$. Thus, we find no clear indication for a $133 \mathrm{GeV}$ line feature in these inverse ROI control data sets.

\section{Examination of the events contributing to the $133 \mathrm{GeV}$ feature}

We have examined many aspects of the events contributing to the $133 \mathrm{GeV}$ feature and compared them to events at nearby energies as well as with MC simulations. Within the limited statistics available, the events contributing to the $133 \mathrm{GeV}$ feature exhibit few particularly striking characteristics. The two most notable features are as follows:

(1) The consistency between the reconstructed direction as estimated by the tracker and the primary axis of the energy deposition in the calorimeter is somewhat worse in the flight data than in the MC simulations (Fig. 19). The disagreement was even greater before reprocessing the data with updated calorimeter calibration constants. This disagreement is seen in several quantities that contribute strongly to determining $P_{\mathrm{E}}$, so it is unsurprising that $P_{\mathrm{E}}$ tends to have slightly lower values in the flight data, or that the data-MC agreement of the $P_{\mathrm{E}}$ distribution has improved with the reprocessed data (see Fig. 5). We also note that, with the available statistics, the flight data from R16 are consistent with the distribution from the entire sky.

(2) The $\theta$ distribution of the events contributing to the $133 \mathrm{GeV}$ feature is marginally different statistically than for events at other energies and the MC predictions. This is discussed in more detail in Sec. VIII E.

\section{E. $\theta$ dependence of the $133 \mathrm{GeV}$ feature}

Several authors have reported a $\theta$ dependence of the prominence of the spectral feature in both the Limb and Galactic center data sets, which is unexpected [27-29]. Our results are broadly consistent with those previously
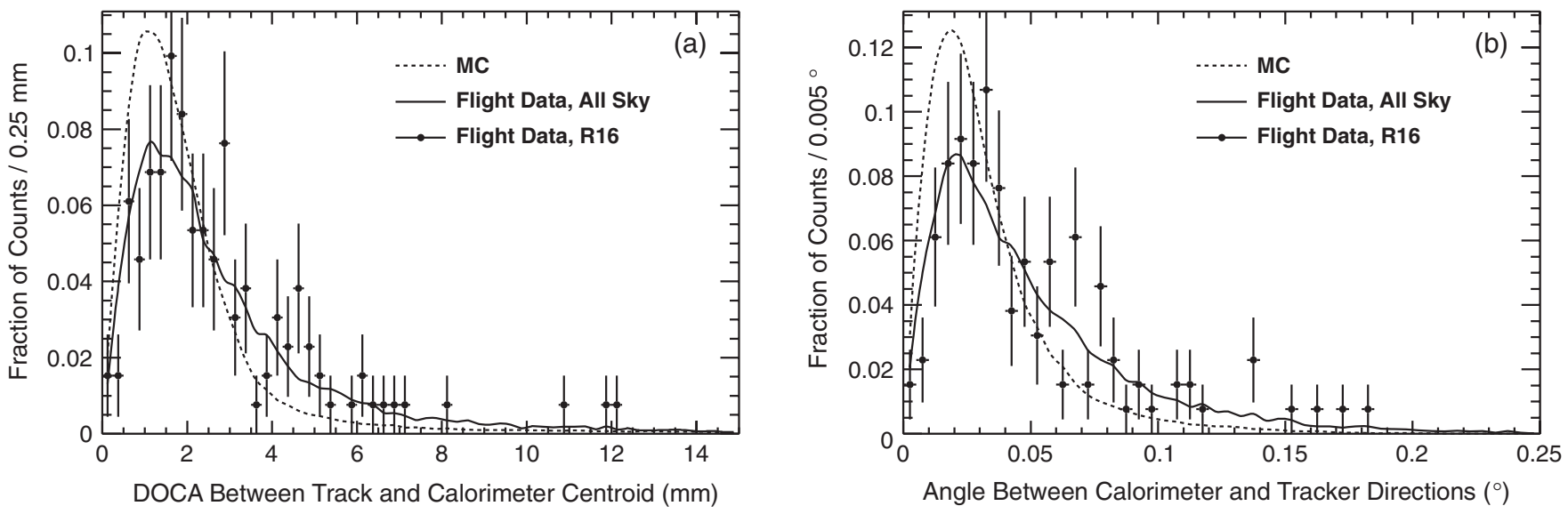

FIG. 19. Two measures of the calorimeter-tracker event direction agreement for events with $125 \mathrm{GeV}<\mathrm{E}^{\prime}<145 \mathrm{GeV}$ : (a) The distance of closest approach (DOCA) between the extrapolation of the tracker direction and the centroid of the calorimeter energy deposition. (b) The angle between the tracker direction and the primary axis of the energy deposition in the calorimeter. In both curves, the MC has been weighted to have the same livetime distribution with $\theta$ as the all-sky data, and the small $(<0.005)$ error bars on the all-sky sample have been suppressed for visual clarity. 

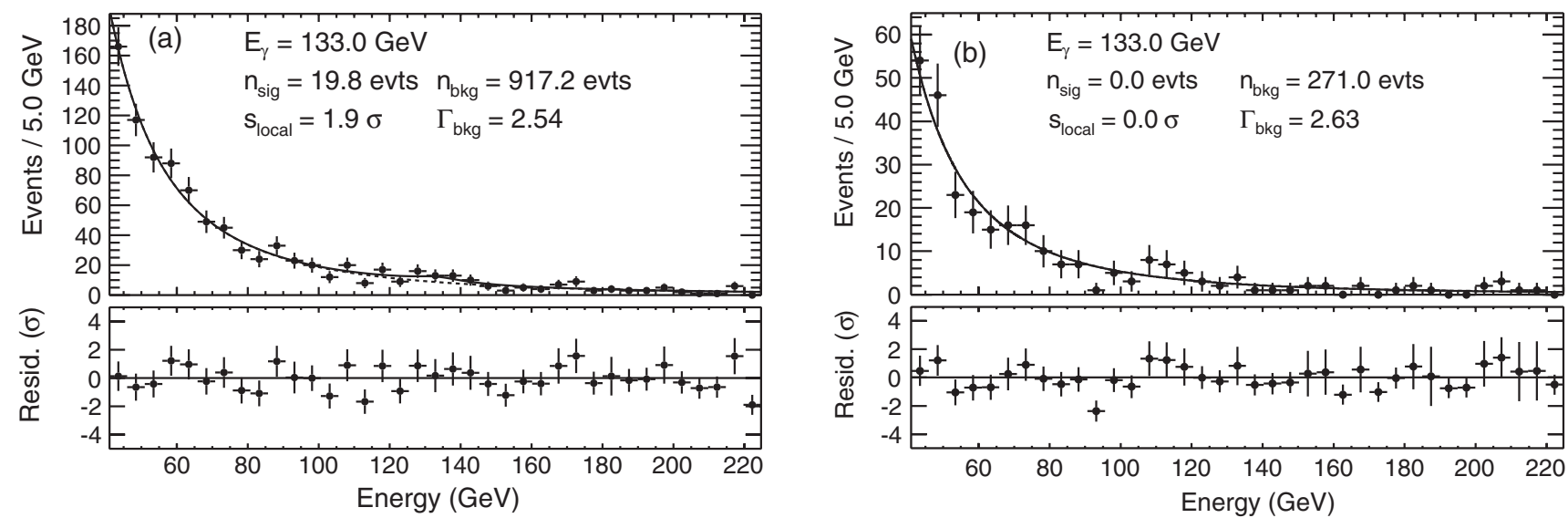

FIG. 20. Fit at the $133 \mathrm{GeV}$ line in R16 (P7REP_CLEAN) using the $2 \mathrm{D}$ energy dispersion model (a) for events with $\theta<50^{\circ}$, and (b) for events with $\theta>50^{\circ}$. The solid curves show the average model weighted using the $P_{\mathrm{E}}$ distribution of the fitted events. Note that these fits were unbinned; the binning here is for visualization purposes.

reported; the feature appears with a larger statistical significance in data sets of events with smaller incident angles. To study this near the Galactic center, we fit for a line at $133 \mathrm{GeV}$ in $\mathrm{R} 16$ in two $\theta$ ranges: $\theta<50^{\circ}$ and $\theta>50^{\circ}$. This ROI was chosen for this study to have enough events to separately consider both $\theta$ ranges. Figure 20 shows the fit results in both $\theta$ ranges. There is no evidence of any feature at $133 \mathrm{GeV}$ from events with $\theta>50^{\circ}$, while the fit using events with $\theta<50^{\circ}$ indicates a feature at $133 \mathrm{GeV}$ with $s_{\text {local }}=1.9 \sigma$. Though there are fewer events with $\theta>50^{\circ}$, the observed fractional size from the events with $\theta<50^{\circ}, \quad f\left(E_{\gamma}=133 \mathrm{GeV}\right)_{\theta<50^{\circ}}=0.18$ should scale to produce a feature with $1.0 \sigma$ given the number of events with $\theta>50^{\circ}$; see Eq. (14).

Similarly, we split the Limb data set into the same ranges of $\theta$. For events with $\theta<50^{\circ}$, the significance is $s_{\text {local }}=$ $2.6 \sigma$, while for the events with $\theta>50^{\circ}$, the significance is $s_{\text {local }}=0.0 \sigma$ (see Fig. 21).

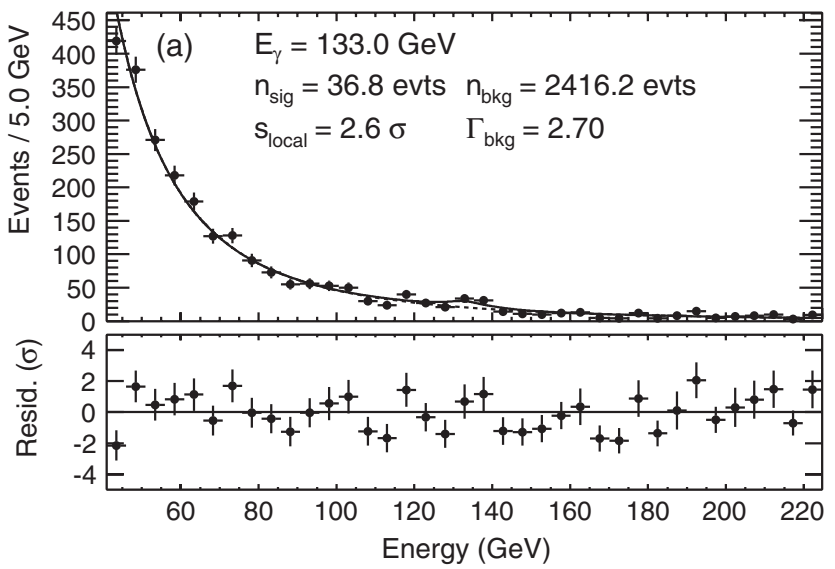

\section{DISCUSSION AND SUMMARY}

We have performed a search for $\gamma$-ray spectral lines from $5-300 \mathrm{GeV}$ in five ROIs defined a priori to optimize sensitivity for various DM density profiles. This search was performed using 3.7 years of data that have been reprocessed using updated calorimeter calibration constants and the 2D energy dispersion model that includes information about the event-by-event energy reconstruction quality.

We found no globally significant spectral line signals, and we present flux upper limits for monochromatic sources (see Tables VII, VIII, IX, and X). For a particular DM density profile for the Milky Way, the flux upper limits can be translated to annihilation-cross-section upper limits or decay-lifetime lower limits. Figure 10 shows the 95\% CL upper limits on $\langle\sigma v\rangle_{\gamma \gamma}$ for the contracted NFW (R3), Einasto (R16), NFW (R41), and isothermal (R90) DM density profiles for the ROIs that provide the best

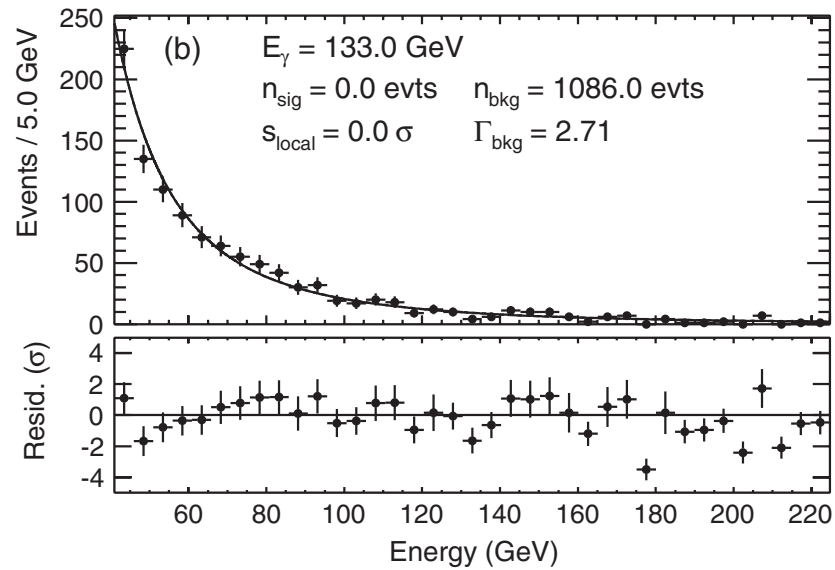

FIG. 21. Fit at the $133 \mathrm{GeV}$ line in the Limb (P7REP_CLEAN) using the $2 \mathrm{D}$ energy dispersion model (a) for events with $\theta<50^{\circ}$, and (b) for events with $\theta>50^{\circ}$. The solid curves show the average model weighted using the $P_{\mathrm{E}}$ distribution of the fitted events. Note that these fits were unbinned; the binning here is for visualization purposes. 
sensitivity. Also shown are the $95 \%$ C.L. lower limits on the decay lifetime $\left(\tau_{\nu \gamma}\right)$ for the R180 ROI, assuming a NFW profile. The cross section upper limits have been improved in some cases by a factor of several relative to the LAT Collaboration two-year limits [18] and represent an extension of the search range from 7-200 GeV to 5-300 GeV.

Our new velocity-averaged cross section limits lie in the range $\langle\sigma v\rangle_{\gamma \gamma} \sim 10^{-29}-10^{-27} \mathrm{~cm}^{3} \mathrm{~s}^{-1}$, with the precise limit depending on the WIMP mass and the DM density profile assumed for the Milky Way; cuspier profiles and lower masses are constrained more strongly. The limits are a factor of $\sim 5-5000$ times below the canonical thermal relic cross section of $\langle\sigma v\rangle_{\mathrm{WIMP}} \sim 3 \times 10^{-26} \mathrm{~cm}^{3} \mathrm{~s}^{-1}$ and therefore strongly constrain models in which DM particles can annihilate to standard model particles through treelevel diagrams. However, since DM is constrained to be electrically neutral to a very good approximation, WIMP interactions in most models produce monochromatic photons only through higher-order processes, the cross sections of which are typically suppressed by 3 or more orders of magnitude. This means that our limits do not disfavor the WIMP hypothesis in general.

Our two most significant fits occurred at $6 \mathrm{GeV}$ in R180 and at $133 \mathrm{GeV}$ in R3. While the fit at $6 \mathrm{GeV}$ in R180 has a relatively large $T S$ value, the signal fraction (1\%) was similar to the expected systematic uncertainty of $f \sim$ 0.008 for R180 at that energy.

Reports of a linelike feature in the Galactic center using the public data have appeared in the literature [19,20,22]. The authors calculated the flux of the source producing the linelike feature to be $\sim 2 \times 10^{-10} \mathrm{~cm}^{-2} \mathrm{~s}^{-1}$, which is not ruled out by our $95 \%$ C.L. $\Phi_{\gamma \gamma}$ limits in R3 $(3.4 \times$ $10^{-10} \mathrm{~cm}^{-2} \mathrm{~s}^{-1}$ for $E_{\gamma}=135 \mathrm{GeV}$, see Table X). Additionally, these reported fluxes are similar to the mean value obtained from our fit at $133 \mathrm{GeV}$ in R3 of $\Phi_{\gamma \gamma}^{R 3}\left(E_{\gamma}=133 \mathrm{GeV}\right)=1.9 \times 10^{-10} \mathrm{~cm}^{-2} \mathrm{~s}^{-1}$.

The fit at $133 \mathrm{GeV}$ in R3 yields $s_{\text {local }}=3.3 \sigma$ with $f(133 \mathrm{GeV})_{R 3}=0.61$, which is larger than any of the systematic effects summarized in Sec. VI (see Table IV) and is larger than the feature seen at $133 \mathrm{GeV}$ in the Limb: $f(133 \mathrm{GeV})_{\text {Limb }}=0.14$. Also, if the feature is due to an instrumental effect, one would have expected it to appear in the spectra of $\gamma$ rays from the inverse ROI, which it does not. Therefore, the $133 \mathrm{GeV}$ feature in R3 cannot be entirely explained in terms of known systematic effects. However, as discussed in Sec. VIII, the $133 \mathrm{GeV}$ feature does have certain characteristics that disfavor interpreting it as a DM signal. The fit significance reduces when using the $2 \mathrm{D}$ energy dispersion model, making the global significance of the feature $s_{\text {global }}=1.5 \sigma$. This decrease in significance is in large part due to the $133 \mathrm{GeV}$ feature being much narrower than the LAT energy resolution, and not being present in events with $\theta>50^{\circ}$. More data and study are needed to clarify the origin of this feature.
Two ongoing developments will help to resolve the question of the origin of the $133 \mathrm{GeV}$ feature and also benefit future line searches:

(1) Beginning October 2012, the LAT started collecting more data from the Limb through weekly two-orbit pointed observations at the orbital pole. This change alone should increase the available Limb data set by $\sim 40 \%$ over the next year and will decrease the current statistical limitations at high energies $(>100 \mathrm{GeV})$ in the Limb. Additional Limb data can also be collected during "target of opportunity" pointed observations if Limb tracing is implemented while the target is occulted by the Earth. These data will help to constrain the uncertainties from narrow features in the effective area, which are among the dominant sources of potential systematic uncertainties that may induce a false signal.

(2) Almost every aspect of the LAT event reconstruction and selection algorithms has been rewritten in the new and upcoming PASS 8 versions. Expected improvements most relevant to a line search are an increased effective area at all energies and an improved energy resolution, particularly at higher energies [43]. Furthermore, aside from any performance improvements, given the scope of the changes in PASS 8, systematic biases associated with the event reconstruction and selection in PASS 8-based analyses are likely to be uncorrelated with similar biases in PASS 7-based analyses, which will help clarify if the feature at $133 \mathrm{GeV}$ is a systematically induced artifact.

\section{ACKNOWLEDGMENTS}

The Fermi-LAT Collaboration acknowledges generous ongoing support from a number of agencies and institutes that have supported both the development and the operation of the LAT as well as scientific data analysis. These include the National Aeronautics and Space Administration and the Department of Energy in the United States, the Commissariat à l'Energie Atomique and the Centre National de la Recherche Scientifique/ Institut National de Physique Nucléaire et de Physique des Particules in France, the Agenzia Spaziale Italiana and the Istituto Nazionale di Fisica Nucleare in Italy, the Ministry of Education, Culture, Sports, Science and Technology (MEXT), High Energy Accelerator Research Organization (KEK), and Japan Aerospace Exploration Agency (JAXA) in Japan, and the K. A. Wallenberg Foundation, the Swedish Research Council, and the Swedish National Space Board in Sweden. Additional support for science analysis during the operations phase is gratefully acknowledged from the Istituto Nazionale di Astrofisica in Italy and the Centre National d'Études Spatiales in France. We would also like to thank Christoph Weniger for providing the limit values used in 
Fig. 10. J. Conrad is a Royal Swedish Academy of Sciences Research Fellow, funded by a grant from the K. A. Wallenberg Foundation. A. B. Hill was funded by a Marie Curie IOF, FP7/2007-2013 under Grant No. 275861. E. Troja is a NASA Postdoctoral Program Fellow.

\section{APPENDIX A: PASS 7 DATA REPROCESSING}

In 2012, the LAT Collaboration reprocessed all of the data from the mission to date with updated calibrations for the instrument subsystems, but with the same reconstruction and event-level analysis algorithms as the previously released PASS 7 data.

The primary goal of this reprocessing was to incorporate improved calibrations of the measurement of the light asymmetry between the ends of the calorimeter crystals. This asymmetry is used to derive position information that is critical to measuring the centroid and axis of the electromagnetic shower in the calorimeter. See Fig. 12 of Ref. [31] for an illustration of the calorimeter shower, including the centroid and axis. Above a few GeV, both the centroid and axis of the electromagnetic shower are useful in constraining the event reconstruction in the tracker, which would otherwise be degraded because of the increased event complexity at these high energies caused by the backscattering of particles from the calorimeter back into the tracker. Specifically, using the calorimeter shower centroid as an additional constraint on the event direction can significantly reduce the tails of the PSF. Furthermore, the consistency between the tracker direction solution and both the calorimeter shower axis and the centroid are powerful discriminators between $\gamma$ rays and CR background events (see Sec. 3.4.3 of Ref. [31], in particular items 3 and 4 in the bulleted list).

The updated calibrations also corrected for a small $(\sim 1 \%$ per year) expected degradation in the light yield of the calorimeter crystals that had been measured in the flight data (see Fig. 73 of Ref. [31] and the accompanying text). Consequently, the absolute energy scale has shifted up by a few percent in an energy- and time-dependent way. This has caused the feature reported at $130 \mathrm{GeV}$ with PASS 7 data to shift to $133 \mathrm{GeV}$ with PASS 7REP data.

\section{APPENDIX B: REGION OF INTEREST OPTIMIZATION}

Following Bringmann et al. [20] and Weniger [19], we have adopted a method for defining optimized ROIs by comparing the signal $\gamma$ rays expected from WIMP annihilation or decay, assuming a specific density profile, to the background $\gamma$ rays expected from astrophysical processes. Unlike Bringmann et al. [20] and Weniger [19], who estimated the expected $\gamma$-ray background from the LAT $\gamma$-ray data, we used the gtobssim ScienceTool to simulate two years of LAT observation of the expected backgrounds based on the standard LAT models of diffuse emission from the Galaxy and isotropic emission. The differential $\gamma$-ray flux from the annihilation of selfconjugate WIMPs is

$$
\left(\frac{d \Phi_{\gamma}}{d E d \Omega}\right)_{\mathrm{ann}}=\frac{1}{8 \pi} \frac{\langle\sigma v\rangle}{m_{\chi}^{2}}\left(\frac{d N_{\gamma}}{d E}\right)_{\mathrm{ann}} \frac{d J_{\mathrm{ann}}}{d \Omega},
$$

with

$$
\frac{d J_{\mathrm{ann}}}{d \Omega}=\int d s \rho(r)^{2},
$$

where the integration is performed over the square of the DM mass density $(\rho)$ along the line of sight, $\langle\sigma v\rangle$ is the velocity-averaged annihilation cross section, $m_{\chi}$ is the WIMP mass, and $d N_{\gamma} / d E$ is the differential $\gamma$-ray yield per annihilation. The differential $\gamma$-ray flux from WIMP decays is

$$
\left(\frac{d \Phi_{\gamma}}{d E d \Omega}\right)_{\text {decay }}=\frac{1}{4 \pi} \frac{1}{\tau} \frac{1}{m_{\chi}}\left(\frac{d N_{\gamma}}{d E}\right)_{\text {decay }} \frac{d J_{\text {decay }}}{d \Omega}
$$

with

$$
\frac{d J_{\text {decay }}}{d \Omega}=\int d s \rho(r)
$$

where $\tau$ is the DM particle lifetime. The total signal from $\mathrm{DM}$ is given by an integration over the ROI,

$$
\left(\frac{d \Phi_{\gamma}}{d E}\right)_{\mathrm{ann}}=\frac{1}{8 \pi} \frac{\langle\sigma v\rangle}{m_{\chi}^{2}}\left(\frac{d N_{\gamma}}{d E}\right)_{\mathrm{ann}} \int^{\mathrm{ROI}} \frac{d J_{\mathrm{ann}}}{d \Omega} d \Omega
$$

for annihilations, and

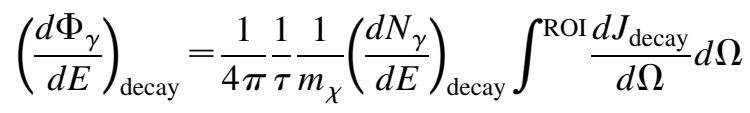

for decays. The integral $\int^{\mathrm{ROI}} \frac{d J}{d \Omega} d \Omega$ is commonly referred to as the "J factor" and represents the astrophysical component of the DM flux calculation.

We define our ROI as a circular region of radius $R_{\mathrm{GC}}$ centered on the galactic center. We additionally mask a rectangular region along the Galactic plane with $|b|<\Delta b$ and $|l|>\Delta l$. This ROI definition excludes emission from the off-center Galactic plane, where the astrophysical background is largest and the expected DM contribution is relatively small.

To remove the Galactic plane, we set $\Delta b=5^{\circ}$. We then optimize the remaining ROI parameters $\left(R_{\mathrm{GC}}\right.$ and $\left.\Delta l\right)$ for each of our four models of the MW DM halo. Note that because our fits are background dominated at 
all but the highest energies, the optimization is insensitive to any prefactor in the signal model. Specifically, we maximize the signal-to-noise ratio $(S / N)$ defined for a given ROI as

$$
S / N_{\mathrm{ROI}}=\frac{\int^{\mathrm{ROI}} \int^{\mathrm{FOV}} S(\hat{p}) \mathcal{E}(\hat{p}) d \Omega_{\hat{v}} d \Omega}{\sqrt{\int^{\mathrm{ROI}} \int^{\mathrm{FOV}} B(\hat{p}) \mathcal{E}(\hat{p}) d \Omega_{\hat{v}} d \Omega}},
$$

where $\mathcal{E}$ is the exposure, and $S$ and $B$ are the intensities of $\gamma$ rays in the direction $\hat{p}$ from DM and diffuse backgrounds, respectively. We model the spatial distribution of the diffuse background by integrating the $\gamma$ rays between 10 and $100 \mathrm{GeV}$ from a simulation of two years of LAT observations using gtobssim and the recommended templates for the isotropic and Galactic diffuse emission. The optimization results do not change significantly when only background events with energies from 10 to $30 \mathrm{GeV}$ or 30 to $100 \mathrm{GeV}$ are used. Therefore, this method appears not to have a strong dependence on the energy range (above $10 \mathrm{GeV}$ ).

The value of $\Delta l=6^{\circ}$ is close to optimal for all but the contracted NFW profile, which is not affected by the Galactic plane mask. In the case of annihilating DM, there is a strong dependence of $R_{\mathrm{GC}}$ on the shape of the inner profile. The smallest ROIs are preferred for the profiles with the largest central densities. Figure 22 shows $S / N_{\text {ROI }}$ normalized to its maximum value as a function of the ROI parameters $\Delta l$ and $R_{\mathrm{GC}}$ evaluated from $0.5^{\circ}-30^{\circ}$ and $0.5^{\circ}-180^{\circ}$, respectively. Note that in Fig. 22, $R_{\mathrm{GC}}$ (a) and $\Delta l$ (b) have been fixed at their optimal values.

For the contracted NFW profile, the optimal $R_{\mathrm{GC}}$ is found at the smallest radius considered $\left(0.5^{\circ}\right)$, which is at the characteristic scale of the LAT PSF at $1 \mathrm{GeV}$. Optimization of the ROI with $R_{\mathrm{GC}} \lesssim 0.5^{\circ}$ would require convolving the DM signal profile with the LAT PSF and require a different, more complicated analysis than the one presented in this paper. In the case of decaying DM, the optimal ROI parameters are nearly independent of the shape of the DM distribution, preferring a large optimal $R_{\mathrm{GC}}$ for all four profiles studied.

We define a set of five ROIs with a fixed Galactic plane mask $\left(\Delta l=6^{\circ}\right.$ and $\left.\Delta b=5^{\circ}\right)$ and the following values of $R_{\mathrm{GC}}: 3^{\circ}(\mathrm{R} 3), 16^{\circ}$ (R16), $41^{\circ}$ (R41), 90 (R90), and $180^{\circ}$ (R180). We use the smallest ROI (R3) to search for a signal compatible with the contracted NFW profile. In this instance, the ROI size of $3^{\circ}$ was intentionally chosen to be larger than the region with the best $S / N$. For the contracted NFW profile, the $S / N$ of $\mathrm{R} 3$ is reduced by $40 \%$ when compared to the smallest circular region in our optimization scan $\left(R_{\mathrm{GC}}=0.5^{\circ}\right)$. On the other hand, by using a larger search region, the analysis is less dependent on the LAT PSF. Additionally, we limit the search in R3 to spectral lines above $30 \mathrm{GeV}$. At these high energies, emission from known $\gamma$-ray sources is at least an order of magnitude dimmer than the Galactic diffuse emission integrated over R3. Thus, we also avoid complications from point sources and no longer need to apply a source mask (see Table II). This allows us to use all of the events in this already small, event-limited ROI.

The ROIs R16, R41, and R90 were chosen to optimize the sensitivity to annihilating DM assuming the Einasto, NFW, and isothermal halo models, respectively. Finally, we chose a large ROI (R180), which is close to optimal for decaying DM models. R180 is also similar

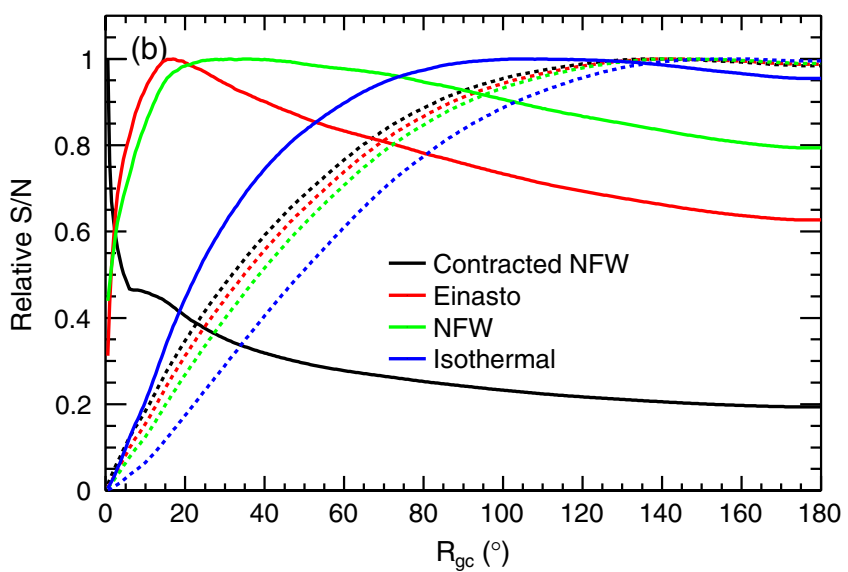

FIG. 22 (color online). Evaluation of the optimal values of $\Delta l$ and $R_{\mathrm{GC}}$. The plots show $S / N$ as a function of (a) $\Delta l$ and (b) $R_{\mathrm{GC}}$ normalized to the maximum for different DM density profiles (see legend) for DM annihilations (solid lines) and decays (dashed lines). For (a), $\Delta b$ has been fixed to $5^{\circ}$, and $R_{\mathrm{GC}}$ has been fixed to the optimal value for the associated DM density profile (see text). The vertical dashed line shows the fixed value of $\Delta l$ that was chosen for all optimized ROIs. (We do not show the case for DM annihilation with a contracted NFW profile, for which the optimal ROI is smaller than our Galactic plane mask.) For (b), $\Delta b$ and $\Delta l$ have been fixed to $5^{\circ}$ and $6^{\circ}$, respectively. 
to the ROI used in the previous LAT Collaboration line search [18]. ${ }^{9}$

\section{APPENDIX C: LIKELIHOOD FORMALISM}

Many Fermi-LAT analyses, and many of the ScienceTools, follow the likelihood formalism of Mattox et al. [44] to test hypotheses about the spatial and spectral distribution of observed $\gamma$ rays. In this paper, we have made several assumptions and approximations to derive the parametrized likelihood function that we used for our analysis. In this appendix, we discuss those assumptions and approximations.

\section{General formalism}

We test the hypothesis that the distribution of measured energies $E^{\prime}$, directions $\hat{p}^{\prime}$, arrival times $t$, and other observable parameters $\vec{s}$ comes from a model of the total source fluxes, $S(E, \hat{p})$, where $E$ and $\hat{p}$ are the true energies and directions of the incident $\gamma$ rays. This testing requires a representation of the response of the LAT: $R\left(E^{\prime}, \hat{p}^{\prime} ; E, \hat{p}, \vec{s}, t\right)$. Note that $\vec{s}$ represents event parameters (e.g., $P_{\mathrm{E}}$, or the tracker layer at which the $\gamma$-ray converted), as well as the event selection (e.g., P7REP_CLEAN).

In practice, we parametrize the instrument response as a function of the $\gamma$-ray direction in the LAT reference frame, $\hat{v}$. Furthermore, since the LAT performance has changed little over the course of the mission, we use a single representation for the entire mission. Therefore, we express the instrument response as $R\left(E^{\prime}, \hat{p}^{\prime} ; E, \hat{v}(t ; \hat{p}), \vec{s}\right)$, where we have explicitly indicated that the reference frame of the LAT rotates with respect to the celestial frame by writing the time dependence of $\hat{v}$. From here on, we suppress the dependencies of $\hat{v}$ on $t$ and $\hat{p}$.

The parametrization provided with the ScienceTools factors the instrument response into three parts:

(1) The effective collecting area for $\gamma$ rays for a given $E, \hat{v}$ and $\vec{s}: A_{\text {eff }}(E, \hat{v}, \vec{s})$.

(2) The PSF, i.e., the probability density to reconstruct an incident direction $\hat{p}^{\prime}$, for a given $E, \hat{p}, \hat{v}$ and $\vec{s}$ : $P\left(\hat{p}^{\prime} ; \hat{p}, E, \hat{v}, \vec{s}\right)$.

(3) The energy dispersion, i.e., the probability density to measure $E^{\prime}$, for a given $E, \hat{v}$ and $\vec{s}: D\left(E^{\prime} ; E, \hat{v}, \vec{s}\right)$.

Note that by factoring the instrument response in this way, we implicitly assumed that the spatial dispersion and energy dispersion are uncorrelated for given $E, \hat{v}$ and $\vec{s}$.

We can obtain the predicted $\gamma$-ray distribution, $M\left(E^{\prime}, \hat{p}^{\prime} ; \vec{s}\right)$, by convolving the source flux models with the IRFs and integrating over the spatial, energy, and time ranges of interest:

\footnotetext{
${ }^{9}$ The previous LAT Collaboration line search used a Galactic plane mask with $\Delta l=10^{\circ}$ and $\Delta b=10^{\circ}$.
}

$$
\begin{aligned}
M\left(E^{\prime}, \hat{p}^{\prime} ; \vec{s}\right)= & \iiint S(E, \hat{p}) A_{\mathrm{eff}}(E, \hat{v}, \vec{s}) P\left(\hat{p}^{\prime} ; \hat{p}, E, \hat{v}, \vec{s}\right) \\
& \times D\left(E^{\prime} ; E, \hat{v}, \vec{s}\right) d \Omega d E d t .
\end{aligned}
$$

We make a few approximations to simplify this expression, and to improve the computational efficiency of the analysis:

(1) The performance variation as a function of the angle with respect to the boresight $(\theta)$ is much larger than the performance variation as a function of the azimuthal angle $(\phi)$. In fact, for long-term observations, averaging the LAT response over azimuth is a very good approximation. Therefore, although the standard IRFs used are parametrized in terms of $\theta$ and $\phi$, we ignore the $\phi$ dependence, i.e., $A_{\text {eff }}(E, \theta, \vec{s})$ and $D\left(E^{\prime} ; E, \theta, \vec{s}\right)$.

(2) We calculate the "observing profile" $t_{\text {obs }}(\theta ; \hat{p})$, i.e., the distribution of observing time with incident angle, by integrating the time that a particular direction in the sky is at a particular direction in the LAT reference frame. ${ }^{10}$ We can also precompute the exposure as a function of $\theta$ for each direction in the sky, $\mathcal{E}(E, \theta, \vec{s})=A_{\text {eff }}(E, \theta, \vec{s}) t_{\text {obs }}(\theta ; \hat{p})$.

(3) We assume that we can neglect the effect of the PSF. This is equivalent to assuming the PSF is small compared to changes in the product of the exposure and the source intensity. Since we are masking bright point sources, this is a reasonable approximation.

We can then express the predicted counts spectrum in terms of the livetime cube:

$$
\begin{aligned}
M\left(E^{\prime}, \hat{p}, \theta ; \vec{s}\right)= & \int S(E, \hat{p}) A_{\mathrm{eff}}(E, \theta, \vec{s}) t_{\mathrm{obs}}(\theta ; \hat{p}) \\
& \times D\left(E^{\prime} ; E, \theta, \vec{s}\right) d E .
\end{aligned}
$$

Furthermore, we do not have particularly strong a priori knowledge about the morphological details of the DM line signal, and the astrophysical backgrounds have substantial uncertainties. Therefore, for each ROI we analyze, we choose to integrate over the ROI and perform the fit only in the energy domain. We note that while some authors have chosen to retain the spatial information in their fitting procedures (e.g., Ref. [22]), others have not (e.g., Ref. [20]). While including spatial information in the fit increases sensitivity, one must chose a specific DM hypothesis to test. By integrating over the ROI, we are able to test for the existence of a monochromatic source generally.

After integrating the model over the ROI and the FOV, we obtain a predicted counts spectra that we can compare with observations:

\footnotetext{
${ }^{10}$ The gtltcube tool calculates the observing profile for each direction in the sky, which is often called the "livetime cube."
} 


$$
C\left(E^{\prime} ; \vec{s}\right)=\int^{\mathrm{ROI}} \int^{\mathrm{FOV}} M\left(E^{\prime}, \hat{p}, \theta ; \vec{s}\right) d \Omega_{\hat{v}} d \Omega .
$$

By further integrating over the fit energy band, we can obtain the total number counts predicted by the model:

$$
n^{\text {pred }}=\int C\left(E^{\prime} ; \vec{s}\right) d E^{\prime}
$$

This also allows us to split the predicted counts spectrum into a normalized probability density function $F\left(E^{\prime} ; \vec{s}\right)$ times the number of counts ( $n$, which we will treat as a free parameter of the fit):

$$
C\left(E^{\prime} ; \vec{s}\right)=n F\left(E^{\prime} ; \vec{s}\right),
$$

where $F\left(E^{\prime} ; \vec{s}\right)$ is defined by

$$
F\left(E^{\prime} ; \vec{s}\right)=\frac{C\left(E^{\prime} ; \vec{s}\right)}{n^{\text {pred }}} .
$$

Note that while $n$ is just a scalar quantity that is varied by the likelihood minimizer, $n^{\text {pred }}$ is a normalization integral that must be calculated from Eq. (C4).

In the particular case of a line search, we separate the source model into the contributions from a $\gamma$-ray line, $S_{\text {sig }}$, and those from all other astrophysical sources, $S_{\mathrm{bkg}}$, such that

$$
S(E, \hat{p})=S_{\mathrm{sig}}(E, \hat{p})+S_{\mathrm{bkg}}(E, \hat{p}) .
$$

And likewise, we separate the predicted counts distributions:

$$
C\left(E^{\prime} ; \vec{s}\right)=C_{\mathrm{sig}}\left(E^{\prime} ; \vec{s}\right)+C_{\mathrm{bkg}}\left(E^{\prime} ; \vec{s}\right) .
$$

For a binned likelihood analysis, we compute the log likelihood as the sum of the logarithm of the Poisson probability to observe $n^{\text {obs }}$ events in a particular bin in $E^{\prime}$ and $P_{\mathrm{E}}$, given that the model predicts $n^{\text {pred. }}$

$$
\ln \mathcal{L}_{b}=\sum_{i}^{\text {bins }} n_{i}^{\text {obs }} \ln n_{i}^{\text {pred }}-\sum_{i}^{\text {bins }} n_{i}^{\text {pred }}
$$

For an unbinned likelihood analysis, we instead compute the sum of the log likelihood of the individual events based on the predicted distribution:

$$
\ln \mathcal{L}_{u}=\sum_{i}^{\text {events }} \ln C\left(E_{i}^{\prime} ; \vec{s} \mid \vec{\alpha}\right)-C_{\mathrm{tot}}
$$

where $C_{\text {tot }}$ is the total number of $\gamma$ rays predicted by the model, and $\vec{\alpha}$ represents the model parameters, such as $E_{\gamma}$ and $\Gamma_{\mathrm{bkg}}$.

\section{Line search signal model}

We can factor the signal model into the photon spectrum and spatial intensity $I_{\text {sig }}(\hat{p})$, and explicitly write the photon spectrum as a delta function at the line energy $E_{\gamma}$ :

$$
S_{\text {sig }}\left(E, \hat{p} \mid E_{\gamma}\right)=I_{\text {sig }}(\hat{p}) \delta\left(E-E_{\gamma}\right) .
$$

We then express the model in terms of the total number of signal counts, $n_{\text {sig }}$, which will become a free parameter in our fit, and the total predicted number of counts, $n_{\text {sig }}^{\text {pred }}$ :

$$
\begin{aligned}
& C_{\text {sig }}\left(E^{\prime} ; \vec{s} \mid E_{\gamma}\right) \\
& =n_{\mathrm{sig}} \int^{\mathrm{ROI}} \int^{\mathrm{FOV} D\left(E^{\prime} ; E_{\gamma}, \theta, \vec{s}\right) I_{\mathrm{sig}}(\hat{p}) \mathcal{E}\left(E_{\gamma}, \hat{p}, \theta ; \vec{s}\right)} \frac{n_{\mathrm{sig}}^{\text {pred }}}{n_{\hat{v}}} d \Omega \text {, }
\end{aligned}
$$

where the normalization term $n_{\text {sig }}^{\text {pred }}$ must be calculated using

$$
\begin{aligned}
n_{\mathrm{sig}}^{\mathrm{pred}}= & \iint^{\mathrm{ROI}} \int^{\mathrm{FOV}} D\left(E^{\prime} ; E_{\gamma}, \theta, \vec{s}\right) I_{\mathrm{sig}}(\hat{p}) \\
& \times \mathcal{E}\left(E_{\gamma}, \hat{p}, \theta ; \vec{s}\right) d \Omega_{\hat{v}} d \Omega d E^{\prime} .
\end{aligned}
$$

\section{Line search background model}

Empirically, at $\mathrm{GeV}$ energies, the spectrum of diffuse emission for relatively large regions of the sky is quite smooth. Thus, in our ROIs, it can be well modeled as a power law for the relatively narrow $(\sim 1 / 2$ decade) energy intervals we are fitting. Furthermore, by design the energy dispersion is much smaller than the fit energy ranges (recall, we fit in $\pm 6 \sigma_{E}$ ranges). Thus, for the background model, we approximate $S_{\text {bkg }}(E, \hat{p})$ to have a single-power-law dependence and write the spatial dependence as $I_{\mathrm{bkg}}$. Also, the energy resolution varies fairly slowly with energy and changes only slightly across any given fit range; therefore, we treat the energy dispersion as $\delta\left(E^{\prime}-E\right)$.

With these approximations, we can express the background model in terms of the total number of counts, $n_{\mathrm{bkg}}$, which will become a free parameter in our fit:

$$
\begin{aligned}
C_{\mathrm{bkg}}\left(E^{\prime} ; \vec{s} \mid \Gamma_{\mathrm{bkg}}\right)= & n_{\mathrm{bkg}} \frac{1}{n_{\mathrm{bkg}}^{\text {pred }}}\left(\frac{E^{\prime}}{E_{0}}\right)^{-\Gamma_{\mathrm{bkg}}} \\
& \times \int^{\mathrm{ROI}} \int^{\mathrm{FOV}} I_{\mathrm{bkg}}(\hat{p}) \\
& \times \mathcal{E}\left(E^{\prime}, \hat{p}, \theta ; \vec{s}\right) d \Omega_{\hat{v}} d \Omega .
\end{aligned}
$$

Note that normalization $n_{\mathrm{bkg}}^{\text {pred }}$ depends on $\Gamma_{\mathrm{bkg}}$ and must be calculated using

$$
\begin{aligned}
n_{\mathrm{bkg}}^{\mathrm{pred}}= & \iint^{\mathrm{ROI}} \int^{\mathrm{FOV}}\left(\frac{E^{\prime}}{E_{0}}\right)^{-\Gamma_{\mathrm{bkg}}} I_{\mathrm{bkg}}(\hat{p}) \\
& \times \mathcal{E}\left(E^{\prime}, \hat{p}, \theta ; \vec{s}\right) d \Omega_{\hat{v}} d \Omega d E^{\prime} .
\end{aligned}
$$

\section{Energy dispersion parametrization}

In this paper we use two parametrizations of the energy dispersion, depending on the study being performed: 
(1) We use an energy quality estimator, $P_{\mathrm{E}}$, in our predicted counts model and integrating over $\theta$; in this case our predicted "2D" counts model depends on $E^{\prime}$ and $P_{\mathrm{E}}: C\left(E^{\prime}, P_{\mathrm{E}} ; \vec{s}\right)$. We use this parametrization for all of the fits except those listed below.

(2) We obtain a simpler "1D" model of the energy dispersion at the price of a $15 \%$ loss of sensitivity by averaging the energy dispersion over $\theta$. We use this approach when finely scanning the Galactic plane for spectral features (Sec. D 7 b) and for the pseudoexperiments we used to estimate the effective trials factor (Sec. VB) and the effects of the dips in efficiency we observed in the Limb data (Sec. VIII C 1), as it is computationally much faster than the 2D parametrization.

We derive the energy dispersion models for both formulations below.

\section{a. Energy dispersion parametrized by energy only}

We can obtain the simpler "1D" form of the energy dispersion model by averaging the instrument response across the FOV as well as the ROI.

For the signal model, the spatial integrals give the factor needed to reweight the contributions to the energy dispersion model. However, since the intensity of the signal is brightest toward the Galactic center, and in fact markedly so for some of the DM models considered, we simply pick the Galactic center out of the integral [i.e., $I_{\mathrm{sig}}(\hat{p})=$ $\left.I_{0} \delta\left(\hat{p}=\hat{p}_{\mathrm{GC}}\right)\right]$. Therefore, we can define an effective energy dispersion model:

$$
\begin{aligned}
D_{\text {eff }}\left(E^{\prime} ; E_{\gamma}, \vec{s}\right)= & \int^{\mathrm{FOV}} D\left(E^{\prime} ; E_{\gamma}, \theta, \vec{s}\right) \\
& \times \frac{I_{0} \mathcal{E}\left(E_{\gamma}, \theta ; \hat{p}_{\mathrm{GC}}, \vec{s}\right)}{n_{\mathrm{sig}}^{\text {pred }}} d \Omega_{\hat{v}} .
\end{aligned}
$$

In practice, we perform the integration by reweighting an isotropically generated MC sample of $\gamma$ rays to match the $\theta$ distribution and fitting for the energy dispersion parameters (see Appendix C, Sec. C 5).

Given the effective energy dispersion, we can write the signal model as

$$
C_{\text {sig }}\left(E^{\prime} ; \vec{s} \mid E_{\gamma}\right)=n_{\text {sig }} D_{\text {eff }}\left(E^{\prime} ; E_{\gamma}, \vec{s}\right) .
$$

For the background model, the spatial integrals give us the energy-dependent exposure correction

$$
\eta\left(E^{\prime} ; \vec{s}\right)=\int^{\mathrm{FOV}} \int^{\mathrm{ROI} I_{\mathrm{bkg}}(\hat{p}) \mathcal{E}\left(E^{\prime}, \hat{p}, \theta ; \vec{s}\right)} \frac{n_{\mathrm{bkg}}^{\mathrm{pred}}}{} d \Omega d \Omega_{\hat{v}},
$$

so that we can write the background model as

$$
C_{\mathrm{bkg}}\left(E^{\prime} ; \vec{s} \mid \Gamma_{\mathrm{bkg}}\right)=n_{\mathrm{bkg}}\left(\frac{E^{\prime}}{E_{0}}\right)^{-\Gamma_{\mathrm{bkg}}} \eta\left(E^{\prime} ; \vec{s}\right) .
$$

Putting together the signal and background models, we have

$$
C\left(E^{\prime} ; \vec{s} \mid \vec{\alpha}\right)=n_{\text {sig }} D_{\text {eff }}\left(E^{\prime} ; E_{\gamma}, \vec{s}\right)+n_{\mathrm{bkg}}\left(\frac{E^{\prime}}{E_{0}}\right)^{-\Gamma_{\mathrm{bkg}}} \eta\left(E^{\prime}, \vec{s}\right),
$$

where the model parameters are $E_{\gamma}$ (held fixed), $\Gamma_{\mathrm{bkg}}, n_{\mathrm{sig}}$ and $n_{\mathrm{bkg}}$. While $E_{\gamma}$ and $\Gamma_{\mathrm{bkg}}$ are physical quantities, we must use the exposure and intensity maps to extract the source fluxes from $n_{\text {sig }}$ and $n_{\mathrm{bkg}}$.

\section{b. Energy dispersion parametrized by energy and $P_{\mathrm{E}}$}

If we are considering an energy dispersion model that includes the energy quality estimator $P_{\mathrm{E}}$, then we must factor out $P_{\mathrm{E}}$ from the instrument response. In particular, we consider the distribution of $P_{\mathrm{E}}, w\left(P_{\mathrm{E}} ; E, \theta, \vec{s}\right)$, such that

$$
A_{\text {eff }}\left(E, \theta, P_{\mathrm{E}}, \vec{s}\right)=A_{\text {eff }}(E, \theta, \vec{s}) w\left(P_{\mathrm{E}} ; E, \theta, \vec{s}\right),
$$

with the normalization constraint

$$
\int w\left(P_{\mathrm{E}} ; E, \theta, \vec{s}\right) d P_{\mathrm{E}}=1
$$

for all $E, \theta$ and $\vec{s}$.

We can now include $P_{\mathrm{E}}$ in the expression for our expected counts spectrum and integrate over the FOV, the ROI, and $E$. In this way, we can define an effective energy dispersion model for the ROI:

$$
\begin{aligned}
& D_{\text {eff }}\left(E^{\prime} ; E_{\gamma}, P_{\mathrm{E}}, \vec{s}\right) \\
& =\int^{\mathrm{FOV}} \int^{\mathrm{ROI}} D\left(E^{\prime} ; E_{\gamma}, \theta, P_{\mathrm{E}}, \vec{s}\right) \\
& \quad \times \frac{I_{\mathrm{sig}}(\hat{p}) \mathcal{E}\left(E_{\gamma}, \hat{p}, \theta ; \vec{s}\right)}{n_{\mathrm{sig}}^{\text {pred }}} w\left(P_{\mathrm{E}} ; E_{\gamma}, \theta, \vec{s}\right) d \Omega d \Omega_{\hat{v}} .
\end{aligned}
$$

At this point, we assume that the distribution of $P_{\mathrm{E}}$ for $E_{\gamma}$ and all $\theta$ is adequately modeled by the total observed distribution of $P_{\mathrm{E}}$ in the ROI; i.e., we replace $w\left(P_{\mathrm{E}} ; E_{\gamma}, \theta, \vec{s}\right)$ with $w_{\text {sig }}\left(P_{\mathrm{E}}, \vec{s}\right)$ and remove it from the spatial integrals. Rather than obtaining $D_{\text {eff }}\left(E^{\prime} ; E_{\gamma}, P_{\mathrm{E}}, \vec{s}\right)$ by explicitly performing the above integrals, we assume that it is reasonably well modeled by a distribution of isotropically generated $\gamma$ rays (i.e., we obtain the model by interpolating in energy between the parameters obtained from fitting to the "isotropic-monochromatic" samples described in Sec. II B).

With these approximations, we can write the predicted counts distribution as 
$C_{\text {sig }}\left(E^{\prime}, P_{\mathrm{E}} ; \vec{s} \mid E_{\gamma}\right)=n_{\text {sig }} D_{\text {eff }}\left(E^{\prime} ; E_{\gamma}, P_{\mathrm{E}}, \vec{s}\right) w_{\text {sig }}\left(P_{\mathrm{E}} ; \vec{s}\right)$.

On the other hand, for the background, we are neglecting the energy dispersion and modeling the spectrum as a power law:

$$
\begin{aligned}
& C_{\mathrm{bkg}}\left(E^{\prime}, P_{\mathrm{E}} ; \vec{s} \mid \Gamma_{\mathrm{bkg}}\right) \\
& =n_{\mathrm{bkg}}\left(\frac{E^{\prime}}{E_{0}}\right)^{-\Gamma_{\mathrm{bkg}}} \int^{\mathrm{FOV}} \int^{\mathrm{ROI} I_{\mathrm{bkg}}(\hat{p}) \mathcal{E}\left(E^{\prime}, \hat{p}, \theta ; \vec{s}\right)} \\
& \quad \times w\left(P_{\mathrm{E}} ; E^{\prime}, \theta, \overrightarrow{s k g}\right) d \Omega d \Omega_{\hat{v}} .
\end{aligned}
$$

As for the signal case, we assume the distribution of $P_{\mathrm{E}}$ for all $E^{\prime}$ and $\theta$ is adequately modeled by the total observed distribution of $P_{\mathrm{E}}$ in the ROI and remove it from the spatial integrals, which we then replace with the energy-dependent exposure correction from Eq. (C18). This gives us the following background model:

$$
C_{\mathrm{bkg}}\left(E^{\prime}, P_{\mathrm{E}} ; \vec{s} \mid E_{\gamma}\right)=n_{\mathrm{bkg}}\left(\frac{E^{\prime}}{E_{0}}\right)^{-\Gamma_{\mathrm{bkg}}} \eta\left(E^{\prime} ; \vec{s}\right) w_{\mathrm{bkg}}\left(P_{\mathrm{E}} ; \vec{s}\right)
$$

Combining the signal and background models, we obtain

$$
\begin{aligned}
C\left(E^{\prime}, P_{\mathrm{E}} ; \vec{s} \mid \vec{\alpha}\right)= & n_{\mathrm{sig}} D_{\text {eff }}\left(E^{\prime} ; E_{\gamma}, P_{\mathrm{E}}, \vec{s}\right) w_{\mathrm{sig}}\left(P_{\mathrm{E}} ; \vec{s}\right) \\
& +n_{\mathrm{bkg}}\left(\frac{E^{\prime}}{E_{0}}\right)^{-\Gamma_{\mathrm{bkg}}} \eta\left(E^{\prime} ; \vec{s}\right) w_{\mathrm{bkg}}\left(P_{\mathrm{E}}, \vec{s}\right),
\end{aligned}
$$

where the fit parameters $\vec{\alpha}$ are the same as for the previous case.

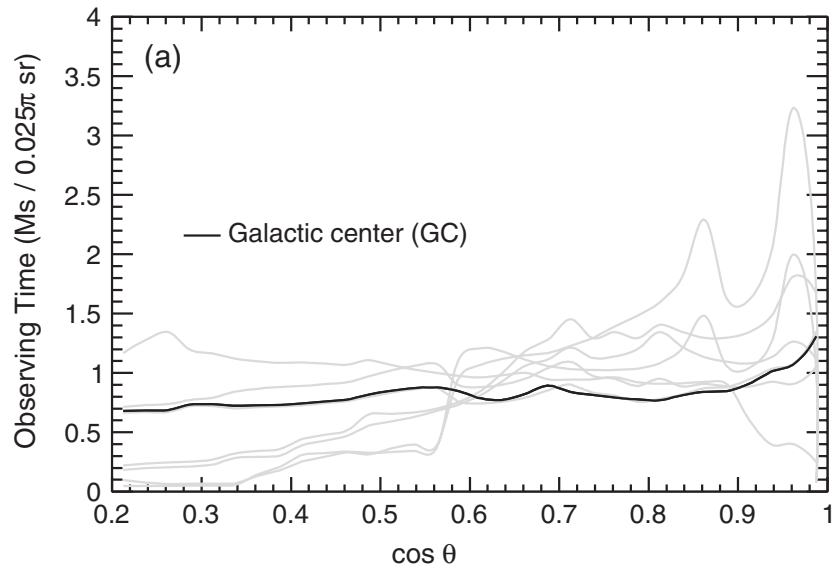

In practice, we take the model for the distribution of $P_{\mathrm{E}}$ for both the signal and background from the flight data in the ROI, i.e.,

$$
w_{\text {bkg }}\left(P_{\mathrm{E}}, \vec{s}\right)=w_{\text {sig }}\left(P_{\mathrm{E}}, \vec{s}\right)=w^{\mathrm{ROI}}\left(P_{\mathrm{E}}, \vec{s}\right) .
$$

A subtlety exists in this last approximation: the $\theta$ distribution of $\gamma$-ray directions differs for the signal and background $\gamma$ rays, because of differences in the spatial morphology, or because of CR contamination in the background $\gamma$-ray sample. This means that this last approximation might be wrong in slightly different ways when applied to the signal or background. This is the so-called "Punzi effect" [45]. We consider this further in Appendix D, Sec. D 4.

\section{Calculating the effective energy dispersion and exposure corrections}

We absorbed many details about the morphology of the flux models and spatial variations of the exposure into the calculations of effective energy dispersion and the energy-dependent exposure corrections.

Practically speaking, we can create an effective energy dispersion model with MC simulations by reweighting events from an isotropically generated sample to match a particular observing profile $t_{\mathrm{obs}}(\theta ; \hat{p})$ applying the event selection criteria and fitting the parameters of $D_{\text {eff }}\left(E^{\prime} ; E_{\gamma}, P_{\mathrm{E}}, \vec{s}\right)$ to the resulting energy dispersion distribution. The observing profiles and corresponding effective energy dispersion models for several different directions in the sky are shown in Fig. 23. Since the observing profile for the Galactic center is so close to uniform, and since the variation in resolution is already described by the $P_{\mathrm{E}}$ parameter, when generating the 2D PDF, we elected not to reweight the events and simply interpolated

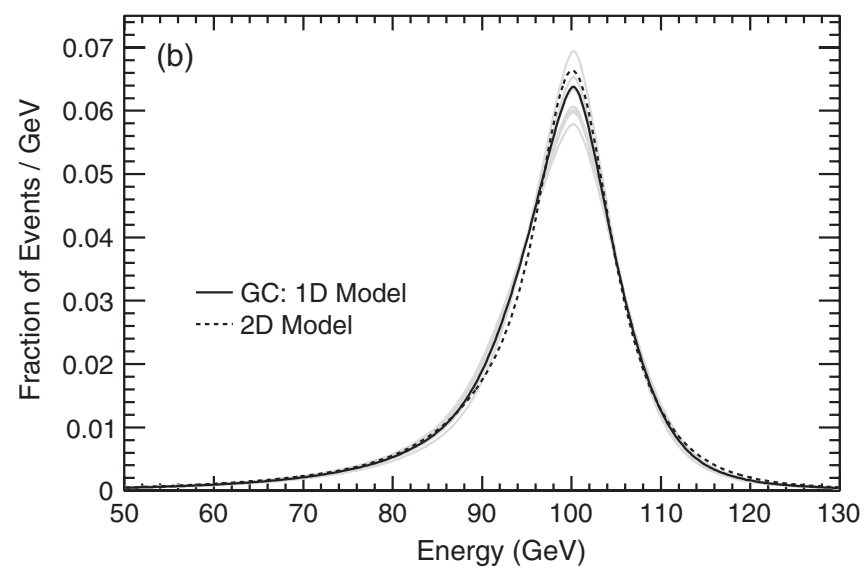

FIG. 23. Effect of variations in observing profiles on the energy dispersion model: (a) Observing profiles, $t_{\mathrm{obs}}(\theta ; \hat{p})$, for several directions with the same right ascension as the Galactic center, but different declinations $\left( \pm 30^{\circ}\right.$, $\pm 60^{\circ}$, and $\left.\pm 90^{\circ}\right)$. (b) The corresponding energy dispersion models for $E=100 \mathrm{GeV}$. 
the parameters obtained from the fits to the isotropicmonochromatic MC samples (Sec. II B).

\section{APPENDIX D: STUDIES OF SYSTEMATIC UNCERTAINTIES}

In this appendix, we provide details about sources of systematic uncertainty and describe the studies we performed to quantify the particular effects.

\section{Uncertainties and approximations of the exposure}

We convert our counts limits to flux limits by using the average exposure in the ROI [see Eq. (15)]. The LAT observes the sky with approximately uniform exposure, and the rms variation of the exposure in each ROI ranges from $|\delta \mathcal{E} / \mathcal{E}|<0.01$ in $\mathrm{R} 3$ up to $|\delta \mathcal{E} / \mathcal{E}|=0.10(0.13)$ in $\mathrm{R} 180$ at $5 \mathrm{GeV}(300 \mathrm{GeV})$. (The off-axis effective area decreases at higher energies, causing the exposure to be slightly less uniform.)

The Fermi-LAT Collaboration has estimated that the overall uncertainty of the effective area is $10 \%$ for energies $>10 \mathrm{GeV}$ and decreases to $8 \%$ at $\sim 5 \mathrm{GeV}$ [31]. For simplicity, in this analysis we have chosen to assign a $10 \%$ uncertainty from the overall effective area at all energies. This uncertainty causes a corresponding $|\delta \mathcal{E} / \mathcal{E}|=0.10$ uncertainty in the exposure. Adding this effect in quadrature with the variation in exposure between the ROIs yields $0.10<|\delta \mathcal{E} / \mathcal{E}|<0.16$ as the overall range of relative uncertainty.

\section{Uncertainties in the energy resolution}

The error in the measurement of the energy resolution was measured in beam tests and found to be better than $10 \%$ for energies up to $280 \mathrm{GeV}$ [31]. To test how a different energy resolution would affect our limits, we scaled the standard deviations of all the Gaussians in the 2D energy dispersion model (see Sec. IV) by a common scale factor of 1.1 or 0.9 , while also scaling the means to preserve the shape, and then fit to MC simulations containing a line. We found that the relative error on the bestfit number of signal events $\left(\delta n_{\text {sig }} / n_{\text {sig }}\right)$ was proportional to the width scale factor used in the fit. If the fit model was too narrow, it underfit the number of signal counts. However, if the fit model was too wide, then it overfit the number of signal counts. The constant of proportionality between $\delta n_{\text {sig }} / n_{\text {sig }}$ and the model scale factor was 0.7. Therefore, fitting with a model that was $10 \%$ too narrow would, on average, underfit the number of signal counts by $7 \%$. We found similar variation in the expected limits in background-only MC simulations.

\section{Intrinsic width of the $\gamma$-ray emission}

In the context of this analysis, any intrinsic width of the $\gamma$-ray emission, e.g., from the $Z$ width in $Z \gamma$ final states, would manifest very similarly as an unmodeled increase in the energy resolution. We note that even if the intrinsic width of the emission were $50 \%$ of the energy resolution, when convolved with the energy resolution, it would only increase the width of the observed spectral feature by $11 \%$. As stated in Appendix D, Sec. D 2, this would cause us to underestimate the signal by $7 \%$. Furthermore, for $m_{\chi}=m_{\mathrm{Z}}=91 \mathrm{GeV}$, the $\gamma$-ray threshold energy is $E_{\gamma}=68 \mathrm{GeV}$. Given that the $Z$ width is $2.5 \mathrm{GeV}$, and the energy resolution is $\Delta E \sim 5 \mathrm{GeV}$ at $68 \mathrm{GeV}$, we estimate that at worst $\delta n_{\text {sig }} / n_{\text {sig }}=0.07$ for $Z \gamma$ final states at $E_{\gamma} \simeq 68 \mathrm{GeV}$.

\section{Approximations in the energy dispersion modeling}

The $P_{\mathrm{E}}$ distribution of the events in a specific ROI and energy interval $\left[w^{\mathrm{ROI}}\left(P_{\mathrm{E}}\right)\right]$ influences the energy dispersion model $D_{\text {eff }}\left(E^{\prime} ; E_{\gamma}, P_{\mathrm{E}}\right)$ used in each fit. The same $w^{\mathrm{ROI}}\left(P_{\mathrm{E}}\right)$ was used for both the signal and background pieces of the total counts model; see Eq. (9). However, if the true $P_{\mathrm{E}}$ distribution of the signal events is slightly different, e.g., because of differences in the observing profile, or because of CR contamination in the background sample, then the approximation $w_{\text {sig }}\left(P_{\mathrm{E}}\right)=w^{\mathrm{ROI}}\left(P_{\mathrm{E}}\right)$ would be incorrect and wrongly neglect the "Punzi effect" [45]. We created $1000 \mathrm{MC}$ simulations with a signal where the "true" $w_{\text {sig }}\left(P_{\mathrm{E}}\right)$ were taken from the $50 \mathrm{GeV}$ "isotropic-monochromatic" MC data set, but the fit assumed the $w^{\mathrm{ROI}}\left(P_{\mathrm{E}}\right)$ from the P7CLEAN data with $E^{\prime} \approx$ $50 \mathrm{GeV}$. The difference is very similar to the discrepancy shown in Fig. 5 between the P7CLEAN data and the all-sky MC. We elected to use the P7CLEAN data set for this study, as the discrepancy is larger in that data set than in P7REP_CLEAN, and thus provides a more conservative estimate of the magnitude of this effect. Also, the difference between the "true" $w_{\text {sig }}\left(P_{\mathrm{E}}\right)$ and the fit $w^{\mathrm{ROI}}\left(P_{\mathrm{E}}\right)$ was somewhat larger than the $P_{\mathrm{E}}$ distribution variation we see in the data. On average, using the incorrect $w^{\mathrm{ROI}}\left(P_{\mathrm{E}}\right)$ in the fit resulted in an error on the total number of signal counts of $\delta n_{\text {sig }} / n_{\text {sig }} \leq 0.01$. Therefore, the approximation $w_{\text {bkg }}\left(P_{\mathrm{E}}\right)=w_{\text {sig }}\left(P_{\mathrm{E}}\right)=w^{\mathrm{ROI}}\left(P_{\mathrm{E}}\right)$ in the fit does not result in a large systematic effect.

Though the event incidence angle $(\theta)$ and $P_{\mathrm{E}}$ are correlated, the expected 2D energy dispersion, $D\left(E^{\prime} ; E, P_{\mathrm{E}}\right)$, varies only moderately with $\theta$. In a given $P_{\mathrm{E}}$ bin, the energy resolution for events with large $\theta$ tends to be better than for on-axis events. Since the $\theta$ distribution in the monochromatic $\mathrm{MC}$ we used to derive the $2 \mathrm{D}$ energy dispersion model is very similar to the $\theta$ distribution in the flight data, we do not expect differences in the $\theta$ distribution compared to the $\mathrm{MC}$ to introduce a large systematic effect. We reweighted the monochromatic $\mathrm{MC}$ in each $P_{\mathrm{E}}$ bin to match the $\theta$ distribution in the flight data and rederived the energy dispersion model. On average, the scale factor to convert the average widths of the nominal model to the widths of the reweighted model 
is 0.97 . Using the scaling relation derived in the previous section, the resulting relative uncertainty on $n_{\text {sig }}$ is $2 \%$ on average.

\section{Cosmic ray background contamination}

Our energy reconstruction algorithm is based on the assumption that the incoming particle is a $\gamma$ ray, and therefore that the energy deposited in the calorimeter is well described as an electromagnetic shower. For hadronic $\mathrm{CRs}$, the energy reconstruction is therefore incorrect and can create spectral artifacts. Furthermore, although care was taken to ensure that the selection criteria vary smoothly with energy for $\gamma$ rays, this was not the case for hadronic CRs. This second point implies that any spectral features caused by CRs are likely to be different for different event classes.

The P7REP_CLEAN event selection rejects CR backgrounds at a level of $10^{5}$ or higher. This makes it difficult to use MC to study the spectra of background contamination for two reasons: (i) the need to generate prohibitively large samples to have reasonable statistics for the CR backgrounds leaking through the $\gamma$-ray event selection, and (ii) by definition, the background events that survive $\gamma$-ray event selection are very unusual events, so that small problems with the fidelity of the MC simulation can easily contribute at a large enough level to invalidate predictions.

To investigate the possible effects of background contamination in our sample, we considered the set of events in the P7REP_SOURCE class that did not enter the P7REP_CLEAN class. (We note that the P7REP_CLEAN event sample is a strict subset of the P7REP_SOURCE sample.) This allows us to estimate the CR contamination in the P7REP_SOURCE class.

Figure 24 shows the fraction of events in P7REP_SOURCE that survive in the P7REP_CLEAN sample for the various
ROIs, as well as the counts spectrum of the events which do not survive into the P7REP_CLEAN sample for the R180 ROI. When we fit the counts spectrum of these events for a linelike signal using the P7REP_CLEAN to estimate the size of potential induced signals, we find that several of the fits show $>2 \sigma$ induced signals, with the induced fractional signal for this CR-rich sample reaching $f_{\mathrm{CR}}=0.05$.

To estimate the effect this CR contamination might have on the analysis performed with P7REP_CLEAN class events, we estimated the amount of background contamination in the P7REP_SOURCE sample by comparing the fraction of events in the P7REP_SOURCE sample that survive into the P7REP_CLEAN sample relative to the ratio of the acceptances, $\operatorname{Acc}\left(E^{\prime}\right)$; i.e., the effective area integrated over the field of view:

$$
\frac{n_{\mathrm{CR}}\left(E^{\prime}\right)}{n_{\gamma}\left(E^{\prime}\right)} \simeq \frac{\operatorname{Acc}_{\mathrm{CLEAN}}\left(E^{\prime}\right)}{\operatorname{Acc}_{\mathrm{SOURCE}}\left(E^{\prime}\right)} \frac{n_{\mathrm{SOURCE}}\left(E^{\prime}\right)}{n_{\mathrm{CLEAN}}\left(E^{\prime}\right)}-1
$$

The induced fractional signal from $\mathrm{CR}$ contamination in the total P7REP_SOURCE sample is smaller than in the CR-rich subsample that does not survive in the P7REP_CLEAN event class:

$$
f_{\text {SOURCE }}\left(E^{\prime}\right)=\frac{n_{\mathrm{CR}}\left(E^{\prime}\right)}{n_{\mathrm{CR}}\left(E^{\prime}\right)+n_{\gamma}\left(E^{\prime}\right)} f_{\mathrm{CR}}
$$

Combining the last two equations gives us an estimate of the induced fractional signal in the P7REP_SOURCE class:

$$
f_{\text {SOURCE }}\left(E^{\prime}\right)=f_{\mathrm{CR}}-\frac{\operatorname{Acc}_{\text {SOURCE }}\left(E^{\prime}\right)}{\operatorname{Acc}_{\text {CLEAN }}\left(E^{\prime}\right)} \frac{n_{\mathrm{CLEAN}}\left(E^{\prime}\right)}{n_{\text {SOURCE }}\left(E^{\prime}\right)} f_{\mathrm{CR}}
$$

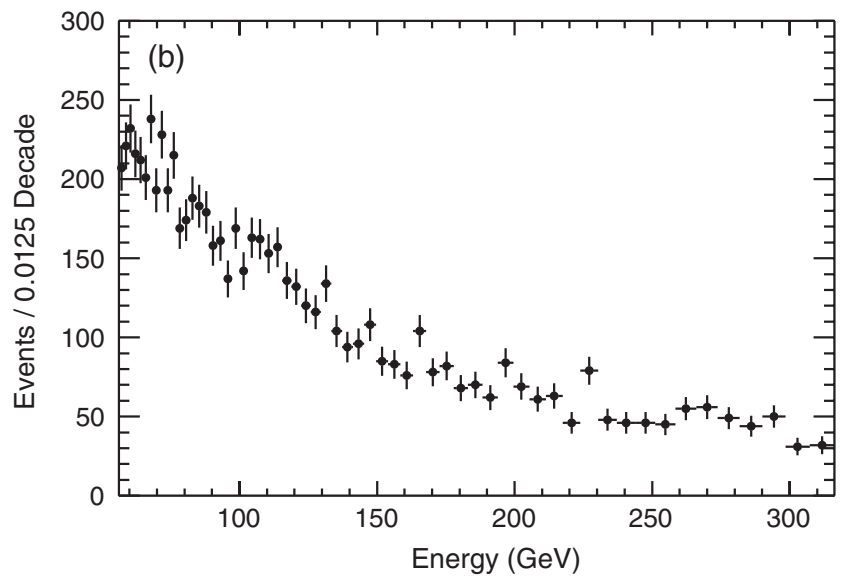

FIG. 24. CR background contamination estimation: (a) The fraction of events in the P7REP_SOURCE sample also present in the P7REP_CLEAN sample as a function of energy for all the ROIs. (b) The counts spectrum of events in the P7REP_SOURCE sample but not the P7REP_CLEAN sample for the R180 ROI. The dashed line in (a) is the ratio $\operatorname{Acc}_{\text {SOURCE }}\left(E^{\prime}\right) / \operatorname{Acc}_{\text {CLEAN }}\left(E^{\prime}\right)$. 
Based on MC simulations, we estimate that the CR contamination fraction for the P7REP_CLEAN class is less than $10 \%$ of the P7REP_SOURCE class fraction above $10 \mathrm{GeV}$. This suggests that for the P7REP_CLEAN class, CR contamination is a negligible $(\delta f<0.01)$ effect. However, residual CRs surviving from the P7REP_SOURCE to the P7REP_CLEAN data set over a narrow range of energies could induce or mask a line signal. We have not seen any evidence of such contamination, and have chosen to assign $50 \%$ of the estimated induced fractional signal for the P7REP_SOURCE event class, $f_{\text {SOURCE }}$, as the uncertainty for the P7REP_CLEAN event class. With this assignment, we found that the CR-background contamination is negligible at all energies for the R3 and R16 ROIs, but rises to $f=0.014$ for the R180 ROI at high energies.

\section{Point source contamination}

We estimated the effect of point source contamination using a similar method to the one described above for CR background contamination. First, we fit the composite spectrum of the events removed with source masking. We found fractional signals of $\delta f=0.010$ on average. Independently, we estimated that the residual contamination of the Celestial data set from point sources in our energy range is $\$ 10 \%$ (see Sec. II A). Taken together, these imply that potential induced fractional signals from point source contamination are negligible $(\delta f<0.001)$.

\section{Spectral smoothness of control samples}

\section{a. Spectral smoothness of the Earth Limb}

We used the counts spectrum of $\gamma$ rays from the Limb to estimate the size of induced fractional signals from variations in the effective area. As stated in Sec. II A, the Limb data set is obtained by selecting times when $\left|\theta_{\mathrm{r}}\right|>52^{\circ}$.

Given that the Limb photon spectrum is expected to be a featureless power law, it is an excellent control region for a spectral line search where one looks for narrow deviations from power-law behavior. We expect any linelike features observed in the Limb to be due to statistical fluctuations or variations in the effective area of the LAT over narrow ranges of energy. To estimate the size of the latter, we fit for spectral lines with our standard energy spacing and compare the measured fractional signals with the expected statistical variation given the number of events in the Limb data set.

Most of the narrow features measured in the Limb are consistent with statistical fluctuations from the powerlaw-only hypothesis. However, more than 5\% of the features have a fractional size larger than the statistical $95 \%$ containment band, suggesting that variations in the effective area are contributing as well. We approximated the size of the effective area contribution by calculating the required variation in the effective area $\left(f_{\text {Aeff }}\right)$ that allows all observed features to lie within the $95 \%$ containment band and assigning half of that variation as an estimate of the $1 \sigma$ systematic uncertainty. We found that $f_{\text {Aeff }}=$ 0.005 for low energies $(<10 \mathrm{GeV})$ and increases to $f_{\text {Aeff }}=0.015$ at $100 \mathrm{GeV}$. Above $100 \mathrm{GeV}$, the statistics from the Limb are marginal; we assign $f_{\text {Aeff }}=0.020$ and $f_{\text {Aeff }}=0.025$ as the magnitudes of the potential fractional signals at 150 and $300 \mathrm{GeV}$, respectively. We note in passing that the fit at $133 \mathrm{GeV}$ gives an anomalously large fractional signal, $f=0.14$; see Sec. VIIIC 1 for more details and discussion.

\section{b. Spectral smoothness along the Galactic plane}

Representing the complex $\gamma$-ray emission from the Galaxy as a power law is an oversimplification, and any deviations from a power law will induce signals at some level in the likelihood fit. However, it is generally assumed that any spectral features in the Galactic emission are much wider than the LAT energy resolution, and therefore that the magnitude of the induced signal is negligible.

To test this hypothesis with data, we systematically scanned across the Galactic plane and inner Galaxy, $|b|<$ $8^{\circ},|l|<90^{\circ}$. We used ROIs of $2^{\circ} \times 2^{\circ}, 4^{\circ} \times 4^{\circ}$, and $8^{\circ} \times 8^{\circ}$, and fit for a line in each energy interval. We compared these results to a second scan performed with the measured energies randomly redistributed amongst the events to remove any correlation between energy and direction.

In this study only, we allowed for both positive and negative deviations from a power law (i.e., we allowed $n_{\text {sig }}$ to be negative) to estimate the extent to which a true signal might be masked by non-power-law behavior of the background. Accordingly, we define the signed significance as $s_{\text {local }}= \pm \sqrt{\mathrm{TS}}$, where the sign matches the sign of the deviation. For this study, we adopted an upper limit of $56 \mathrm{GeV}(100 \mathrm{GeV})$ for the energy range in the $2^{\circ} \times 2^{\circ}\left(8^{\circ} \times 8^{\circ}\right)$ ROIs to avoid having the minimizer step into a parameter range where the likelihood function is negative. Given the large number of fits performed, we used the simpler " $1 \mathrm{D}$ " energy dispersion model (see Sec. IV), which does not include $P_{\mathrm{E}}$, and performed binned likelihood fits. Finally, we scanned in $b$ and $l$ using step sizes of $\frac{1}{2}$ the ROI width; thus, each ROI overlaps by $50 \%$ with the four nearest neighbors. However, all the results shown here were made using only a set of nonoverlapping ROIs obtained by removing every other step from the scan.

Figure 25 shows the distribution of signed significances for the scan along the Galactic plane using $2^{\circ} \times 2^{\circ}$ ROIs. For comparison, we have overlaid the distribution for the energy-shuffled data. We see that the flight data match the shuffled data very well; this was also true of the scan using $4^{\circ} \times 4^{\circ}$ and $8^{\circ} \times 8^{\circ}$ ROIs. Furthermore, in each case the distributions were consistent with Gaussians with unit width and zero mean, suggesting 

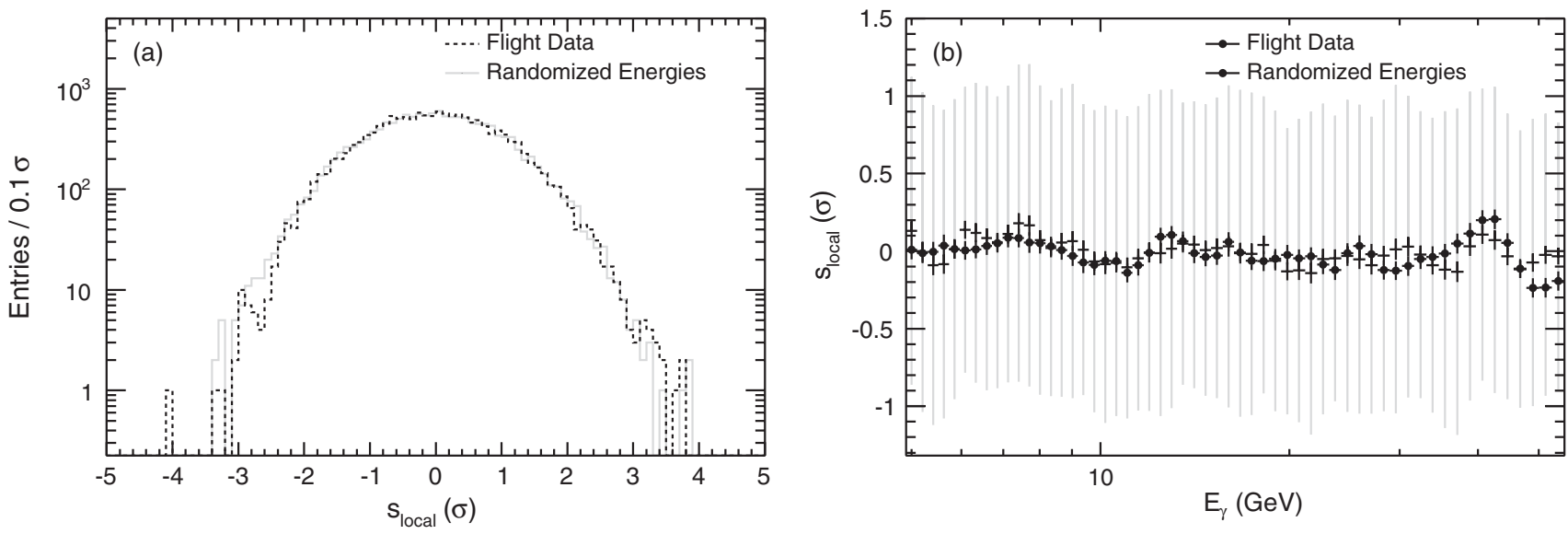

FIG. 25. Distribution of signed significances for ROIs along the Galactic plane for all $E_{\gamma}<56 \mathrm{GeV}$ : (a) for $2^{\circ} \times 2^{\circ} \mathrm{ROIs}$, and (b) mean $s_{\text {local }}$ versus $E_{\gamma}$; the large gray error bars show the rms of the distribution at a given energy, and the small black error bars show the error on the mean. Note that these results were made using only a set of nonoverlapping ROIs.

that describing the background as a power law is a good approximation.

On the other hand, the distribution of $s_{\text {local }}$ at any given energy for ROIs of a particular size tended to be slightly narrower than for the full distribution, and the means were inconsistent with zero at many energies.

For $2^{\circ} \times 2^{\circ}$ ROIs, the means of the $s_{\text {local }}$ distributions were $\left|\overline{s_{\text {local }}}\right|<0.2 \sigma$ for $E_{\gamma}<56 \mathrm{GeV}$, while for the $8^{\circ} \times$ $8^{\circ}$ ROIs they were $\left|\overline{s_{\text {local }}}\right|<0.8 \sigma$ for $E_{\gamma}<100 \mathrm{GeV}$. The corresponding means of the fractional signal distribution were small, but inconsistent with zero at many energies: rising from $|\bar{f}|=0.008$ below $10 \mathrm{GeV}$, to $|\bar{f}|=0.018$ at $30 \mathrm{GeV}$, and to $|\bar{f}|<0.042$ at $E_{\gamma}=56 \mathrm{GeV}$. Interestingly, this effect is present both in the flight data and in the sample of events with shuffled energies, suggesting that it may be related to the overall distribution of counts with energy, rather than to correlations between energies and directions. We also note that the overlapping energy ranges introduce correlations in the means of the $s_{\text {local }}$ distributions at different fit energies. However, we have chosen to assign $25 \%$ of the magnitude of the deviations of $|\bar{f}|$ from zero as a potential systematic uncertainty for the larger ROIs (R41, R90, R180), rising to $\delta f=0.02$ at $300 \mathrm{GeV}$. This is an empirically motivated choice. We believe that this study gives a reasonable measure of the

TABLE VI. Fractional signal $f$ from fits to 1000 broken power-law MC simulations with various break sizes.

\begin{tabular}{lcc}
\hline \hline$\Gamma_{1}$ & $\Gamma_{2}$ & $f$ \\
\hline 2.50 & 2.55 & 0.019 \\
2.50 & 2.60 & 0.021 \\
2.50 & 2.70 & 0.062 \\
2.00 & 3.00 & 0.085 \\
2.00 & 4.00 & 0.147 \\
2.00 & 6.00 & 0.233 \\
\hline \hline
\end{tabular}

non-power-law-like behavior for relatively large regions of the sky.

\section{c. Induced signals from limitations in the background modeling}

Here we quantify the fractional signal expected if the true spectrum is a broken power law, with the spectral index changing from $\Gamma_{1}$ and $\Gamma_{2}$ at the line-fit energy. We created 1000 MC simulations with broken power-law spectra. The fractional signal size for various break sizes is given in Table VI. We see that a true broken power law spectrum could mimic a linelike feature, though a relatively large break would be needed to induce a fractionally large signal.

Although we could in principle distinguish between a broken power law and a linelike signal on a power-law background given large statistics, in practice this is only possible at the lowest energies and for the largest ROI because of the relatively narrow energy ranges used in our fits. Since the smaller ROIs are more likely to be dominated by local variations in the diffuse $\gamma$-ray emission, and thus more likely to depart from the generally power-law-like behavior described in Appendix D, Sec. D $7 \mathrm{~b}$, we have chosen to assign the induced fraction signal of $\delta f=0.019$ for a broken power law with a small change in index $\left(\Gamma_{1}=2.5\right.$ to $\left.\Gamma_{2}=2.55\right)$ as the potential systematic uncertainty for our smaller ROIs (R3 and R16).

\section{APPENDIX E: 95\% CONFIDENCE LEVEL LIMITS}

We present the 95\% C.L. flux upper limits derived for each of our 5 ROIs in Tables VII, VIII, IX, and X. We also give the annihilation-cross-section upper limits for the DM profiles in each ROI where sensitivity to that DM model has been optimized (see Sec. III): R3 (contracted NFW profile), R16 (Einasto profile), R41 (NFW profile), and R90 (isothermal profile), and the decay lifetime lower limit for R180. 
TABLE VII. $95 \%$ confidence level limits from all ROIs for fit energies from $5-11.48 \mathrm{GeV}$. The first column for each ROI is the $\left(\Phi_{\gamma \gamma}\right)$ upper limit in $10^{-9} \mathrm{~cm}^{-2} \mathrm{~s}^{-1}$. The second column for each is the upper limit on $\langle\sigma v\rangle_{\gamma \gamma}$ in $10^{-28} \mathrm{~cm}^{-3} \mathrm{~s}^{-1}$ for the DM profile for which that ROI is optimal. For R180, we give the lower limit on $\tau_{\gamma \nu}(\mathrm{NFW})$ in $10^{29} \mathrm{~s}$. Note that for $\tau_{\gamma \nu}$, the energy is $m_{\chi} / 2$.

\begin{tabular}{|c|c|c|c|c|c|c|c|c|}
\hline \multirow[b]{2}{*}{ Energy $(\mathrm{GeV})$} & \multicolumn{2}{|c|}{ R16 } & \multicolumn{2}{|r|}{ R41 } & \multicolumn{2}{|c|}{ R90 } & \multicolumn{2}{|c|}{ R180 } \\
\hline & $\Phi_{\gamma \gamma}$ & $\langle\sigma v\rangle_{\gamma \gamma}$ Ein & $\Phi_{\gamma \gamma}$ & $\langle\sigma v\rangle_{\gamma \gamma} \mathrm{NFW}$ & $\Phi_{\gamma \gamma}$ & $\langle\sigma v\rangle_{\gamma \gamma}$ Iso & $\Phi_{\gamma \gamma}$ & $\tau_{\gamma \nu} \mathrm{NFW}$ \\
\hline 5.00 & 3.97 & 0.15 & 4.59 & 0.17 & 4.32 & 0.20 & 3.54 & 5.52 \\
\hline 5.20 & 2.96 & 0.12 & 2.53 & 0.10 & 3.09 & 0.15 & 3.36 & 5.59 \\
\hline 5.41 & 2.25 & 0.10 & 3.44 & 0.15 & 3.60 & 0.19 & 4.28 & 4.22 \\
\hline 5.62 & 1.83 & 0.09 & 4.58 & 0.21 & 4.24 & 0.24 & 5.16 & 3.37 \\
\hline 5.85 & 1.90 & 0.10 & 5.82 & 0.29 & 5.55 & 0.34 & 7.91 & 2.11 \\
\hline 6.08 & 2.03 & 0.11 & 6.21 & 0.34 & 7.19 & 0.48 & 11.69 & 1.37 \\
\hline 6.33 & 2.22 & 0.13 & 5.47 & 0.32 & 6.75 & 0.49 & 11.83 & 1.30 \\
\hline 6.58 & 2.21 & 0.14 & 4.11 & 0.26 & 6.28 & 0.49 & 9.21 & 1.61 \\
\hline 6.84 & 2.06 & 0.14 & 2.90 & 0.20 & 5.60 & 0.47 & 7.99 & 1.79 \\
\hline 7.12 & 2.05 & 0.15 & 2.17 & 0.16 & 5.48 & 0.50 & 7.37 & 1.86 \\
\hline 7.40 & 1.16 & 0.09 & 1.46 & 0.12 & 3.90 & 0.39 & 5.41 & 2.44 \\
\hline 7.70 & 0.56 & 0.05 & 1.49 & 0.13 & 2.16 & 0.23 & 2.67 & 4.74 \\
\hline 8.01 & 0.86 & 0.08 & 2.07 & 0.20 & 2.02 & 0.23 & 2.25 & 5.42 \\
\hline 8.33 & 1.51 & 0.16 & 1.39 & 0.14 & 1.38 & 0.17 & 2.12 & 5.54 \\
\hline 8.67 & 1.36 & 0.15 & 1.05 & 0.12 & 1.15 & 0.16 & 1.52 & 7.41 \\
\hline 9.02 & 1.08 & 0.13 & 0.91 & 0.11 & 1.41 & 0.21 & 1.18 & 9.15 \\
\hline 9.39 & 0.89 & 0.12 & 1.27 & 0.17 & 1.64 & 0.26 & 1.20 & 8.68 \\
\hline 9.77 & 0.73 & 0.10 & 1.45 & 0.20 & 1.36 & 0.24 & 1.14 & 8.78 \\
\hline 10.17 & 0.51 & 0.08 & 1.18 & 0.18 & 1.94 & 0.36 & 1.50 & 6.40 \\
\hline 10.59 & 0.87 & 0.15 & 1.51 & 0.25 & 1.60 & 0.33 & 1.54 & 5.98 \\
\hline 11.02 & 1.82 & 0.33 & 1.84 & 0.33 & 1.31 & 0.29 & 1.54 & 5.74 \\
\hline 11.48 & 1.48 & 0.29 & 1.85 & 0.36 & 1.86 & 0.44 & 1.93 & 4.42 \\
\hline
\end{tabular}

TABLE VIII. $\quad 95 \%$ confidence level limits from all ROIs for fit energies from 11.96-29.57 GeV. The first column for each ROI is the $\left(\Phi_{\gamma \gamma}\right)$ upper limit in $10^{-9} \mathrm{~cm}^{-3} \mathrm{~s}^{-1}$. The second column for each is the upper limit on $\langle\sigma v\rangle_{\gamma \gamma}$ in $10^{-28} \mathrm{~cm}^{-3} \mathrm{~s}^{-1}$ for the DM profile for which that ROI is optimal. For R180, we give the lower limit on $\tau_{\gamma \nu}(\mathrm{NFW})$ in $10^{29} \mathrm{~s}$. Note that for $\tau_{\gamma \nu}$, the energy is $m_{\chi} / 2$.

\begin{tabular}{|c|c|c|c|c|c|c|c|c|}
\hline \multirow[b]{2}{*}{ Energy $(\mathrm{GeV})$} & \multicolumn{2}{|c|}{$\mathrm{R} 16$} & \multicolumn{2}{|r|}{$\mathrm{R} 41$} & \multicolumn{2}{|c|}{ R90 } & \multicolumn{2}{|c|}{ R180 } \\
\hline & $\Phi_{\gamma \gamma}$ & $\langle\sigma v\rangle_{\gamma \gamma}$ Ein & $\Phi_{\gamma \gamma}$ & $\langle\sigma v\rangle_{\gamma \gamma} \mathrm{NFW}$ & $\Phi_{\gamma \gamma}$ & $\langle\sigma v\rangle_{\gamma \gamma}$ Iso & $\Phi_{\gamma \gamma}$ & $\tau_{\gamma X} \mathrm{NFW}$ \\
\hline 11.96 & 0.66 & 0.14 & 1.04 & 0.22 & 1.67 & 0.43 & 2.52 & 3.24 \\
\hline 12.46 & 0.50 & 0.12 & 0.92 & 0.21 & 2.26 & 0.63 & 4.80 & 1.63 \\
\hline 12.98 & 1.26 & 0.31 & 1.14 & 0.28 & 2.47 & 0.75 & 5.19 & 1.45 \\
\hline 13.53 & 1.55 & 0.42 & 1.32 & 0.36 & 1.54 & 0.51 & 3.46 & 2.09 \\
\hline 14.10 & 0.97 & 0.29 & 0.87 & 0.25 & 0.73 & 0.26 & 1.74 & 3.99 \\
\hline 14.70 & 0.43 & 0.14 & 0.84 & 0.27 & 0.74 & 0.29 & 1.32 & 5.03 \\
\hline 15.33 & 0.40 & 0.14 & 0.95 & 0.33 & 1.31 & 0.56 & 1.61 & 3.95 \\
\hline 15.99 & 0.37 & 0.14 & 1.13 & 0.42 & 1.85 & 0.86 & 2.70 & 2.27 \\
\hline 16.69 & 0.39 & 0.16 & 1.56 & 0.64 & 1.72 & 0.87 & 2.47 & 2.37 \\
\hline 17.42 & 0.61 & 0.27 & 1.93 & 0.86 & 2.46 & 1.35 & 2.93 & 1.92 \\
\hline 18.18 & 0.70 & 0.34 & 1.78 & 0.87 & 2.15 & 1.28 & 2.45 & 2.20 \\
\hline 18.99 & 0.55 & 0.30 & 1.55 & 0.83 & 1.78 & 1.16 & 1.91 & 2.70 \\
\hline 19.84 & 0.46 & 0.27 & 1.50 & 0.87 & 1.90 & 1.35 & 2.04 & 2.41 \\
\hline 20.73 & 0.45 & 0.29 & 0.71 & 0.45 & 0.80 & 0.62 & 0.88 & 5.35 \\
\hline 21.66 & 0.42 & 0.29 & 0.53 & 0.36 & 0.55 & 0.47 & 0.87 & 5.18 \\
\hline 22.64 & 0.50 & 0.38 & 0.36 & 0.28 & 0.40 & 0.37 & 0.75 & 5.76 \\
\hline 23.66 & 0.90 & 0.74 & 0.71 & 0.59 & 0.69 & 0.70 & 1.00 & 4.12 \\
\hline 24.74 & 0.77 & 0.70 & 0.82 & 0.74 & 1.61 & 1.78 & 1.80 & 2.19 \\
\hline 25.86 & 0.81 & 0.80 & 1.50 & 1.48 & 1.96 & 2.37 & 2.25 & 1.68 \\
\hline 27.04 & 0.72 & 0.78 & 1.21 & 1.30 & 1.37 & 1.81 & 1.14 & 3.18 \\
\hline 28.28 & 0.37 & 0.43 & 0.92 & 1.09 & 1.68 & 2.43 & 1.34 & 2.58 \\
\hline 29.57 & 0.24 & 0.32 & 0.62 & 0.79 & 1.21 & 1.91 & 1.43 & 2.31 \\
\hline
\end{tabular}


TABLE IX. $\quad 95 \%$ confidence level limits from all ROIs for fit energies from 30.93-83.43 GeV. The first column for each ROI is the $\left(\Phi_{\gamma \gamma}\right)$ upper limit in $10^{-9} \mathrm{~cm}^{-3} \mathrm{~s}^{-1}$. The second column for each is the upper limit on $\langle\sigma v\rangle_{\gamma \gamma}$ in $10^{-28} \mathrm{~cm}^{-3} \mathrm{~s}^{-1}$ for the DM profile for which that ROI is optimal. For R180, we give lower limit on $\tau_{\gamma \nu}(\mathrm{NFW})$ in $10^{29} \mathrm{~s}$. Note that for $\tau_{\gamma \nu}$, the energy is $m_{\chi} / 2$.

\begin{tabular}{|c|c|c|c|c|c|c|c|c|c|c|}
\hline \multirow[b]{2}{*}{ Energy $(\mathrm{GeV})$} & \multirow{2}{*}{\multicolumn{2}{|c|}{$\begin{array}{l}\mathrm{R} 3 \\
\langle\sigma v\rangle_{\gamma \gamma} \mathrm{NFWc}\end{array}$}} & \multirow{2}{*}{\multicolumn{2}{|c|}{$\begin{array}{l}\mathrm{R} 16 \\
\langle\sigma v\rangle_{\gamma \gamma} \text { Ein }\end{array}$}} & \multirow{2}{*}{\multicolumn{2}{|c|}{$\begin{array}{l}\mathrm{R} 41 \\
\langle\sigma v\rangle_{\gamma \gamma} \mathrm{NFW}\end{array}$}} & \multicolumn{2}{|r|}{ R90 } & \multicolumn{2}{|c|}{ R180 } \\
\hline & & & & & & & $\Phi_{\gamma \gamma}$ & $\langle\sigma v\rangle_{\gamma \gamma}$ Iso & $\Phi_{\gamma \gamma}$ & $\tau_{\gamma X} \mathrm{NFW}$ \\
\hline 30.93 & 0.08 & 0.07 & 0.18 & 0.26 & 0.32 & 0.45 & 0.36 & 0.62 & 0.68 & 4.67 \\
\hline 32.36 & 0.07 & 0.07 & 0.19 & 0.29 & 0.24 & 0.36 & 0.29 & 0.56 & 0.59 & 5.13 \\
\hline 33.85 & 0.07 & 0.08 & 0.22 & 0.37 & 0.21 & 0.35 & 0.30 & 0.62 & 0.36 & 7.97 \\
\hline 35.42 & 0.12 & 0.13 & 0.58 & 1.07 & 0.72 & 1.33 & 1.10 & 2.51 & 0.95 & 2.90 \\
\hline 37.07 & 0.29 & 0.36 & 0.66 & 1.34 & 1.04 & 2.10 & 1.38 & 3.44 & 0.97 & 2.73 \\
\hline 38.80 & 0.23 & 0.31 & 0.45 & 1.00 & 1.07 & 2.38 & 0.80 & 2.17 & 0.89 & 2.82 \\
\hline 40.62 & 0.25 & 0.38 & 0.36 & 0.89 & 1.07 & 2.60 & 0.77 & 2.29 & 1.27 & 1.89 \\
\hline 42.54 & 0.46 & 0.75 & 0.65 & 1.74 & 1.40 & 3.73 & 1.35 & 4.43 & 1.98 & 1.16 \\
\hline 44.55 & 0.43 & 0.78 & 0.46 & 1.35 & 0.87 & 2.53 & 0.70 & 2.52 & 0.66 & 3.32 \\
\hline 46.66 & 0.34 & 0.67 & 0.50 & 1.62 & 0.66 & 2.13 & 0.57 & 2.27 & 0.56 & 3.73 \\
\hline 48.88 & 0.27 & 0.58 & 0.35 & 1.24 & 0.29 & 1.00 & 0.43 & 1.86 & 0.59 & 3.37 \\
\hline 51.22 & 0.14 & 0.33 & 0.16 & 0.63 & 0.24 & 0.94 & 0.38 & 1.82 & 0.73 & 2.62 \\
\hline 53.69 & 0.15 & 0.39 & 0.21 & 0.90 & 0.24 & 1.02 & 0.37 & 1.91 & 0.73 & 2.49 \\
\hline 56.30 & 0.14 & 0.41 & 0.50 & 2.35 & 0.69 & 3.23 & 0.99 & 5.68 & 1.32 & 1.32 \\
\hline 59.05 & 0.11 & 0.34 & 0.32 & 1.63 & 0.46 & 2.39 & 0.60 & 3.78 & 0.50 & 3.32 \\
\hline 61.96 & 0.09 & 0.33 & 0.35 & 1.98 & 0.34 & 1.94 & 0.66 & 4.57 & 0.62 & 2.56 \\
\hline 65.04 & 0.14 & 0.55 & 0.22 & 1.38 & 0.36 & 2.26 & 0.38 & 2.89 & 0.66 & 2.28 \\
\hline 68.29 & 0.22 & 0.91 & 0.37 & 2.54 & 0.47 & 3.23 & 0.41 & 3.50 & 0.87 & 1.64 \\
\hline 71.75 & 0.18 & 0.82 & 0.44 & 3.32 & 0.52 & 3.95 & 0.73 & 6.78 & 1.12 & 1.21 \\
\hline 75.41 & 0.13 & 0.64 & 0.29 & 2.47 & 0.25 & 2.08 & 0.57 & 5.88 & 0.70 & 1.85 \\
\hline 79.30 & 0.07 & 0.39 & 0.10 & 0.93 & 0.20 & 1.85 & 0.28 & 3.21 & 0.32 & 3.91 \\
\hline 83.43 & 0.06 & 0.39 & 0.09 & 0.97 & 0.22 & 2.30 & 0.29 & 3.67 & 0.42 & 2.77 \\
\hline
\end{tabular}

TABLE X. $95 \%$ confidence level limits from all ROIs for fit energies from $87.82-300 \mathrm{GeV}$. The first column for each ROI is the $\left(\Phi_{\gamma \gamma}\right)$ upper limit in $10^{-9} \mathrm{~cm}^{-3} \mathrm{~s}^{-1}$. The second column for each is the upper limit on $\langle\sigma v\rangle_{\gamma \gamma}$ in $10^{-28} \mathrm{~cm}^{-3} \mathrm{~s}^{-1}$ for the DM profile for which that ROI is optimal. For R180, we give the lower limit on $\tau_{\gamma \nu}(\mathrm{NFW})$ in $10^{29} \mathrm{~s}$. Note that for $\tau_{\gamma \nu}$, the energy is $m_{\chi} / 2$.

\begin{tabular}{|c|c|c|c|c|c|c|c|c|c|c|}
\hline \multirow[b]{2}{*}{ Energy $(\mathrm{GeV})$} & \multirow[b]{2}{*}{$\Phi_{\gamma \gamma}$} & \multirow{2}{*}{$\begin{array}{l}\mathrm{R} 3 \\
\langle\sigma v\rangle_{\gamma \gamma} \mathrm{NFWc}\end{array}$} & \multirow{2}{*}{\multicolumn{2}{|c|}{$\begin{array}{l}\mathrm{R} 16 \\
\langle\sigma v\rangle_{\gamma \gamma} \text { Ein }\end{array}$}} & \multirow{2}{*}{\multicolumn{2}{|c|}{$\begin{array}{l}\mathrm{R} 41 \\
\langle\sigma v\rangle_{\gamma \gamma} \mathrm{NFW}\end{array}$}} & \multicolumn{2}{|r|}{ R90 } & \multicolumn{2}{|c|}{$\mathrm{R} 180$} \\
\hline & & & & & & & $\Phi_{\gamma \gamma}$ & $\langle\sigma v\rangle_{\gamma \gamma}$ Iso & $\Phi_{\gamma \gamma}$ & $\tau_{\gamma X} \mathrm{NFW}$ \\
\hline 87.82 & 0.08 & 0.56 & 0.18 & 2.02 & 0.27 & 3.07 & 0.25 & 3.53 & 0.71 & 1.57 \\
\hline 92.51 & 0.04 & 0.34 & 0.10 & 1.29 & 0.34 & 4.28 & 0.40 & 6.25 & 0.67 & 1.59 \\
\hline 97.50 & 0.06 & 0.48 & 0.08 & 1.18 & 0.33 & 4.66 & 0.55 & 9.44 & 0.54 & 1.86 \\
\hline 102.82 & 0.11 & 1.05 & 0.14 & 2.22 & 0.48 & 7.47 & 0.57 & 11.00 & 0.46 & 2.09 \\
\hline 108.49 & 0.06 & 0.67 & 0.22 & 3.81 & 0.45 & 7.85 & 0.32 & 6.73 & 0.45 & 2.01 \\
\hline 114.51 & 0.10 & 1.13 & 0.33 & 6.49 & 0.37 & 7.19 & 0.19 & 4.52 & 0.28 & 3.03 \\
\hline 120.89 & 0.15 & 2.01 & 0.42 & 9.01 & 0.30 & 6.50 & 0.23 & 6.00 & 0.46 & 1.77 \\
\hline 127.66 & 0.28 & 4.09 & 0.37 & 8.94 & 0.42 & 10.15 & 0.51 & 15.08 & 0.50 & 1.52 \\
\hline 134.86 & 0.31 & 5.05 & 0.38 & 10.32 & 0.51 & 13.59 & 0.63 & 20.86 & 0.63 & 1.15 \\
\hline 142.51 & 0.25 & 4.52 & 0.28 & 8.28 & 0.35 & 10.46 & 0.52 & 19.23 & 0.47 & 1.46 \\
\hline 150.66 & 0.11 & 2.16 & 0.14 & 4.78 & 0.24 & 7.91 & 0.39 & 16.01 & 0.47 & 1.37 \\
\hline 159.32 & 0.06 & 1.42 & 0.18 & 6.80 & 0.16 & 5.97 & 0.28 & 12.87 & 0.28 & 2.19 \\
\hline 168.56 & 0.06 & 1.59 & 0.20 & 8.63 & 0.15 & 6.13 & 0.36 & 18.31 & 0.37 & 1.57 \\
\hline 178.41 & 0.12 & 3.46 & 0.20 & 9.62 & 0.21 & 10.01 & 0.37 & 21.20 & 0.34 & 1.60 \\
\hline 188.92 & 0.11 & 3.50 & 0.14 & 7.50 & 0.10 & 5.17 & 0.15 & 9.65 & 0.21 & 2.41 \\
\hline 200.15 & 0.08 & 2.85 & 0.12 & 7.33 & 0.10 & 5.75 & 0.09 & 6.70 & 0.17 & 2.83 \\
\hline 212.16 & 0.05 & 2.18 & 0.14 & 9.25 & 0.13 & 8.52 & 0.08 & 6.71 & 0.13 & 3.48 \\
\hline 225.08 & 0.07 & 3.41 & 0.06 & 4.15 & 0.10 & 7.55 & 0.06 & 5.95 & 0.11 & 3.98 \\
\hline 239.01 & 0.04 & 2.02 & 0.05 & 4.41 & 0.11 & 9.18 & 0.07 & 7.74 & 0.12 & 3.48 \\
\hline 254.05 & 0.05 & 2.99 & 0.08 & 7.69 & 0.16 & 15.40 & 0.16 & 18.13 & 0.14 & 2.82 \\
\hline 270.33 & 0.04 & 2.58 & 0.09 & 10.26 & 0.12 & 12.94 & 0.13 & 16.57 & 0.14 & 2.68 \\
\hline 300.00 & 0.04 & 3.29 & 0.13 & 17.62 & 0.23 & 31.02 & 0.30 & 48.83 & 0.35 & 0.93 \\
\hline
\end{tabular}


[1] P. Ade et al. (Planck Collaboration), arXiv:1303.5076.

[2] S.P. Martin, arXiv:hep-ph/9709356.

[3] D. Chung, L. Everett, G. Kane, S. King, J. Lykken, and L. Wang, Phys. Rep. 407, 1 (2005).

[4] L. Pape and D. Treille, Rep. Prog. Phys. 69, 2843 (2006).

[5] G. Jungman, M. Kamionkowski, and K. Griest, Phys. Rep. 267, 195 (1996).

[6] G. Bertone and D. Merritt, Mod. Phys. Lett. A 20, 1021 (2005).

[7] L. Bergstrom and P. Ullio, Nucl. Phys. B504, 27 (1997).

[8] S. Matsumoto, J. Sato, and Y. Sato, arXiv:hep-ph/ 0505160.

[9] F. Ferrer, L. M. Krauss, and S. Profumo, Phys. Rev. D 74, 115007 (2006).

[10] M. Gustafsson, E. Lundström, L. Bergström, and J. Edsjö, Phys. Rev. Lett. 99, 041301 (2007).

[11] S. Profumo, Phys. Rev. D 78, 023507 (2008).

[12] G. Bertone, W. Buchmuller, L. Covi, and A. Ibarra, J. Cosmol. Astropart. Phys. 11 (2007) 003.

[13] M. Gustafsson, T. Hambye, and T. Scarna, Phys. Lett. B 724, 288 (2013).

[14] A. Ibarra and D. Tran, Phys. Rev. Lett. 100, 061301 (2008).

[15] T. Bringmann, L. Bergstrom, and J. Edsjo, J. High Energy Phys. 01 (2008) 049.

[16] W.B. Atwood et al. (Fermi-LAT Collaboration), Astrophys. J. 697, 1071 (2009).

[17] A. A. Abdo et al. (Fermi-LAT Collaboration), Phys. Rev. Lett. 104, 091302 (2010).

[18] M. Ackermann et al. (Fermi-LAT Collaboration), Phys. Rev. D 86, 022002 (2012).

[19] T. Bringmann, X. Huang, A. Ibarra, S. Vogl, and C. Weniger, J. Cosmol. Astropart. Phys. 07 (2012) 054.

[20] C. Weniger, J. Cosmol. Astropart. Phys. 08 (2012) 007.

[21] E. Tempel, A. Hektor, and M. Raidal, J. Cosmol. Astropart. Phys. 09 (2012) 032.

[22] M. Su and D. P. Finkbeiner, arXiv:1206.1616.

[23] A. Hektor, M. Raidal, and E. Tempel, Astrophys. J. 762, L22 (2013).
[24] M. Su and D.P. Finkbeiner, arXiv:1207.7060.

[25] A. Hektor, M. Raidal, and E. Tempel, arXiv:1208.1996.

[26] A. Geringer-Sameth and S. M. Koushiappas, Phys. Rev. D 86, 021302 (2012).

[27] D. Whiteson, J. Cosmol. Astropart. Phys. 11 (2012) 008.

[28] A. Hektor, M. Raidal, and E. Tempel, arXiv:1209.4548.

[29] D. P. Finkbeiner, M. Su, and C. Weniger, J. Cosmol. Astropart. Phys. 01 (2013) 029.

[30] A. Boyarsky, D. Malyshev, and O. Ruchayskiy, Phys. Dark Univ. 2, 90 (2013).

[31] M. Ackermann et al. (Fermi-LAT Collaboration), Astrophys. J. Suppl. Ser. 203, 4 (2012).

[32] J. Bregeon, E. Charles, and M. Wood (Fermi-LAT Collaboration), arXiv:1304.5456.

[33] P. L. Nolan et al. (Fermi-LAT Collaboration), Astrophys. J. Suppl. Ser. 199, 31 (2012).

[34] S. Agostinelli et al. (GEANT4 Collaboration), Nucl. Instrum. Methods Phys. Res., Sect. A 506, 250 (2003).

[35] J. F. Navarro, C. S. Frenk, and S. D. M. White, Astrophys. J. 462, 563 (1996).

[36] J.F. Navarro, A. Ludlow, V. Springel, J. Wang, M. Vogelsberger, S. D. M. White, A. Jenkins, C. S. Frenk, and A. Helmi, Mon. Not. R. Astron. Soc. 402, 21 (2010).

[37] J. N. Bahcall and R. M. Soneira, Astrophys. J. Suppl. Ser. 44, 73 (1980).

[38] M. Gustafsson, M. Fairbairn, and J. Sommer-Larsen, Phys. Rev. D 74, 123522 (2006).

[39] R. Catena and P. Ullio, J. Cosomol. Astropart. Phys. 08 (2010) 004.

[40] P. Salucci, F. Nesti, G. Gentile, and C. Martins, Astron. Astrophys. 523, A83 (2010).

[41] W. Verkerke and D. Kirkby, arXiv:physics/0306116.

[42] H. Chernoff, Ann. Math. Stat. 25, 573 (1954).

[43] W.B. Atwood et al. (Fermi-LAT Collaboration), arXiv:1303.3514.

[44] J. R. Mattox et al., Astrophys. J. 461, 396 (1996).

[45] G. Punzi, arXiv:physics/0401045. 